\title{
Neuroligin 2 Induced Allosteric Transition of Collybistin Underlies Inhibitory Postsynaptic Differentiation
}

\author{
PhD Thesis \\ in partial fulfilment of the requirements \\ for the degree "Doctor of Philosophy (PhD)" \\ in the Molecular Biology Program \\ at the Georg August University Göttingen, \\ Faculty of Biology
}

submitted by

Tolga Soykan

born in

Izmit 


\section{Declaration}

This thesis has been written independently and with no other sources and aids than quoted.

Tolga Soykan

Göttingen, March 31 ${ }^{\text {st }}, 2011$ 


\section{List of Publications}

Poulopoulos, A., Aramuni, G., Meyer, G., Soykan, T., Hoon, M., Papadopoulos, T., Zhang, M., Paarmann, I., Fuchs, C., Harvey, K., Jedlicka, P., Schwarzacher, S.W., Betz, H., Harvey, R.J., Brose, N., Zhang, W. \& Varoqueaux, F. (2009). Neuroligin 2 drives postsynaptic assembly at perisomatic inhibitory synapses through Gephyrin and Collybistin. Neuron $63,628-642$.

Hoon, M., Soykan, T., Falkenburger, B., Hammer, M., Patrizi, A., Schmidt, K.-F., Sassoè-Pognetto, M., Löwel, S., Moser, T., Taschenberger, H., Brose, N. \& Varoqueaux, F. (2011). Neuroligin-4 is localized to glycinergic postsynapses and regulates inhibition in the retina. Proc Natl Acad Sci USA. 108, 3053-8

Papadopoulos, T., Soykan, T. (2011). The role of Collybistin in Gephyrin clustering at inhibitory synapses: facts and open questions. Submitted for publication. 


\section{Table of Contents}

$\begin{array}{lr}\text { Acknowledgements } & 8\end{array}$

$\begin{array}{ll}\text { Abstract } & 10\end{array}$

$\begin{array}{ll}\text { List of Figures } & 11\end{array}$

$\begin{array}{ll}\text { 1.INTRODUCTION } & 12\end{array}$

$\begin{array}{ll}\text { 1.1. The Synapse } & 12\end{array}$

1.1.1. Synaptic transmission 12

1.1.2. The presynaptic active zone

$\begin{array}{ll}\text { 1.1.3. The postsynaptic density } & 16\end{array}$

$\begin{array}{ll}\text { 1.2. Synaptogenesis } & 18\end{array}$

1.2.1. The neuroligin-neurexin transsynaptic cell adhesion system 21

1.2.2. Other synaptic cell adhesion systems with synaptogenic roles 23

1.3. Synaptic inhibition 24

1.3.1. Glycine receptors

$\begin{array}{ll}\text { 1.3.2. GABA receptors } & 27\end{array}$

1.3.3. Comparison of synaptic clustering of glycine and $\mathrm{GABA}_{\mathrm{A}}$ receptors 30

1.4. Assembly of the inhibitory postsynapse 31

1.4.1. The Gephyrin scaffold 31

1.4.2. Collybistin, an adaptor for the Gephyrin scaffold 33

1.4.3. NL2, a neuronal factor that activates Collybistin 35

1.4.4. Putative roles of NL3 and NL4 at inhibitory synapses 38

$\begin{array}{ll}\text { 1.5. Aim of the study } & 39\end{array}$

2. MATERIALS \& METHODS 40

$\begin{array}{ll}\text { 2.1 DNA Constructs } & 40\end{array}$

2.1.1. Yeast-two-hybrid plasmids $\quad 40$

2.1.2. Expression plasmids 40

2.2 Antibodies and Labeling Reagents $\quad 42$

2.2.1. List of primary antibodies and working dilutions 42

2.2.2 Conjugated antibodies and reagents 43

2.3 Yeast two hybrid assays 43

2.4 Cell culture and transfection $\quad 44$

2.4.1. Cell-line culture and transfection 44

2.4.2. Primary neuron culture and transfection 44 
2.5.1. GST-fusion proteins $\quad 45$

2.6. Protein-Lipid binding assays 46

2.7. Crosslinking and identification of protein complexes 47

2.7.1. Preparation of crosslinked brainstem and spinal cord material 47

2.7.2. Immunoprecipitation of crosslinked complexes 47

2.8. Immunolabeling of transfected neurons and cell lines 48

2.8.1. Immunolabeling of transfected cell lines 48

2.8.2. Immunolabeling of transfected neurons 49

$\begin{array}{ll}2.9 \text { Statistical analysis } & 49\end{array}$

$\begin{array}{ll}\text { 3. RESULTS } & 50\end{array}$

3.1 Investigation of the roles of NL2 and NL4 at inhibitory synapses 50

3.1.1. NL2 and NL4 specifically interacts with the SH3 domain of Collybistin 50

3.1.2. NL4, like NL2, activates Collybistin and facilitates membrane 52 recruitment of Gephyrin

3.1.3. The NL2-Collybistin interaction involves the proline rich stretch of 53 NL2 and the SH3 domain of Collybistin

3.1.4. Both NL2 and NL4 are capable of establishing high order assemblies 56 with Gephyrin and Collybistin in vivo

3.1.5. Comparative analysis of $\mathrm{SH} 3$ domains reveals a putative 58

NL2 binding site on Collybistin

3.1.6. The $\mathrm{SH} 3$ domain of Collybistin may function as an autoinhibitory module 60

3.1.7. Interfering with the putative intramolecular interaction renders 63

Collybistin constitutively active

3.1.8. NL2-Collybistin interaction is necessary for NL2-mediated Collybistin activation 66

3.1.9. An intact PH domain is necessary for NL2-induced membrane 71

targeting of Collybistin

3.1.10. The SH3 domain inhibits binding of Collybistin to PI3P 72

3.1.11. NL2 leads to enhanced PI3P binding of Collybistin 76

3.1.12. Expression of exogenous $\mathrm{CB}_{\mathrm{SH}_{3+}}$ in Collybistin $\mathrm{KO}$ neurons 79 rescues Gephyrin clustering

3.1.13. Gephyrin-Collybistin interaction revisited: Gephyrin binds to $\mathrm{CB}_{\mathrm{SH}_{+}}$ on multiple sites

3.2 Investigation of the effects of serine phosphorylation of neuroligins on the Gephyrin binding motif 
4.1. NL2 as a functional organizer of inhibitory synapse assembly 87

4.1.1. Collybistin is a target of NL2 mediated signaling 88

4.1.2 SH3 domain-mediated autoinhibiton of Collybistin 90

4.1.3. PH domain-mediated membrane tethering of Collybistin 94

4.1.4. The putative catalytic role of the $\mathrm{DH}$ domain 99

4.1.5. Completing the puzzle: The tripartite complex of NL2-Gephyrin-Collybistin 100

4.1.5.1. NL2 - Gephyrin interaction 101

4.1.5.2. NL2 - Collybistin interaction 101

$\begin{array}{ll}\text { 4.1.5.3. Gephyrin-Collybistin interaction } & 101\end{array}$

4.1.6. A mechanistic model underlying the assembly of perisomatic inhibitory synapses 104

4.1.7. The role of NL4 at the inhibitory synapses 108

4.2. Regulated Association of Neuroligins with Scaffolding Molecules 110

\begin{tabular}{l} 
5. SUMMARY and CONCLUSIONS \\
\hline
\end{tabular}

$\begin{array}{lr}\text { 6. BIBLIOGRAPHY } & 114\end{array}$

$\begin{array}{lr}\text { Curriculum Vitae } & 129\end{array}$ 
Dedicated to my family 


\section{Acknowledgements}

This dissertation would not have been possible without the guidance and the support of several individuals who in one way or another contributed and extended their valuable assistance during the preparation of this study.

First and foremost I would like express my utmost gratitude to Dr. Alexandros Poulopoulos, not only for his guidance, incredible enthusiasm, continuous support and somewhat 'magical' inspiration during my thesis, but also for becoming one of my greatest friends. I wish to express my warm and sincere thanks to Dr. Frédérique Varoqueaux for her guidance and support, which helped me a lot to stay in the right track and to efficiently carry out my research. I am grateful to Prof. Nils Brose, head of my thesis committee, who provided critical input for my research, consistently displayed his faith in me and became a role model for scientific conduct and mentorship. I thank Prof. Andreas Wodarz and Dr. Dieter Klopfenstein who, as members of my thesis committee, provided valuable input and reflections on the progress of my work.

Many of my colleagues in the Department of Molecular Neurobiology of the Max Planck Institute of Experimental Medicine had significant contributions to this study. I had the privilege to become bench-mates with Dr.Theofilos Papadopoulos with whom I spent countless hours discussing the latest developments in the field and organizing the next experiment. His critical input into my research significantly widened my perspective. I would like to thank Klaus Hellmann for his very strong technical support. I would love to express my gratitude to my colleagues and lab-mates Noa Lipstein, Dr. Kalina Dimova, Dr. Benjamin Cooper, Dr. Mrinalini Hoon, Dr. Christoph Biesemann, Dr. Marilyn Tirard, Dr. Dilja Krüger, Dr. Liam Tuffy, Matthieu Hammer, Cordelia Imig, Ramya Nair, Christoph Bredack, Mika Kishimoto-Suga, Michiko Takeda, Burcu Kasapoglu (from the Department of Neurogenetics) and all the other members of the Department of Molecular Neurobiology who were all very friendly and supportive. I had the opportunity to work with an exceptional student, Helena Magliarelli. I'm grateful for her interest in my research and her ambition to contribute to my study.

The International Molecular Biology Graduate Program provided the ideal framework for academic development. I am grateful to Dr.Steffen Burkhardt, Ivana Bacakova and Kerstin Grüniger who provided amazing support throughout the course of study and beyond. I thank all my classmates from the Molecular Biology Program for all the fun we had together, particularly to Peer Hoopman, Katharina Hoppe, Seong Joo Koo, Christian Olendrowitz and Lope Florez Weidinger -the latter being also my flat mate for over three years.

I thank all my external collaborators for graciously providing reagents. I present my gratitude to Dr. Dirk Fasshauer and Esra Demircioglu, who shared their valuable expertise on various topics related to my research. I am also grateful to 
Prof. Hermann Schindelin and Bodo Sander for providing essential input for my research and also made me feel at home during my stay in Schindelin Lab in Würzburg. I would like to thank Prof. Heinrich Betz for showing deep interest in my progress and providing essential input that has helped me a lot in the course of my research.

I would like to express my gratitude to a few people who made the time I spent in Göttingen very special. I am very lucky to have met Anna Suska, with whom I spent two fantastic years. My life would have been much harder without her care and affection. Besides, the Göttingen experience would not have been so much fulfilling, had I not met Önder Intizamoglu.

I am grateful to those in my undergraduate years at Sabanci University, who had strong impact on my academic development. I owe my sincere gratitude to Prof. Zehra Sayers and Prof. Ugur Sezerman who were the first ones to guide my first steps towards my scientific career. I would like to thank my classmates and roommates from the university with whom I spent four fantastic years: Sedef Iskit, Canan Karakaya, Efnan Olcay, Feyza Tuncer, Azime Akman, Pinar Ayata, Berke Cetinöneri, Tuna Nisli, Emre Büyükcerci and many more. I am thankful to Prof. Fitnat Yildiz for hosting me as an internship student at UCSC where I had the opportunity to practice high-quality research in a very stimulating and friendly environment.

I feel urged to recognize the great importance of many elementary and high school teachers, most notable of which Semsa Uygun, who has been a precious friend and a life coach. I am grateful to Ismail Alpaslan and Bürgehan Hürcan who have been my best friends since earlier phases of my life.

Most of all, I am indebted to my family to whom I dedicate this work. I am particularly grateful to my beautiful mom Güler Soykan, my super dad Idris Soykan and my loving brother Arda Soykan, for their support and love. 


\section{Abstract}

Synaptic inhibition is vital to brain function. GABAergic and glycinergic synapses, mediating fast inhibition in the mammalian central nervous system (CNS), balance the activity of neural circuits. At the inhibitory postsynapse, GABA and glycine receptors accumulate around a scaffold composed of the protein Gephyrin, which is recruited to sites apposed to GABA or glycine releasing terminals through interaction with Neuroligin 2 (NL2), a member of the Neuroligin family of synaptic adhesion molecules that function as powerful synapse organizers. Another Gephyrin-binding molecule, Collybistin, regulates this recruitment process, functioning as a switch that is activated at sites of NL2 accumulation, leading to tethering of Gephyrin scaffold to the postsynaptic membrane and allowing the subsequent clustering of receptors at sites of transmitter release. Despite the well-documented studies about the critical role of Collybistin in the development of synaptic inhibition, the mechanism of Collybistin function has not yet been clearly identified. In the present study, a structure-function analysis on Collybistin, which was followed by protein-lipid interaction assays, led to the identification of two crucial molecular mechanisms: a conformational switch in Collybistin induced by NL2 binding and subsequent membrane tethering of Gephyrin-Collybistin complex via protein-lipid interactions. These data allow formulation of a coherent molecular model of the assembly of inhibitory synapses and covers a gap in our understanding of the development of synaptic inhibition in the mammalian CNS. 


\section{List of Figures}

Schema 1: Multiple adhesion molecules coexist at glutamatergic synapses 20

Schema 2: The domain structure and the splice variants of Collybistin 34

Schema 3: Current assembly model of inhibitory postsynapses 37

Figure 1: NL2 and NL4 interact with Collybistin 51

Figure 2: $\mathrm{NL} 4_{I C D}$, like NL2 ${ }_{\mathrm{ICD}}$, activates Collybistin and induces plasma membrane 52 recruitment of Gephyrin in transfected mammalian cells

Schema 4: Neuroligin domain structure.

Figure 3: Binding of Collybistin to NL2 and NL4 is mediated by a classical SH3-PXXP 55 mode of interaction

Figure 4: Both NL2 and NL4 assemble into higher order complexes with Gephyrin and $\quad 57$ Collybistin in vivo

Schema 5: Structure of the SH3 domain 59

$\begin{array}{ll}\text { Schema 6: The crystal structure of the autoinhibited Asef } & 61\end{array}$

Schema 7: Putative intra- and intermolecular contacts established by Collybistin 62 $\mathrm{SH} 3$ domain

Figure 5: The putative interface formed between $\mathrm{SH} 3$ and $\mathrm{DH} / \mathrm{PH}$ domains is $\quad$ 64-65 responsible for retaining Collybistin in an autoinhibited conformation

Figure 6: NL2-mediated activation of Collybistin depends on the NL2-Collybistin 67-68 interaction

Figure 7: The PH domain of Collybistin is necessary for membrane recruitment of Gephyrin

Figure 8: Collybistin specifically binds to Phosphatidylinositol-3-phosphate (PI3P) via its $\mathrm{PH}$ domain

Figure 9: NL2 enhances the lipid binding capacity of $\mathrm{CB} 2_{\mathrm{SH} 3+}$

Figure 10: Exogenous Collybistin rescues Gephyrin clustering upon transfection 77-78 to hippocampal neurons

Figure 11: Gephyrin binds to the linker region between $\mathrm{SH} 3$ and $\mathrm{DH}$ domains of Collybistin

Box 1: Neuroligin 3 is phosphorylated in vivo at Serine 799

Figure 12: Phosphorylation of Gephyrin-binding motif of NL3 might hinder its association with Gephyrin

Schema 8: Assembly model for the perisomatic inhibitory synapses 


\section{INTRODUCTION}

\subsection{The Synapse}

Nerve cells are capable of establishing rapid communication with each other over large distances with high precision. The sites of neuronal communication are called synapses. There are hundreds of billions of neurons in the human brain, and a single neuron forms and receives on average 1000 synaptic connections. Thus, a human brain can be considered as a large neuronal network, composed of at least $10^{14}$ synaptic connections (Murre et al., 1995). According to contemporary neuroscience, structural connectivity and the resulting network underlie all brain functions, from simple motor tasks to perception and cognition (reviewed by Sporns, 2002). Therefore, deciphering the mechanisms that underlie synaptic function is a crucial step in unraveling the complex functions of the brain.

\subsubsection{Synaptic transmission}

Neurons are electrically excitable cells. Via the action of membrane-embedded ion channels and pumps, resting neurons can maintain a difference in charge across their plasma membrane, which is known as the resting potential. Changes in this $-70 \mathrm{mV}$ voltage difference towards more positive or more negative values are called depolarization or hyperpolarization, respectively. If the plasma membrane of a neuron is depolarized sufficiently, typically to about $-55 \mathrm{mV}$, an action potential is initiated. Through the concerted action of sodium channels and positive feedback mechanisms, this depolarization is propagated throughout the neuron in the form of action potentials, causing it to "fire". Alternatively, brain stimuli may lead to hyperpolarization of the neuronal membrane, decreasing the probability of generating an action potential and causing the neuron to remain in a dormant state. This transition between resting and excited states, which is dictated by the external stimuli that a neuron 
receives, gives the neuron its capacity to encode information (reviewed by Gerstner et al., 1997).

A synapse is composed of a presynaptic axon terminal and of its juxtaposed region in a postsynaptic cell. Based on the structure of this apposition, synapses can be divided into two major categories: electrical and chemical synapses. At electrical synapses, an action potential generated in the presynaptic neuron flows directly into the postsynaptic cell through channels in specialized structures called gap junctions. The cytoplasmic coupling of neurons through electrical synapses results in rapid and synchronous firing of large groups of cells, which has evolutionary advantages for some organisms (reviewed by Bennett, 1997). However, due to the lack of heterogeneity in the types of responses evoked by electrical synaptic transmission, it is unlikely that these synapses account for the major form of the synaptic connectivity in the CNS of higher vertebrates.

In contrast, at chemical synapses, a change in membrane potential of the presynaptic neuron is capable of triggering a series of events that ultimately lead to the translation of this electrical signal into an extracellular chemical signal. Typically, during the firing of a presynaptic neuron, action protentials propagate along the axon leading to the opening of voltage-gated $\mathrm{Ca}^{2+}$ channels and to $\mathrm{Ca}^{2+}$-dependent release of chemical neurotransmitters from the nerve terminal into the synaptic cleft, a 20nm-wide gap between the pre- and postsynaptic neurons. Released transmitter molecules diffuse across the synaptic cleft and bind to ion channel-linked receptors embedded in the postsynaptic membrane. Binding leads to the opening of these channels, through which ions flow and thereby alter locally the membrane potential of the postsynaptic cell.

Through the diversity in the molecular composition of such neurotransmittergated receptors linked to ion channels, chemical synapses are capable of transmitting a great variety of signals, which may ultimately evoke excitatory or inhibitory (depolarizing or hyperpolarizing) responses in the receiving neuron. 
In contrast to electrical synapses, chemical synapses are also capable of producing long-lasting electrical changes in the postsynaptic cell. Therefore, due to inherent diversity of the duration, strength and nature of the responses elicited in the postsynaptic neuron, chemical synapses are the major contributors to the complexity of the brain functuion in higher vertebrates.

The effect of a chemical signal transmitted by a synapse -no matter whether it is excitatory or inhibitory- depends on whether the postsynaptic response is depolarizing or hyperpolarizing. This is determined by the type of ion channels stimulated by the neurotransmitter. Ion channel-linked receptors that allow for the influx of positively charged ions lead to depolarization of the postsynaptic membrane. This type of response is qualified as excitatory, and synapses that bear such cation-selective neurotransmitter receptor-channels are classified as excitatory synapses, since the signal mediated via these synapses typically increases the firing probability of the postsynaptic neuron. In the vertebrate CNS, synaptic excitation is generally mediated by the transmitter glutamate. On the other hand, transmission of a signal through synapses that have $\mathrm{Cl}^{-}$selective ligand-gated ion channels generally leads to hyperpolarization of the postsynaptic neuron. Since hyperpolarization hinders excitation, these synapses are classified as inhibitory. They are typically gated by the transmitters glycine and $\gamma$-aminobutyric acid (GABA) in the vertebrate brain.

Each neuron in the CNS receives a large number of synaptic inputs from other neurons. Depending on whether they are excitatory or inhibitory, these different inputs either reinforce or cancel each other out. These cooperative or competing inputs are integrated in the somatodendritic compartment of the receiving neuron. To initiate a new action potential, the postsynaptic neuron usually requires the input of multiple excitatory postsynaptic potentials (EPSPs), which lead to a significant postsynaptic depolarization. When a postsynaptic cell receives multiple excitatory inputs simultaneously (spatial summation), or successive EPSPs are generated at the same synapse in a short time interval (temporal summation), cumulated EPSPs can reach the threshold for necessary 
to trigger an action potential (reviewed by Magee, 2000). In turn, inhibitory postsynaptic potentials (IPSPs), which are generated at inhibitory synapses, counteract EPSPs, shift the membrane potential further away from this threshold and reduce the excitability of the postsynaptic neuron. Thus, the counterbalancing effects of excitatory and inhibitory synapses determine the decision of a postsynaptic neuron to fire or not to fire at a given time.

\subsubsection{The presynaptic active zone}

At chemical synapses, the conversion of the electrical signal into a chemical signal takes place at active zones, which are protein-rich specialized compartments of the presynaptic nerve terminal. Active zones are sites where neurotransmitter-carrying synaptic vesicles cluster, tether and fuse to the plasma membrane to release their content into the synaptic cleft. Active zones are very closely and precisely apposed to postsynaptic densities (PSDs) where neurotransmitter receptors are clustered.

Besides being the site where synaptic vesicle fusion takes place, the plasma membrane at the active zone additionally accommodates voltage-gated calcium channels. Upon arrival of an action potential at a presynaptic nerve ending, voltage-dependent calcium channels allow for the influx of $\mathrm{Ca}^{2+}$ ions along a concentration gradient. This local increase in $\mathrm{Ca}^{2+}$ concentration very rapidly triggers the exocytosis of synaptic vesicles. Thus, via the action of voltage-gated $\mathrm{Ca}^{2+}$ channels, the excitation of a neuron is coupled to the secretion of neurotransmitter from its presynaptic active zone.

The coupling between excitation and the sudden release of synaptic vesicles is a tightly regulated process that is dependent on cytosolic $\mathrm{Ca}^{2+}$ concentration. This process is spatially confined to active zones and driven by an array of molecules which regulate the positioning of synaptic vesicles at the active zones and mediate membrane fusion events (reviewed by Wojcik \& Brose, 2007 and Rizo \& Rosenmund, 2008). The core fusion machinery is composed of synaptic 
SNARE proteins located on the presynaptic and vesicular membranes, which are capable of adjusting a metastable configuration through the action of regulatory proteins, such as complexins. Other active zone proteins, including Munc13 and RIM $\alpha$, facilitate SNARE complex assembly and thereby enabling the positioning of fusion competent vesicles at the active zones. Finally, a $\mathrm{Ca}^{2+} /$ phospholipid binding sensor protein Synaptotagmin, intreracts with the SNARE complex and via the action of its lipid binding domains, it drives the fusion of the vesicular and the plasma membrane in response to $\mathrm{Ca}^{2+}$.

The cytoskeletal matrix at the active zone is termed cytomatrix and is rich in a variety of proteins with roles in vesicle fusion. Apart from the regulatory proteins discussed above which constitute the release machinery, and the cytoskeletal proteins, which constitute the frameork of the active zone, the cytomatrix accommodates anchoring and scaffolding molecules, such as CASK, Bassoon, Piccolo, which link the cytoskeletal elements to ion channels and fusion machinery, thus ensuring the proper assembly of the active zone (reviewed by Schoch \& Gundelfinger, 2006). This diverse set of active zone-specific proteins, along with presynaptic cell adhesion molecules, such as cadherins, protocadherins, NCAM, SynCAM, Netrins, Neurexins (reviewed by Yamagata et al., 2003) which are responsible for the assembly of the active zone and its proper alignment with the PSD via homophilic or heterophilic interactions, act in a concerted manner to determine synapse features and the signal that is transmitted to the postsynaptic cell.

\subsubsection{The postsynaptic density}

Neurotransmitter molecules released in the synaptic cleft bind to ligand-gated ion channels embedded in the postsynaptic membrane. The interactions between the neurotransmitters and the extracellular surface of the receptor molecules lead to allosteric changes in the channel structure, resulting in opening of hydrophilic pores through which ions traverse the plasma membrane. This gating mechanism of ion channel-linked receptors allows them 
to respond to neurotransmitters by changing the conductance of the postsynaptic membrane. Depending on the biochemical characteristics of their pore structures, these receptors are selectively permeable to specific positively or negatively charged ions. Receptors that allow $\mathrm{Na}^{+}$influx, such as glutamate receptors, contribute to a postsynaptic depolarization, which then plays a part in generating an action potential in the postsynaptic cell. The activation of $\mathrm{Cl}^{-}$ permeable receptor channels, on the other hand, such as GABA and glycine receptors, results in hyperpolarization of the membrane potential, shunting current and suppressing excitation in the postsynaptic neuron. Thus, these neurotransmitter receptors are responsible for the transduction of the incoming presynaptic inputs.

Synaptic transmission is rapid and precise, and neurotransmitter receptors are densely clustered at the membrane facing presynaptic release sites. They are part of a specialized electron-dense, protein-rich apparatus termed the postsynaptic density (PSD). At the PSD a large number of receptors, cell adhesion molecules, scaffolds, cytoskeletal elements and signaling molecules assemble to form a large functional entity that ensures efficient synaptic transmission (reviewed by Triller \& Choquet, 2003). The high-density clustering of receptor molecules positioned in close proximity to release sites facilitates synaptic transmission by limiting the time required for neurotransmitter molecules to reach their cognate receptors.

Selective neurotransmitter binding and differential permeability to anions and cations make neurotransmitter receptors the primary determinants of the characteristics of the postsynaptic response. Nevertheless, other constituents of the PSD also have important structural and functional features that allow them to regulate the transmission properties of a synapse. For instance, through their cytoplasmic domains, the postsynaptic components of transsynaptic cell adhesion complexes, such as Neuroligins (Scheiffele et al., 2000; Graf et al., 2004), SynCAM (Biederer et al., 2002), Netrin-G ligands (NGL) (Kim et al., 2006) and LRRTMs (Linhoff et al., 2009), recruit further proteins to the PSD with 
specific signaling and scaffolding roles. Such scaffolding molecules harbor multiple protein-protein interaction modules and can oligomerize, and thereby link neurotransmitter receptors with the other constituents of the PSD to maintain structural coherence. For example, PSD-95 (postsynaptic density protein of $95 \mathrm{kDa}$ ), an excitatory synapse-specific scaffolding molecule, bridges NMDA (N-methyl-D-aspartate) receptors (Kornau et al., 1995) and TARP [transmembrane AMPA ( $\alpha$-amino-3-hydroxy-5-methylisoxazole-4-propionic acid) receptor regulatory protein]-associated AMPA receptors (Chen et al., 2000) with the other constituents of the PSD. Similarly Gephyrin, a major scaffolding protein found at GABAergic and glycinergic inhibitory synapses, interacts with

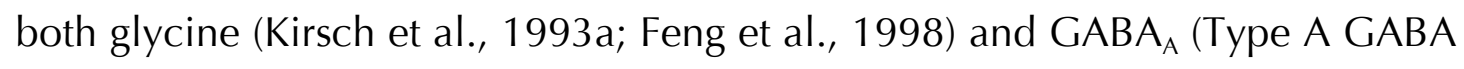
$(\gamma$-aminobutyric acid)) receptors (Essrich et al., 1998; Sassoè-Pognetto et al., 2000; Studler et al., 2002; § 1.3.1\& 1.3.2). Therefore, it appears that, the whole ensemble of receptors, scaffolds, cytoskeletal elements, cell adhesion molecules and other signaling molecules act in a concerted manner to drive and maintain the proper assembly and function of postsynaptic specializations. The differential trafficking, distribution, posttranslational modification and allosteric regulation of receptors and receptor-associated molecules may underlie the specific differentiation of postsynapses.

\subsection{Synaptogenesis}

The formation of synapses, termed synaptogenesis, typically involves a physical contact between an axonal growth-cone filopodium and a dendritic shaft or filopodium. Guided by their growth cones, growing axons often traverse considerable distances until they reach their target regions where they are destined to establish a contact. During this process of pathfinding, termed axon guidance, the growth cone constantly interacts with its surroundings and "decides" on which route to take based on repulsive and attractive signals presented by extracellular cues and intercellular contacts. The main set of 
proteins involved in axon guidance includes Ephrins and Eph receptors, Netrins, Plexins and Semaphorins (reviewed by Chilton, 2006).

Once a growing axon reaches its target area, axons -and sometimes also dendrites- extend filopodia that make initial axodendritic contacts. These transient contacts constitute an important step in target recognition as the axon selects individual target cells from a large number of candidates. Cadherins and protocadherins (reviewed by Takeichi, 2007), two very prominent protein families involved in cell adhesion, have been suggested to mediate these initial axodendritic contacts that underlie target recognition.

After target recognition, these initial axodendritic contacts that endure a stringent selection procedure and undergo spatially and temporally controlled changes in morphology and molecular content to form mature synapses. These are characterized by the specific accumulation of synaptic vesicles and active zone components within the (presynaptic) axon, in close apposition to a (dendritic) postsynaptic membrane, containing a super-complex of neurotransmitter receptors, scaffold proteins and intracellular signal transduction molecules. The process of synaptic differentiation is an essential step in synaptogenesis. In the course of these reciprocal changes in the nascent pre- and postsynaptic membranes, a crosstalk between these two compartments ensures that coordinated differentiation takes place and compatible release and receptor machineries are coupled to each other. Not surprisingly, transsynaptic signaling mediated by cell adhesion molecules has been identified to drive this coordinated assembly of pre- and postsynaptic specializations (reviewed by Craig, Graf \& Linhoff, 2006).

During the past 10 years, research focusing on synaptic cell adhesion has proven that transsynaptic cell adhesion systems are not only required for maintaining a physically strong synaptic contact, but also have essential roles in synaptogenesis. The major evidence for the critical role of cell adhesion molecules were obtained from a coculture assay established by Scheiffele et al., 
(2000); an in vitro system where neurons were cultured with non-neuronal cells heterologously expressing candidate adhesion molecules on their surfaces. In this assay, each candidate molecule was assessed for its potential roles in contact formation and inducing pre- and postsynaptic differentiation of axonal and dendritic contacts on neurons. In this study and in other subsequent studies, a handful of cell adhesion molecules were identified to promote pre- and postsynaptic differentiation, including Neuroligin 1, Neuroligin 2 (Scheiffele et al., 2000), SynCAM1 (Biederer et al., 2002), Neurexin $1 \beta$ (Graf et al., 2004), Neurexin $1 \alpha$ (Kang et al., 2008), EphB and EphrinBs (Aoto et al., 2007; Kayser et al., 2006), Netrin-G ligand (Kim et al., 2006) and LRRTM1 (Linhoff et al., 2009) (Schema 1).

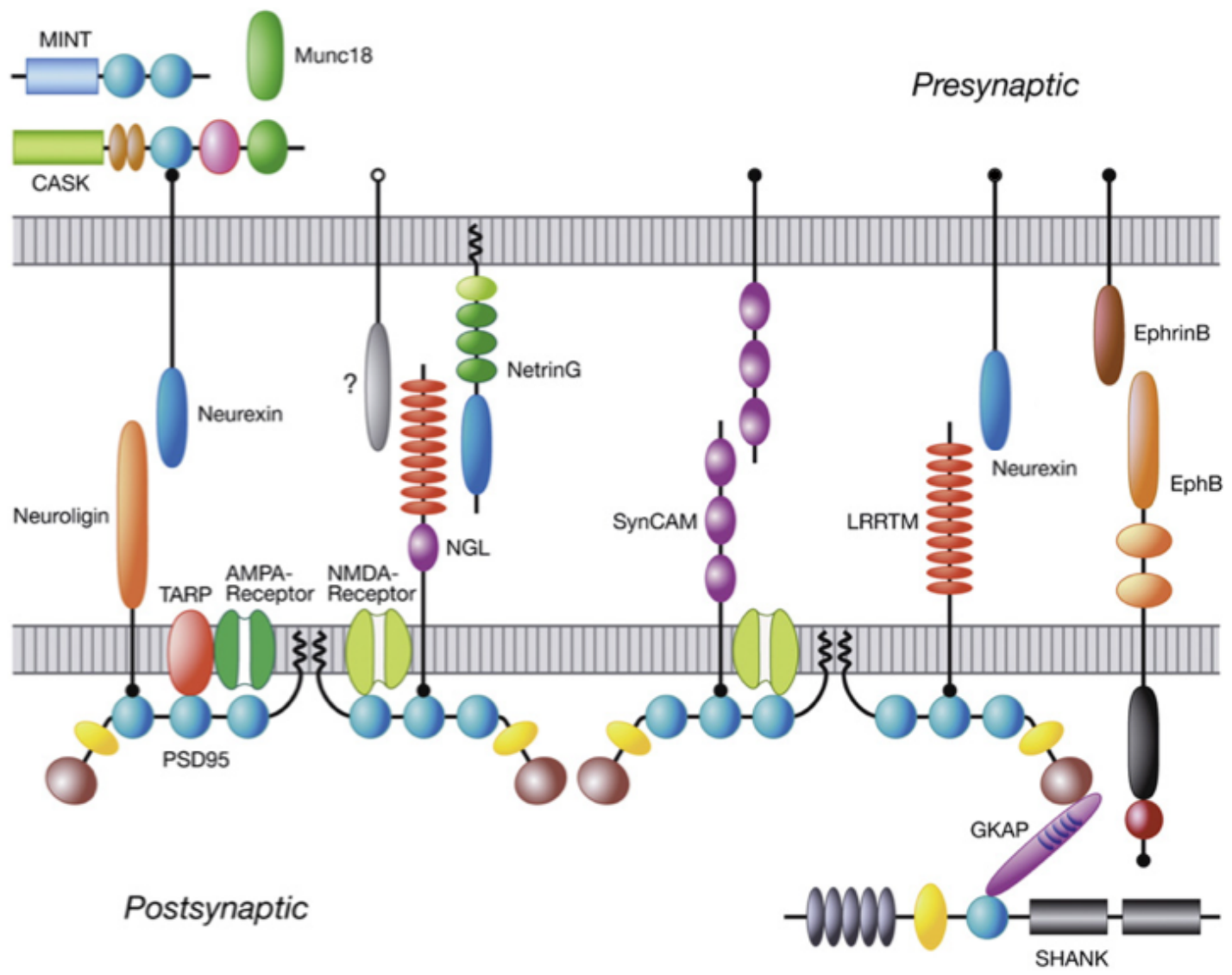

Schema 1: Multiple adhesion molecules coexist at glutamatergic synapses: The image displays transsynaptic cell adhesion complexes that exhibit synaptogenic activity, identified via neuron-fibroblast coculture assay established by Scheiffele et al., (2000), including heterophilic Neuroligin-Neurexin, Netrin-G Ligand-Netrin-G, LRRTM-Neurexin, EphBEphrinB complexes and homophilic SynCAM complex. Among the cell adhesion systems identified so far, only the Neuroligin-Neurexin complex appears to be functional in both excitatory glutamatergic and inhibitory GABAergic and glycinergic synapses. Figure modified after Brose, 2009. 


\subsubsection{The neuroligin-neurexin transsynaptic cell adhesion system}

Among the cell adhesion molecules implicated in synaptogenesis, the Neuroligin-Neurexin heterophilic adhesion system was the first one to be identified and is currently the best characterized complex. Presynaptic Neurexins pair with postsynaptic Neuroligins (NLs) and each signals via its partner to induce the recruitment of the respective synaptic machineries on the two synaptic membranes. Although they are dispensable for the apposition of pre- and postsynaptic specializations, mouse genetic studies have shown that both Neuroligins (Varoqueaux et al., 2006) and $\alpha$-Neurexins (Missler et al., 2003) are essential for proper synapse function. The dysfunction of Neuroligins and Neurexins leads to deficits in recruitment of the components of the pre- and postsynaptic apparatus.

In mammals, there are three neurexin genes, each of which has both an upstream promoter that drives the expression of the larger $\alpha$-Neurexins, and a downstream promoter that generates the short $\beta$-Neurexins (Tabuchi \& Südhof, 2002). N- and O-glycosylation and alternative splicing confer additional diversity to Neurexins. $\beta$-Neurexins contain a single LNS domain (laminin, neurexin, sex-hormone-binding protein domain), while $\alpha$-Neurexins contain six LNS domains organized into modules with three EGF-like domains. Neurexins can coordinate a $\mathrm{Ca}^{2+}$ ion with their extracellular domains and they associate with NLs in a $\mathrm{Ca}^{2+}$-dependent manner (Nguyen \& Sudhof, 1997).

In rodents, four paralogous Neuroligin (NL) genes encode the type-I transmembrane proteins NL1 (Ichtchenko et al., 1995), NL2, NL3 (Ichtchenko et al., 1996) and NL4 (Jamain et al., 2008). The extracellular domains of NLs are highly conserved among the four paralogues. This domain harbors a Neurexin binding site and is homologous to acetylcholinesterase (AChE). The cytoplasmic domains of NLs are more divergent in sequence, although they all share a Cterminal PDZ-binding motif with which they associate to PDZ domaincontaining molecules, as well as a highly conserved Gephyrin binding motif. 
Via these protein-protein interaction motifs, NLs are capable of associating with scaffolding molecules localized at excitatory (PSD-95; Irie et al., 1997), inhibitory (Gephyrin; Poulopoulos et al., 2009) and both types of synapses (SSCAM; lida et al., 2004).

Despite their extensive similarity and shared motifs for binding to postsynaptic scaffolds, Neuroligin paralogues appear to have differences in function and localization in vivo. NL1 is localized mainly to excitatory synapses (Song et al., 1999) and NL1 KO mice show deficits in NMDA receptor transmission, while inhibitory transmission is unaffected (Chubykin et al., 2007). On the other hand, NL2 localizes exclusively to inhibitory synapses (Varoqueaux et al., 2004) and NL2 KO mice exhibit reduced inhibitory transmission in the somatosensory cortex (Chubykin et al., 2007) and a reduction of GABAergic transmission in the hippocampus and deficits in both GABAergic and glycinergic transmission in the brainstem (Poulopoulos et al., 2009). In contrast to the restricted distribution of NL1 and NL2, NL3 and NL4 were shown to be shared components of both types of synapses. In dissociated hippocampal cultures, NL3 was shown to localize to both GABAergic and glutamatergic synapses and coaggregated with both excitatory and inhibitory scaffolding molecules (Budreck and Scheiffele, 2007). In a recent study, which addressed the localization of NL4 in different parts of the CNS, it was shown that NL4 is targeted to glycinergic postsynapses in various brain regions including the retina, brainstem, spinal cord and to Gephyrin-positive inhibitory synapses in the thalamus and the colliculi (Hoon et al., 2011). However there is also growing evidence that NL4 might be associated with excitatory synapses in other parts of the brain (Matthieu Hammer, Göttingen; personal communication). These observations have led to an hypothesis, according to which these Neuroligin paralogues are responsible for establishing the balance between excitation and inhibition in the CNS (Prange et al., 2004; Cline, 2005; Levinson \& El-Husseini, 2005).

The mechanisms underlying the specific localization of Neuroligins at different typses of synapses are still largely elusive. Studies addressing the excitatory 
versus inhibitory synapse selectivity of Neuroligins demonstrated that Neuroligin localization is determined by the presynaptic component, and that alternative splicing of Neuroligins and Neurexins drives this process. Analysis of NL1 splice variants suggested that a splice site B in the NL1 extracellular domain contains an $\mathrm{N}$-linked glycosylation site that determines specificity for presynaptic Neurexins. B-site-containing NL1 was shown to interact specifically with $\beta$-Neurexins lacking splice insert 4, while NL2 preferentially associates with $\alpha$-Neurexins and variants of $\beta$-Neurexins lacking the splice insert 4 . (Boucard et al., 2005; Chih et al., 2006; Graf et al., 2006; Kang et al., 2008). However, these findings have been functionally disputed (Chubykin et al. 2007). Furthermore an extensive analysis of $\beta$-Neurexin-Neuroligin interactions revealed that the effect of alternative splicing on determining the strength of $\beta$ Neurexin/Neuroligin association is rather limited. In contrast to previously published results, all NLs were found to bind all $\beta$-Neurexins, irrespective of the splice isoform of either partner (Koehnke et al., 2010). These finding indicate that there is no evidence for a "binary code" for Neuroligin-Neurexin cell adhesion systems, which would require that some pairs of NLs and $\beta$-Neurexins fail to bind to each other. It rather seems that multiple adhesion systems act in synergy to convey a more complex code that would lead to cell-type-or synapse-specific synaptogenesis. Clearly, further research is required to

understand how specific Neuroligin and Neurexin pairs are capable of localizing to and driving the assembly of excitatory or inhibitory pre- and postsynapses.

\subsubsection{Other synaptic cell adhesion systems with synaptogenic roles}

In addition to Neuroligin-Neuerxin complex, SynCAM is a prominent member of the group of cell adhesion molecules implicated in synaptogenesis. SynCAM is a homophilic adhesion molecule localized on both sides of the synapse. Heterologous expression of SynCAM in non-neuronal cells leads to excitatory presynapse differentiation in hippocampal neurons (Biederer et al., 2002). Another recently identified molecule to have roles in synaptogenesis are 
Leucine-Rich Repeat Neuronal Transmembrane Proteins (LRRTMs). Like Neuroligins, LRRTMs are located in the postsynaptic membrane, and form transsynaptic complexes with $\beta$-Neurexins and induce glutamatergic presynaptic differentiation (Linhoff et al., 2009; de Wit et al., 2009; Ko et al., 2009; Siddiqui et al., 2010). Yet another adhesion system implicated in synaptogenesis involves the presynaptic EphrinB and postsynaptic EphB. EphB directs synaptogenesis by modulating filopodia motility. Upon stable binding of axonal EphrinB, EphB is able to mediate NMDA and AMPA receptor clustering and formation of dendritic spines (Kayser et al., 2008).

The multiplicity of cell adhesion systems with similar tasks in synaptogenesis gives rise to overlaps and redundancies in their functions, as illustrated by only moderate phenotypic changes observed in Neuroligin knockouts (Varoqueaux et al., 2006; Chubykin et al., 2007). The members of different cell adhesion complexes, or even the paralogues or splice isoforms of the same cell adhesion proteins may exist together in the same synapse, binding to the same scaffolding molecules and performing the same function. However, the complexity of information processing in the CNS and the variations in the types of synaptic contacts throughout the brain indicate that these multiple cell adhesion systems might in fact be necessary to achieve specificity at each synapse. The combinations of multiple transsynaptic cell adhesion complexes might function synergistically to drive specific differentiation of synapses deployed with the constituents of a specific transmission system (reviewed by Brose, 2009). Among the cell adhesion systems identified until now, only the Neuroligin-Neurexin complex appears to be functional in both excitatory glutamatergic and inhibitory GABAergic and glycinergic synapses. In fact, the Neuroligin-Neurexin complex appears to be the only well-established adhesion system at inhibitory synapses. 


\subsection{Synaptic inhibition}

In the CNS, almost all functions, including cognition, decision-making, sleep and motor control rely on a precise balancing action of synaptic inhibition on excitation. The critical role of synaptic inhibition is also apparent in disease, such as epilepsy, where impairments in inhibitory synaptic transmission lead to abnormal osciallatory activity in the brain (Brenner, 2004). Many of the diseases of the CNS, including Parkinson's disease, autism, Tourette's syndrome and schizophrenia may be caused by deficits in the balance between excitation and inhibition. Therefore, it is critical to understand the architecture of inhibitory synapses and mechanisms underlying inhibitory synapse assembly.

GABA and glycine are the two major neurotransmitters that mediate synaptic inhibition in the CNS. GABAergic inhibition dominates in the forebrain while glycinergic transmission is more prominent in the spinal cord and the brainstem. Despite these regional differences GABA and glycine mediated inhibition processes are very similar. The fact that the two transmitters systems share the same vesicular transporter (Gasnier, 2000; Wojcik et al., 2006) and the same postsynaptic scaffolding molecule (Gephyrin, § 1.4.2), and the presence of $\mathrm{GABA}$ and glycine co-releasing terminals with mixed postsynaptic sites indicate that the two neurotransmitter systems may cooperate in determining the strength of inhibition.

\subsubsection{Glycine receptors}

Glycine receptors are $\mathrm{Cl}^{-}$selective ionotropic receptors belonging to the nicotinic receptor superfamily, which also includes $G_{A B A^{\prime}}$ nicotinic acetylcholine, and serotonin type 3 receptors, all mediating fast synaptic transmission in the CNS. Like the other members of this group of receptors, GlyRs are heteropentameric oligomers (Langosch et al., 1988). A typical GlyR is composed of combinations of five membrane spanning proteins from two major 
classes, $\alpha$, with four possible subunits, and $\beta$, with a single subunit. The glycine binding pocket is formed at the interface between the $\alpha$ and $\beta$ subunits in the assembled pentameric receptor. Ligand binding causes these receptors to open their pores and allow for $\mathrm{Cl}^{-}$flux. Detailed analysis of glycine receptor subtypes in the CNS indicated that most glycine receptors are composed of $\alpha$ and $\beta$ subunits with a stoichiometry of $\alpha 2: \beta 3$ (Grudzinska et al., 2005).

Strychnine, a toxin that causes intense muscle contractions, potently blocks the inhibitory actions of glycine. Strychnine poisoning leads to hyperexcitability of motorneurons resulting in convulsions and respiratory failure. In addition, it produces strong pain syndromes and hyperacuity of visual and auditory responses via disinhibition of sensory processing areas, i.e. dorsal horn of the spinal cord, cochlear nucleus, inferior colliculus and retina. Due to its high affinity binding to endogenous GlyRs, radiolabeled strychnine was used for characterizing GlyR distribution in the CNS (Probst et al., 1986). In parallel to the specific effects of strychinine poisoning, GlyRs were shown to be highly prevalent in spinal cord, where they are involved in motor reflex circuits and provide inhibitory synaptic inputs onto pain sensory neurons. Glycinergic inhibition is also abundant in the retina and various parts of the brainstem, such as the cochlear nucleus (reviewed by Lynch, 2004). Recently GlyRs were also shown to be localized to various parts of the forebrain, including the bstriatum and parts of the midbrain (Baer et al., 2009).

Synaptic clustering of GlyRs is mediated by the inhibitory synapse-specific scaffolding molecule Gephyrin, as illustrated by the severe deficits in postsynaptic GlyR clustering in its absence (Kirsch et al., 1993a; Feng et al., 1998). Gephyrin interacts specifically with the cytosolic loop of the GlyR $\beta$ subunit, (Meyer et al., 1995), the common and invariable component of most of the GlyRs in the CNS. An amphipathic helix within the intracellular domain of the $\beta$ subunit mediates the interaction with Gephyrin. Furthermore, incorporation of this helix into the $\beta 1$-subunit of the $\mathrm{GABA}_{\mathrm{A}}$ receptor, to the NMDA receptor NR1 subunit or to green fluorescent protein (GFP) induces 
binding of these proteins to Gephyrin (Meyer et al., 1995; Kins et al., 1999; Kneussel et al., 1999a). In heterologous cells, the GlyR $\beta$ subunit, but not $\alpha$ subunits, is recruited to intracellular Gephyrin aggregates. In neurons, laterally diffusing GlyRs can be captured and clustered at synaptic sites by Gephyrin (Meier et al., 2001). Being a molecule that can control the subcellular distribution of glycine receptors and with its presence at glycinergic postsynapses, Gephyrin is an important molecule for anchoring GlyRs to inhibitory postsynaptic sites.

\subsubsection{GABA receptors}

GABA receptors are a major class of neurotransmitter receptors that respond to GABA, the main inhibitory neurotransmitter in the CNS. The two main classes of GABA receptors are the ionotropic ligand gated $\mathrm{Cl}^{-}$channels termed $\mathrm{GABA}_{\mathrm{A}}$ and the $G$ protein-coupled metabotropic receptors called $G_{A B A}$. GABA receptors belong to the family of nicotinic receptors; typically composed of a pentameric assembly of different subunits. $\mathrm{GABA}_{\mathrm{A}}$ receptors mediate fast synaptic inhibition in the CNS. Apart from GABA, the active site of $\mathrm{GABA}_{\mathrm{A}}$ receptors is a binding site for several drugs, including bicuculline. Furthermore certain typses of $\mathrm{GABA}_{\mathrm{A}}$ receptors have allosteric binding sites for anxiolytic drugs such as benzodiazepines and barbiturates as well as neurosteroids and ethanol (reviewed by Bormann, 2001). Due to the specific effects of these chemicals, $\mathrm{GABA}_{\mathrm{A}}$ receptors have been a focus of intense study.

To date, several subunit isoforms for $\mathrm{GABA}_{\mathrm{A}}$ receptors have been identified, including six types of $\alpha$ subunits, three $\beta$, three $\gamma$ and $a \delta$, an $\varepsilon$, a $\pi$ and a $\theta$ subunit. The most commonly found $\mathrm{GABA}_{\mathrm{A}}$ receptors adopt a $2 \alpha: 2 \beta: 1 \gamma$ stoichiometry (Chang et al., 1996). The roles of $\delta, \varepsilon, \pi$ and $\theta$ subunits are still largely elusive. However it is likely that they substitute for the $\gamma$ subunit, forming alternative combinations with the $\alpha$ and $\beta$ subunits (reviewed by Sieghart et al., 1999). 
Although the exact subunit composition of any of the $\mathrm{GABA}_{\mathrm{A}}$ receptor subtypes in the CNS is still not identified, growing evidence points towards variations of their composition from region to region in the brain, or even between different parts of the same neuron. For example, immunocytochemistry and in situ hybridization studies revealed that $\alpha 1$ subunit-containing $\mathrm{GABA}_{\mathrm{A}}$ receptors are equally distributed to all inhibitory synapses on somatic and dendritic segments, while $\alpha 2$ subunit containing receptors are specifically localized to axo-axonic synapses on the axon initial segment (Nusser et al., 1996; Fritschy et al., 1998). In addition, it was demonstrated that $\mathrm{GABA}_{\mathrm{A}}$ receptors containing the $\alpha 6$ subunit is exclusively expressed in the cerebellum and the $\alpha 5$ subunit is specifically expressed in the hippocampus (Pirker et al., 2000). Furthermore, in the cerebellum, the $\gamma 2$ subunit-containing $\mathrm{GABA}_{\mathrm{A}}$ receptors were shown to be localized to synaptic sites whereas receptors that contain the $\delta$ subunit were targeted to extrasynaptic sites (Nusser et al., 1998). The significance of the compositional variety of $\mathrm{GABA}_{\mathrm{A}}$ receptors is still largely unknown, but it is possible that receptors with different combinations of subunits exhibit different functional properties, such as affinity to GABA, channel gating, kinetics and pharmacological characteristics. The subunit identity may also have implications for the transport and subcellular localization of the individual $\mathrm{GABA}_{\mathrm{A}}$ receptors.

One functional implication of subunit identity of $\mathrm{GABA}_{\mathrm{A}}$ receptors may involve the specific roles of different $\mathrm{GABA}_{\mathrm{A}}$ receptor subtypes in tonic and phasic inhibition. Tonic inhibition, mediated by extrasynaptic $\mathrm{GABA}_{\mathrm{A}}$ receptors, sets a baseline state of inhibition, which leads to steady-state hyperpolarization of a given neuron in the absence of synaptic activity. Deficits in tonic inhibition coinsides with increased epileptogenic activity, exemplifying the former's critical role in modulating neuronal excitability by setting a threshold for action potential generation. Phasic inhibition is mediated by synaptically clustered $\mathrm{GABA}_{\mathrm{A}}$ receptors and is correlated with synaptic activity. The differences between tonic and phasic inhibition may rely on the different subunit composition and localization of the associated $\mathrm{GABA}_{\mathrm{A}}$ receptors. Detailed 
morphological analysis revealed that the $\gamma 2$ subunit is essential for the synaptically clustered $\mathrm{GABA}_{\mathrm{A}}$ receptors, while the $\delta$ subunit containing $\mathrm{GABA}_{\mathrm{A}}$ receptors are exclusively extrasynaptic and required for mediating tonic inhibition. Furthermore, among the $\alpha$ subunits, $\alpha 1, \alpha 2$ and $\alpha 3$ tend to be involved in the assembly of synaptic $\mathrm{GABA}_{\mathrm{A}}$ receptors, while $\alpha 4, \alpha 5, \alpha 6$ subunit containing $\mathrm{GABA}_{\mathrm{A}}$ receptors are preferentially localized to extrasynaptic sites (reviewed by Lüscher \& Keller, 2004). Therefore in addition to regulating their functional features, the differential subunit composition may be involved in the mechanisms that determine the synapse type-specific targeting of $\mathrm{GABA}_{\mathrm{A}}$ receptors in neurons.

Similar to GlyRs, GABA $A_{A}$ receptors are also clustered via Gephyrin at inhibitory postsynapses, although compared to GlyR-Gephyrin association, the $G_{A B A_{A}} R-$ Gephyrin relationship is less well understood. In areas of the CNS where GlyRs are not abundant, Gephyrin colocalizes with postsynaptic $\mathrm{GABA}_{\mathrm{A}}$ receptors (Essrich et al., 1998; Sassoè-Pognetto et al., 2000; Studler et al., 2002). Antisense depletion of Gephyrin expression in cultured neurons and Gephyrin deletion in mice leads to reduced postsynaptic clustering of $\mathrm{GABA}_{\mathrm{A}}$ receptors containing the $\alpha 2$ and $\alpha 3$ subunits (Essrich et al., 1998; Kneussel et al., 1999b; Kneussel et al., 2001), indicating that, similar to its role in glyinergic synapses, Gephyrin is additionally responsible for $\mathrm{GABA}_{\mathrm{A}}$ receptor clustering. Consistent with this finding, it was recently demonstrated that the $\alpha 2$ and $\alpha 3$ subunits, but not the $\alpha 1$ subunit, of $\mathrm{GABA}_{\mathrm{A}}$ receptors are capable of directly interacting with Gephyrin (Tretter et al., 2008; Saiepour et al., 2010). Strikingly, in cultured hippocampal neurons and in spinal cord sections, loss of Gephyrin does not affect synaptic clustering of $\alpha 1$ subunit-containing $\mathrm{GABA}_{\mathrm{A}}$ receptors (Kneussel et al., 2001; Levi et al., 2004), although Gephyrin appears to cocluster with $\alpha 1$ containing $\mathrm{GABA}_{\mathrm{A}}$ receptors in vivo (Studler et al., 2002). It appears that at a subset of GABAergic postsynapses, particularly ones that contain the $\alpha 1$ subunit, the absence of Gephyrin can be compensated for by alternative mechanisms. Furthermore, the subunit identity of $\mathrm{GABA}_{\mathrm{A}}$ receptors seems to also regulate the postsynaptic clustering characteristics of these receptors. 


\subsubsection{Comparison of synaptic clustering of glycine and $G_{A B A_{A}}$ receptors}

Although Gephyrin appears to be a common node for clustering both $\mathrm{GABA}_{\mathrm{A}}$ receptors and GlyRs, there are major differences in the role of Gephyrin during the assembly of GABAergic or glycinergic postsynapses. Remarkably, loss of Gephyrin leads to complete loss of clustered glycine receptors, while only a fraction of $\mathrm{GABA}_{\mathrm{A}}$ receptors (the ones containing $\alpha 2$ subunits) are partially affected. Interestingly, while synaptic clustering of GlyRs depends entirely and strictly on Gephyrin, $\mathrm{GABA}_{\mathrm{A}}$ receptors are capable of forming synaptic clusters before being detectably colocalized with Gephyrin (Danglot et al., 2003), indicating that $\mathrm{GABA}_{\mathrm{A}}$ receptors can assemble into initial synaptic clusters independent of Gephyrin. These observations show that Gephyrin has distinct roles in clustering glycine and $\mathrm{GABA}_{\mathrm{A}}$ receptors. Gephyrin appears to be present at every step of GlyR clustering at inhibitory synapses, including the intracellular transport of individual GlyRs to synaptic sites (Hanus et al., 2004), and the stabilization of GlyRs once they are insterted in the plasma membrane. In contrast, Gephyrin is responsible only for stabilizing previously clustered $\mathrm{GABA}_{\mathrm{A}}$ receptors and is not essential for the intracellular trafficking and initial clustering of these receptors at the plasma membrane.

The differential role of Gephyrin in glycine and $\mathrm{GABA}_{\mathrm{A}}$ receptor clustering may involve structural variations between the Gephyrin clusters at glycinergic and GABAergic synapses. The expressed Gephyrin variants are highly regulated in a tissue-specific manner by alternative splicing (Kirsch et al., 1993). A detailed mass-spectroscopic analysis of Gephyrin splice variants has revealed that GlyR associated Gephyrin in the spinal cord clearly differs from other neuronal and non-neuronal Gephyrin in its splice cassette usage (Paarman et al., 2006). This finding indicates that Gephyrin splice variants may have distinct tissue-specific roles, which may underlie the differences in $\mathrm{GABA}_{A} \mathrm{R}$ and GlyR clustering. One such mechanism has been postulated, involving a distinct Gephyrin splice 
variant (containing the splice cassette C5), that is highly expressed in hippocampus but not in spinal cord, and has a higher preference for binding to and clustering $\mathrm{GABA}_{\mathrm{A}}$ Rs, as compared to GlyRs (Meier et al., 2004). Therefore C5 cassette-positive splice isoforms of Gephyrin may display selectivity towards $\mathrm{GABA}_{\mathrm{A}} \mathrm{Rs}$, whereas GlyRs are clustered specifically by $\mathrm{C} 5$ cassette-negative Gephyrin variants. Thus, alternative splicing of Gephyrin, as well as other, yet unexplored mechanisms may underlie the differences in Gephyrin-mediated receptor clustering at GABAergic and glycinergic synapses.

\subsection{Assembly of the inhibitory postsynapse}

Due to a lack of adequate techniques that would allow for specific preparation of purified fractions of inhibitory synapses, our current knowledge of the constituents of the inhibitory postsynaptic apparatus is rather limited. Therefore, in contrast to excitatory synapses, which have been characterized in greater detail, molecular models describing the assembly of inhibitory synapses are rudimentary. However, efforts to identify molecules that form the inhibitory postsynaptic density have revealed a few molecular constituents that are specifically localized to inhibitory postsynapses (reviewed by Moss \& Smart, 2001).

\subsubsection{The Gephyrin scaffold}

Among the few molecules that are known to localize specifically to inhibitory postsynapses, Gephyrin is the most well-established scaffolding component. Gephyrin is localized to the vast majority of GABAergic and glycinergic synapses and loss of Gephyrin causes severe deficits in clustering of both $\mathrm{GABA}_{\mathrm{A}}$ and glycine receptors in various areas of the CNS (§ 1.3.1 and 1.3.2). Being a common element for both types of inhibitory synapses, and given its indispensability for the proper function of synaptic inhibition throughout the 
CNS, Gephyrin is a major contributor to the mechanisms that underlie the assembly of inhibitory postsynapses.

Gephyrin is ubiquitously expressed in various tissues. Apart from its synaptic role in the CNS, Gephyrin functions as an enzyme responsible for synthesizing molybdenum cofactor (MoCo), which is required for the activity of certain metallo-enzymes. In fact, evolutionarily, Gephyrin is derived from two prokaryotic genes, MogA and MoeA, which encode enzymes involved in the MoCo biosynthetic pathway. These two genes have fused during evolution, giving rise to the eukaryotic Gephyrin gene. Gephyrin has three domains, an Nterminal G-domain encoded by the MogA, a C-terminal E-domain encoded by MoeA and a C-domain, termed the linker region (reviewed in Fritsch, Harvey \& Schwarz, 2008). Gephyrin lacks PDZ domains that are common to excitatory synapse scaffolds, but is capable of forming lattices through multimerization, presumably by G-domain-mediated trimerization and E-domain mediated dimerization. Via these multimerization steps, Gephyrin is assumed to assemble into hexagonal lattices onto which postsynaptic receptors would be anchored (Kneussel \& Betz, 2000; Sola et al., 2004). Consistent with this model, interefering with G-domain mediated trimerization and E-domain dimerization by introducing charged residues at the oligomerization interfaces impairs Gephyrin clustering (Saiyed et al., 2007).

Gephyrin was initially discovered as a constituent of affinity-purified glycine receptor preparations (Betz et al., 1991). Gephyrin has also been suggested to form associations with cytoskeletal elements, particularly with microtubules. When expressed in cell lines, Gephyrin accumulates in large intracytoplasmic aggregates, termed blobs (Kirsch et al., 1995). It was proposed that these aggregates are nucleated around microtubule organizing centers (Maas et al., 2006). It remains to be determined whether Gephyrin can bind to microtubules directly, or whether this interaction is mediated by Dynein light chain, a microtubule motor protein that can bind to Gephyrin. In fact, GlyRs and Gephyrin were cotransported back and forth between the soma and distal 
dendrites along microtubules via kinesin and dynein motors, providing evidence for active intracellular transport of Gephyrin (Fuhrmann et al., 2002; Maas et al., 2006; Maas et al., 2009). Gephyrin has also been proposed to have links to the actin cytoskeleton, potentially by interacting with actin-binding proteins Profilin and VASP (Giesemann et al., 2003).

\subsubsection{Collybistin, an adaptor for the Gephyrin scaffold}

In non-neuronal cells Gephyrin forms large cytoplasmic aggregates, which stands in stark contrast to its cellular distribution in neurons. It has thus been postulated that a neuron-specific factor allows Gephyrin to associate with the postsynaptic membrane. Collybistin, a brain-specific guanine nucleotide exchange factor, was identified as such a neuronal factor that mediates membrane association of Gephyrin. Upon coexpression of Gephyrin with a non-ubiqutiously expressed splice variant of Collybistin in non-neuronal cells, Gephyrin is redistributed to plasma membrane associated aggregates, much smaller in size (diameter of 0.2-0.5 $\mu \mathrm{m}$ ) than intracytoplasmic blobs, termed submembrane microaggregates (Kins et al., 2000). The critical role of Collybistin in the development of synaptic inhibition has been demonstrated in a mouse genetics study where Collybistin knockout mice show deficits in targeting Gephyrin to the membrane in the hippocampus and amygdala, which consequently leads to impairments of $\mathrm{GABA}_{\mathrm{A}}$ receptor clustering, reduced GABAergic transmission, and altered synaptic plasticity (Papadopoulos et al., 2007). Strikingly, loss of Collybistin does not lead to deficits in clustering of Gephyrin in the spinal cord, nor has it any adverse effects on GlyR clustering or on glycinergic transmission, indicating that compensatory mechanisms may be activated to substitute Collybistin at glycinergic synapses. Furthermore, a point mutation in the SH3 domain of Collybistin (G55A) has been identified in a human patient suffering from hyperekplexia and epilepsy, and overexpression of this Collybistin mutant in cultured neurons results in the loss of postsynaptic Gephyrin and $\mathrm{GABA}_{A} \mathrm{R}$ clusters (Harvey et al., 2004). Additionally, using the Cre-loxP strategy to specifically inactivate the Collybistin gene at different 
developmental stages, it was demonstrated that Collybistin is essential for both the initial formation and the maintenance of GABAergic postsynapses in the mouse hippocampus (Papadopoulos et al., 2008).

Collybistin is a homologue of the Dbl protein family of GDP/GTP exchange factors. There are four splice variants of Collybistin identified in mice (Schema 2), all of which contain a dbl-homology $(\mathrm{DH})$ domain followed by a Pleckstrin Homology $(\mathrm{PH})$ domain. These $\mathrm{DH} / \mathrm{PH}$ tandem domains are commonly found in all known Dbl-family GEFs. DH domains mediate the GDP/GTP exchange on Rho-family GTPases, and the human orthologue of Collybistin, hPEM-2, has been identified as a GEF specific for Cdc42 (Reid et al., 1999). Additionally, the DH domain has been reported to interact with the E-domain of Gephyrin (Grosskreutz et al., 2001). The PH domain of Dbl-family proteins has been implicated in localizing to specific cellular compartments by binding to membrane phosphoinositides (Hyvönen et al., 1995). The PH domain of Collybistin was shown to be critical for the translocation of Gephyrin to the plasma membrane in nonneuronal cells and to synaptic sites in cultured neurons (Harvey et al., 2004). Furthermore, the PH domain of Collybistin has been shown to interact with phosphatidylinositol-3-phosphate (PI3P) (Kalscheuer et al., 2009).

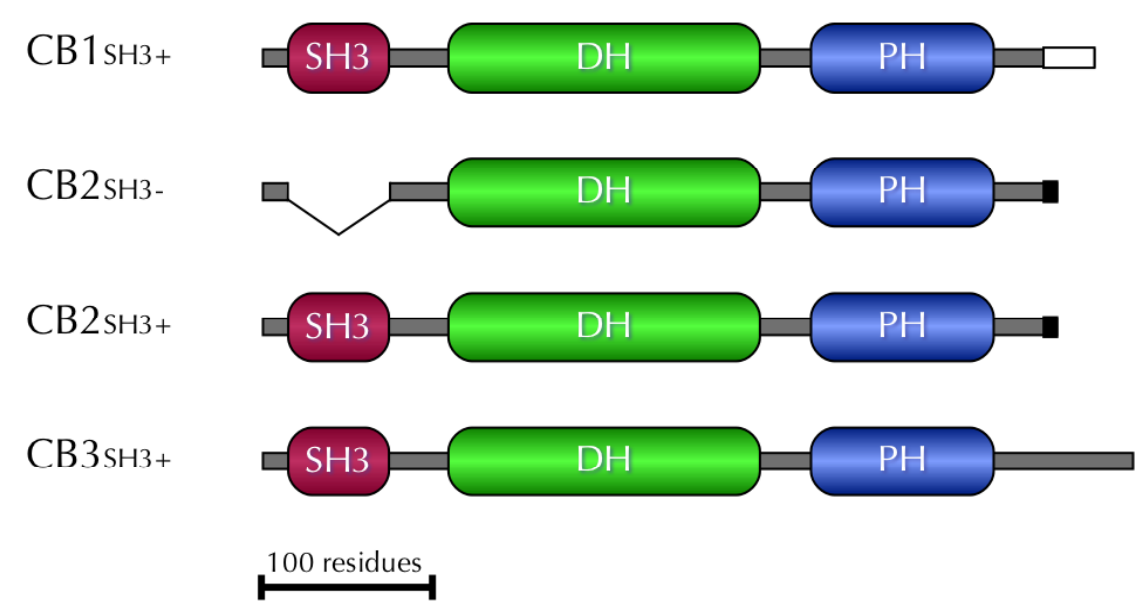

Schema 2: The domain structure and the splice variants of Collybistin: The image depicts the four known splice variants of Collybistin in rodents as identified by Kins et al., (2000) and Harvey et al., (2004). Note that Collybistin CB1-2-3 differ in their C-terminal region, while $\mathrm{CB} 2$ is further divided into two separate splice variants based on the $\mathrm{SH} 3$ domain usage (e.g., $\mathrm{CB} 2_{\mathrm{SH} 3+}$ and $\mathrm{CB} 2_{\mathrm{SH}_{3}-}$. 
In rodents, Collybistin splice variants are divided into 3 main categories based on their C-termini (Harvey et al., 2004; Schema 2). CB1 contains a C-terminal segment that is predicted to form a coiled coil structure, which may be involved in protein-protein interactions (Kins et al., 2000). Another variation exists in the $\mathrm{N}$-terminal region of Collybistin. CB2 exists in two alternatively spliced variants, one containing $\left(\mathrm{CB} 2_{\mathrm{SH}_{3}+}\right)$ and one lacking an N-terminal $\mathrm{SH} 3$ domain $\left(\mathrm{CB}_{\mathrm{SH}_{3}-}\right)$. CB1 and CB3 contain the SH3 domain constitutively (Schema 2).

\subsubsection{NL2, a neuronal factor that activates Collybistin}

Among the four Neuroligin paralogues expressed in rodents, NL2 appears to be the only paralogue that is specifically and constitutively localized to inhibitory synapses (Varoqueaux et al., 2004). In addition, upon clustering by Neurexins, NL2 is the only Neuroligin paralogue to induce GABAergic postsynaptic differentiation (Graf et al., 2004). Corroborating data from in vitro studies, specific deletion of NL2 is detrimental to inhibitory synaptic transmission and caused deficits in the postsynaptic targeting of Gephyrin and $\mathrm{GABA}_{\mathrm{A}}$ receptors at perisomatic synapses in the hippocampus (Chubykin et al., 2007; Hoon et al., 2009; Poulopoulos et al., 2009; Jedlicka et al., 2011). NL2 was shown to associate with Gephyrin in vivo in crosslinked brain preparations (Poulopoulos et al., 2009). This interaction was further validated in fibroblasts and in yeast, where NL2 and Gephyrin were expressed exogeneously, and by GST pulldowns, where soluble forms of cytoplasmic NL2 form complexes with recombinant Gephyrin. These data indicate that NL2 binds to Gephyrin directly and independently of other synaptic proteins. NL2 was shown to interact with the E-domain of Gephyrin. On the NL2 side, a 15-residue stretch, which is highly conserved in all Neuroligins, is necessary to mediate binding to Gephyrin. A tyrosine residue (Y770) within the Gephyrin binding motif appears to be essential for the interaction (Poulopoulos et al., 2009). In addition, NL2 cytoplasmic domain was shown to have signaling properties that lead to the activation of Collybistin by an as yet unknown mechanism (Poulopoulos et al., 2009). 
It was clearly demonstrated that the $\mathrm{SH} 3$ domain acts as an inhibitor of the membrane targeting function of Collybistin. In heterelogous cells, epitope tagged $\mathrm{CB}_{\mathrm{SH}_{3}-}$ has the capacity to redistribute Gephyrin to the submembrane microaggregates, while none of the $\mathrm{SH} 3$ domain containing variants of Collybistin alone $\left(\mathrm{CB}_{\mathrm{SH}_{3+},} \mathrm{CB} 2_{\mathrm{SH}_{3+},} \mathrm{CB}_{\mathrm{SH}_{3+}}\right)$ are able to mediate membrane clustering of Gephyrin (Kins et al., 2000; Harvey et al.; 2004). Since CB2 $2_{\mathrm{SH} 3+}$ and $\mathrm{CB}_{\mathrm{SH}_{3}+}$ are the two splice variants of Collybistin that are dominantly expressed in vivo and due to the fact that in transfected cortical neurons both the SH3-containing and SH3-lacking splice variants of Collybistin can redistribute Gephyrin into synaptic clusters (Harvey et al., 2004), it was proposed that a neuron-specific factor must activate Collybistin by relieving SH3-mediated inhibition. NL2 was suggested to function as such. This Neuroligin paralogue directly interacts with Gephyrin in vivo and it also has the capacity to activate $\mathrm{CB} 2_{\mathrm{SH} 3+}$ and induce submembrane redistribution of the Gephyrin/Collybistin complex, while NL1 and NL3 lack this functional activity (Poulopoulos et al., 2009).

Based on these findings, Poulopoulos et al. (2009) proposed a model for the assembly of inhibitory postsynapses (Schema 3). This model proposes that NL2, as a central organizer of inhibitory postsynapses, accumulates across GABAergic or glycinergic presynaptic terminals to establish nucleation sites that demarcate the deployment of the Gephyrin scaffold at the plasma membrane. Via an as of yet unknown mechanism, NL2 is able to alleviate the SH3 domainmediated inhibition of Collybistin and this allows for membrane tethering of the Gephyrin-Collybistin complex. According to this model, by defining the sites where Gephyrin molecules are clustered, NL2 ensures that inhibitory neurotransmitter receptors are locally accumulated in areas precisely apposed to glycine and GABA release terminals to maintain efficient synaptic transmission. 


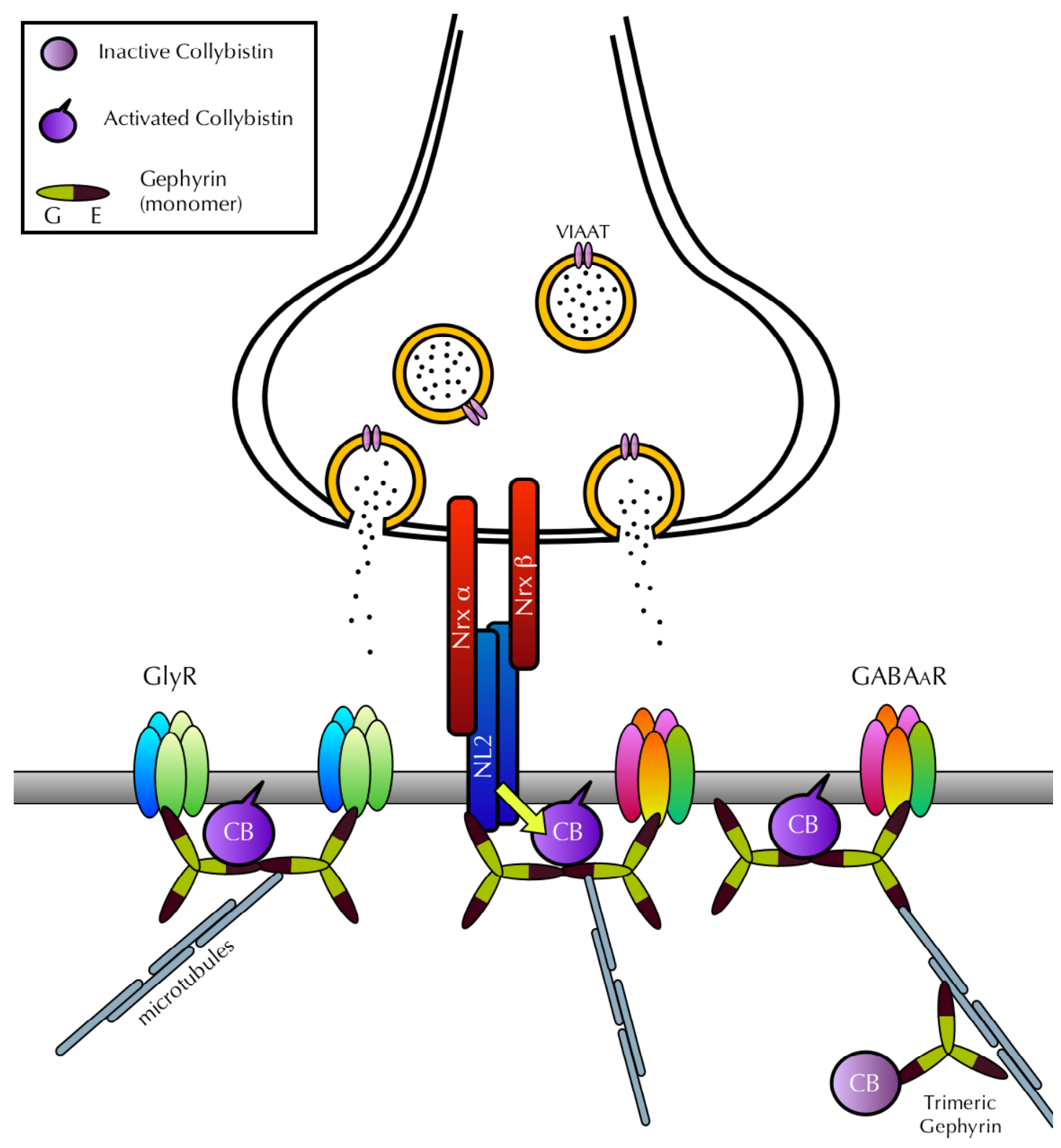

Schema 3: Current assembly model of inhibitory postsynapses: Neuroligin 2 (NL2) dimers are clustered on the surface of the postsynaptic neuron by in trans interaction with $\alpha$ - and $\beta$ neurexins on contacting GABAergic or glycinergic axon terminals. Cytoplasmic GephyrinCollybistin complexes are transiently recruited to plasma membrane sites of Neuroligin 2 clusters via an interaction of the NL2 cytoplasmic domain and the Gephyrin E-domain. Collybistin (in complex with Gephyrin) is activated (yellow arrow) at these nucleation sites by NL2 and the Gephyrin-Collybistin complex is tethered to the plasma membrane via activated Collybistin. Further Gephyrin-Collybistin complexes are recruited to the plasma membrane by NL2, leading to establishment of a membrane tethered postsynaptic Gephyrin scaffold, which in turn clusters GlyRs and $\mathrm{GABA}_{\mathrm{A}} \mathrm{Rs}$ in precise apposition to presynaptic inhibitory transmitter release terminals. 


\subsubsection{The putative roles of NL3 and NL4 at inhibitory synapses}

All Neuroligin paralogues harbor a highly-conserved, 5 residue consensus sequence that functions as a PDZ domain-binding (PDZ-BD) motif (Irie et al., 1997). PDZ domains are typically found in proteins that are responsible for the assembly of cellular junctions and they are often found among multi-protein signaling complexes at excitatory synapses. Through the PDZ-BD motif, NLs are capable of binding to numerous scaffolding molecules, including PDZ domaincontaining proteins, such as PSD-95 (Irie et al., 1997; Bolliger et al., 2001), SSCAM (Hirao et al., 1998) and SHANK (Meyer et al., 2004). Therefore, NLs appear to have a shared feature of associating with the scaffolding molecules of excitatory synapses.

In contrast to excitatory synapses, the protein network at inhibitory postsynapses is not prominently based on PDZ domain-mediated interactions. In fact, another conserved motif was identified in the ICDs of NLs, which mediates interaction with Gephyrin (Poulopoulos et al., 2009). The dual presence of PDZ-binding and Gephyrin-binding motifs indicates that all NLs have a core feature of binding to both excitatory and inhibitory synapse-specific scaffolding molecules. Thereby, in principle, all NLs have the ability to link presynaptic terminals to the postsynaptic scaffolds of both excitatory and inhibitory synapses.

Since all NLs contain a well-conserved Gephyrin-binding motif at their intracytoplasmic domains, it is reasonable to assume that NL1, 3 and 4 may also associate with Gephyrin in vivo. In fact, NL3 and NL4 have been shown to be localized to inhibitory synapses in certain regions and layers of the CNS, while NL1 has been reported to be associated preferentially with excitatory synapses $(\S 1.2 .1)$.

NL3 cannot activate Collybistin or promote synaptic clustering of the Gephyrin scaffold, unlike NL2 (Poulopoulos et al., 2009). Although a detailed analysis of 
NL3 distribution in the CNS is still lacking, the currently available data indicate that NL3 may be associated with both excitatory and inhibitory postsynaptic scaffolds in a tissue specific manner (Budreck and Scheiffele, 2007). It thus remains to be determined whether the distribution of NL3 to excitatory versus inhibitory synapses is a tightly regulated process. In fact, one such mechanism has been proposed by Poulopoulos (2008), hypothesizing that differential phosphorylation of the cytoplasmic tails of Neuroligins may regulate their affinities for Gephyrin. It remains to be tested whether such a mechanism exists in vivo, which would determine specific association of NLs with excitatory versus inhibitory synapses.

Unlike NL1, 2 and 3, NL4 has not been assessed for its potential role in the activation of Collybistin and its capacity to induce postsynaptic targeting of Gephyrin clusters. Since NL4 has recently been shown to be targeted to Gephyrin-positive glycinergic postsynapses in various brain regions (Hoon et al., 2011), it would be interesting to test whether NL4 shares the ability of NL2 in organizing inhibitory postsynapse assembly.

\subsection{Aim of the study}

The previously proposed model for the assembly of inhibitory synapses (Poulopoulos et al., 2009) provided important information regarding the role of NL2 during the differentiation of inhibitory synapses. As a direct interaction partner of Gephyrin and a specific activator of Collybistin, NL2 orchestrates the assembly of the inhibitory postsynaptic apparatus. However, the precise molecular details of this process are unknown. The present study aims to identify the molecular mechanisms underlying the $\mathrm{SH} 3$ domain-mediated inhibition and NL2-induced activation of Collybistin. This information is essential for a coherent molecular model of the assembly of inhibitory synapses, which would eventually cover the gap in our understanding of the development of synaptic inhibition in the mammalian CNS. 


\section{MATERIALS \& METHODS}

\subsection{DNA Constructs}

\subsubsection{Yeast-two-hybrid plasmids}

Yeast-two-hybrid bait constructs of NL1, NL2, NL3 and mouse NL4 intracytoplasmic domains (ICDs) subcloned into the yeast expression vector pLexN were provided by Guido Meyer (Göttingen, Germany). The mutants of NL2 (P764A, P768A and P798stop) were generated and provided by Alexandros Poulopoulos (Göttingen, Germany). Prey constructs of rat $\mathrm{CB}_{\mathrm{SH}_{3+1}}$ $\mathrm{CB} 2 \mathrm{SH} 3+_{3} \mathrm{G} 62 \mathrm{~A}, \mathrm{CB} 2_{\mathrm{SH}_{3},} \mathrm{SH} 3(\mathrm{CB}), \mathrm{SH} 3(\mathrm{CB}) \mathrm{G} 62 \mathrm{~A}$ subcloned into the yeast expression vector pACT2 were provided by Robert Harvey (London, UK). Prey constructs of full-length Gephyrin and S-SCAM subcloned into yeast expression vector pVP16-3 were retrieved from the embryonic day 18 rat brain cDNA library created by Masaya Okamoto (Dallas, USA) (Betz et al., 1997).

\subsubsection{Expression plasmids}

Mammalian expression constructs of HA-NL2 in pcDNA3 (Invitrogen) and HANL3 in pCMV (Clontech Laboratories Inc.) were provided by Stephane Jamain (Göttingen, Germany). The point mutants of HA-NL3 (S799A and S799D) were generated and provided by Alexandros Poulopoulos (Göttingen, Germany). HA$\mathrm{NL} 2_{\mathrm{ECD}}-\mathrm{NL} 1_{\mathrm{ICD}}$ and $\mathrm{HA}-\mathrm{NL} 2_{\mathrm{ECD}}-\mathrm{NL} 4_{\mathrm{ICD}}$ constructs were created from rat and mouse NLs by standard PCR amplification of mutated fragments and subcloning into the pcDNA3 HA-NL2 construct. They include the extracellular domain of rNL2 (residues 1-670), and the transmembrane and intracellular domains of rNL1 (residues 690-843) and mNL4 (residues 754-945), respectively. Above membrane protein constructs are tagged just downstream of the signal peptide sequence, yielding $\mathrm{N}$-terminal epitope tags that are exposed on the cell surface. 
A GFP-Gephyrin expression construct was provided by Theofilos Papadopoulos and Heinrich Betz (Frankfurt, Germany). The myc-CB2 $2_{\mathrm{SH} 3+}$ myc-CB2 $2_{\mathrm{SH}_{3}} \mathrm{G} 62 \mathrm{~A}$, myc-CB2 ${ }_{\mathrm{SH} 3-}$ in pRK5 vector expression constructs were provided by Robert Harvey (London, UK). The HA-Asef expression construct in pcDNA3.1 was provided by Tetsu Akiyama (Tokyo, Japan).

The C-terminal human Immunoglobilin1 heavy chain $(\mathrm{Fc})$ fusion-protein construct of the NL2 ICD in the mammalian expression vector pCMVIG9 (Ichtchenko et al., 1995) was provided by Alexandros Poulopoulos (Göttingen, Germany). The Fc-fusion constructs of NL1, NL3 and NL4 ICDs were generated by standard PCR amplification, using the rat NL1 and NL3, and the mouse NL4 cDNAs (all provided by Stephane Jamain, Göttingen, Germany) as template and subcloning into pCMVIG9 vector. The constructs $\mathrm{NL}_{\mathrm{CD}} \mathrm{Fc}, \mathrm{NL} 2_{\mathrm{CD}} \mathrm{Fc}, \mathrm{NL} 3_{\mathrm{CD}} \mathrm{Fc}$ and $\mathrm{NL} 4_{\mathrm{CD}} \mathrm{Fc}$ express cytoplasmic proteins in mammalian cells.

The Glutathione S-transferase (GST) fused bacterial expression construct for Asef, encompassing the $\mathrm{DH}$ and $\mathrm{PH}$ domains, was provided by Reza Ahmadian (Düsseldorf, Germany). N-terminal GST fusion-protein constructs of $\mathrm{CB}_{\mathrm{SH}_{3+}}$ was created by standard PCR sequence fragment amplification, using the myc$\mathrm{CB} 2_{\mathrm{SH}_{3+}}$ expression construct as a template, and subcloning into pGEX-4T-1 bacterial expression vector, yielding GST-CB2 ${ }_{\mathrm{SH}_{3+}}$ construct. The GST$\mathrm{CB} 2_{\mathrm{SH}_{3}+} \Delta \mathrm{SH} 3$ and GST-CB $2_{\mathrm{SH}_{3}+} \Delta \mathrm{PH}$ constructs were generated by deleting the portions from the ORF of $\mathrm{CB}_{\mathrm{SH}_{+}}$between the residues 20-71 and 325-440, respectively, by using site directed mutagenesis methods (see below).

Mutant variants of the above constructs were produced by performing full plasmid amplification with 35-50 bp fully overlapping primers encompassing the site of mutagenesis and encoding the desired mutation(s). PCRs were performed with high fidelity pfu DNA polymerase (Stratagene) and thermocycler protocols with the following parameters: 
Annealing $\mathrm{T}\left({ }^{\circ} \mathrm{C}\right)$ :

Elongation t (sec): Iterations
68

120 per kb of plasmid

15

Sequences of the complete open reading frames (ORFs) of the products were verified by DNA sequencing.

\subsection{Antibodies and Labeling Reagents}

\subsubsection{List of primary antibodies and working dilutions}

The following primary antibodies were used throughout the study. In the following table 'antigen' refers to the protein or peptide epitope that was used to immunize hosts. 'Serum/clone' refers to the serum designation number of polyclonal antibodies or clone designation number of monoclonal antibodies. 'Source' refers to the commercial provider or creator of the antibody. 'Host' refers to the species of animal that the antiserum came from; monoclonal antibodies are designated as such and are derived from mouse. The last two columns of the table refer to the working dilutions used in immunoblotting with enhanced chemiluminescence readout ('ECL blot') or in immunocytochemistry labeling of cultured cells ('ICC').

\begin{tabular}{|l|l|l|l|l|l|}
\hline Antigen & Serum/clone & Source & Host & ECL Blot & ICC \\
\hline C-myc & $9 E 10$ & Sigma & monoclonal & & $1: 1000$ \\
\hline HA & SG77 & Zymed & Rabbit & & $1: 2000$ \\
\hline Gephyrin & 3 B11 & Synaptic Systems & monoclonal & $1: 3500$ & $1: 4000$ \\
\hline Neuroligin 2 & 799 & F.Varoqueaux & Rabbit & $1: 7000$ & \\
\hline Neuroligin 3 & 804 & F.Varoqueaux & Rabbit & $1: 7000$ & \\
\hline Neuroligin 4 & 75 & S.Jamain & Rabbit & $1: 1000$ & \\
\hline GST & GST-2 & Sigma & monoclonal & $1: 2000$ & \\
\hline Collybistin & & Transduction Labs & monoclonal & $1: 250$ & \\
\hline VIAAT & & Synaptic Systems & guinea pig & & $1: 1000$ \\
\hline Human-IgG-Fc & & Pierce & Rabbit & $1: 2000$ & \\
\hline Myc & & Sigma & Rabbit & & $1: 1000$ \\
\hline Phospho-NL & & A. Poulopoulos & Rabbit & $1: 1000$ & \\
\hline
\end{tabular}




\subsubsection{Conjugated antibodies and reagents}

Goat-derived antibodies with species-specific affinities for immunoglobulins conjugated to horseradish peroxidase (Bio-Rad and Jackson Biochemicals) were used for ECL immunoblotting at a dilution of 1:10000. Alexa Fluor 488, 555 and Cy5 conjugated goat secondary antibodies were used for immunocytochemistry at a dilution of 1:1000.

\subsection{Yeast two hybrid assays}

The LexA yeast-two-hybrid system was used for small-scale assays with a selection of bait and prey constructs. Bait constructs are in yeast expression vector pLexN, fusing the DNA-binding region of LexA and a SV40 large Tantigen nuclear localization signal to the inserted sequence. Prey constructs are in yeast expression vector pVP16-3 or pACT3, fusing the VP16 acidic activation or GAL4 activation domains to the inserted sequence, respectively. Interaction of prey and bait fusion constructs will lead to a functional transcription activation complex and to report gene expression under the control of LexA promoter.

Assays were performed using small-scale co-transformation with the indicated bait and prey constructs along with Salmon sperm carrier DNA by a lithium acetate method, as described in Betz et al., (1997), into auxotrophic Saccharomyces cerevisiae strain L40, lacking metabolic genes for trpytophane, leucine and incubated at $30^{\circ} \mathrm{C}$ for 3 days. Prototropic colonies that emerged represented clones transformed for both bait and prey constructs.

Prey-bait interaction leads to the expression of reporter genes under the control of LexA. Interaction was assessed in co-transfected clones by examining $\beta$ galactosidase activity of LacZ reporter gene expression (Vojtek et al., 1993). The 
readout is based on the catabolism of the chromogenic compound X-gal, making the $\beta$-galactosidase assay visible.

\subsection{Cell culture and transfection}

\subsubsection{Cell-line culture and transfection}

The COS7 cell-line, a fibroblast line derived from African green monkey kidney cells, and the HEK 293T cell-line, an epithelial line derived from human embryonic kidney cells transformed with large T-antigen, were used in this study. Both cell lines were maintained in plastic tissue culture dishes with highglucose Dulbecco's Modified Eagle's Medium (DMEM) (Gibco), supplemented with $10 \%$ fetal calf serum (Gibco), in a $37^{\circ} \mathrm{C}$ humid incubator with $5 \%$ ambient $\mathrm{CO}_{2}$. Passaging was performed using standard procedures of trypsin mediated detachment and dissociation of confluent cultures. For immunocytochemistry, COS7 cells were transfected with the FuGENE6 (Roche) reagent by standard lipofection procedures. For production of recombinant Fc fusion proteins of NL1-4 ICDs, HEK293T cells were transfected with linear polyethylenimine (PEI) MW 25,000 (Polysciences, Inc.) (Protocol provided by Anna Suska, Göttingen). For this purpose, $5 \mu \mathrm{g}$ of plasmid DNA were mixed with $22.4 \mu \mathrm{l}$ of $7.5 \mathrm{mM}$ linear PEI in a $100 \mu \mathrm{l}$ of $150 \mathrm{mM} \mathrm{NaCl}$ solution and this PEI-DNA solution was added onto a suspension of $7 \times 10^{6}$ HEK293T cells in $10 \mathrm{ml}$ of DMEM. The cell suspension was then plated on plastic cell culture plates with $10 \mathrm{~cm}$ diameter and the cells were let grow for 2-3 days until 100\% confluency was achieved.

\subsubsection{Primary neuron culture and transfection}

Primary neuron cultures were prepared from hippocampi of neonatal mice. The hippocampi were treated with 10units/ml papain (Worthington) in DMEM (Gibco) containing $0.2 \mathrm{mg} / \mathrm{ml}$ Cystein, $1 \mathrm{mM} \mathrm{CaCl}$ and $0.5 \mathrm{mM}$ EDTA, for 45 minutes at $37^{\circ} \mathrm{C}$, followed by a 15 -minute incubation with the stop solution 
containing $2.5 \mathrm{mg} / \mathrm{ml}$ Albumin, $2.5 \mathrm{mg} / \mathrm{ml}$ Trypsin inhibitor and 10\% FCS (Gibco) in DMEM (Gibco). After triturating mechanically, the cells were plated on an astrocyte layer at a density of 30.000 cells $/ \mathrm{cm}^{2}$. Neurons were cultured in Neurobasal medium (Gibco) supplemented with B27 (Gibco), glutamax (Gibco) and Penicillin/Streptomycin (Roche).

Dissociated hippocampal neurons were transfected by the $\mathrm{Ca}^{2+}$-Phosphate method at day-in-vitro 4 (DIV4) via the CaPhos Kit (Clontech) according to the manufacturer's instructions. In order to achieve sufficient yields for transfection, the DNA-Ca ${ }^{2+}$-Phosphate precipitate was dissolved in $\mathrm{HBSS}$ with $10 \% \mathrm{CO}_{2}$, as suggested by Jiang \& Chen (2006).

\subsection{Recombinant Protein Binding Assays}

\subsubsection{GST-fusion proteins}

To express N-terminal GST fused $\mathrm{CB} 2_{\mathrm{SH}_{3}}$ and its mutants, BL21DE3 strain E.coli were subjected to electroporation mediated plasmid transformation with the respective constructs in pGEX-4T-1. Luria broth cultures of transformants in the logarithmic growth phase were induced for recombinant protein expression with $0.5 \mathrm{mM}$ IPTG for $24 \mathrm{~h}$ at $16^{\circ} \mathrm{C}$. The cultures were subsequently harvested in Phosphate Buffered Saline (PBS) and cell lysates were prepared by a combination of enzyme treatment with Lysozyme and DNase I, and sonication with a Labsonic $U$ Sonifier (Braun). A standart mix of Leupeptin $(1 \mu \mathrm{M})$, Aprotinin $(1 \mu \mathrm{g} / \mathrm{ml})$ and PMSF $(100 \mu \mathrm{M})$ protease inhibitors were present in these and all subsequent cell homogenization or lysis procedures. The lysate supernatant was prepared by centrifugation with Heraeus Varifuge 20RS in Heraeus Sepatech HFA1350 Centrifuge Rotor at 12.500 rpm for 45 minutes.

Lysate supernatants were used as input for standard glutathione-affinity purification. Glutathione coupled to Sepharose beads (Amersham) was 
incubated with the input samples according to manufacturer's protocol. Beads loaded with purified protein were used directly for GST-fusion protein binding assays. As input, transfected HEK293T cells expressing cytoplasmic Fc-fusion proteins of ICDs of NL1-4 and endogenous Gephyrin were homogenized using rotating glass/Teflon homogenizers or a cell cracker (EMBL), with 5-10 $\mu \mathrm{M}$ clearance, in Tris/HCl-based buffers containing $50 \mathrm{mM} \mathrm{NaCl}$ at $\mathrm{pH} 7.4$ without the use of detergent. The cell debris was removed by high-speed centrifugation with a table-top centrifuge. Beads were incubated under varying conditions with the supernatants of the homogenates above in the presence of $1 \%$ TritonX-100. Elution of bound proteins was performed directly in Laemmli sample buffer (LSB). Samples were prepared on blots by standard SDS-PAGE in Tris-Glycine gels and nitrocellulose electroblot methodologies (Laemmlli et al., 1970; Towbin et al., 1979). Blots were examined for the presence of Gephyrin and Fcfusion proteins of NL1-4 using anti-Gephyrin, anti-Human-IgG-FC and anti-NL2 immunoblotting.

\subsection{Protein-Lipid binding assays}

Recombinant GST-CB2 $2_{\mathrm{SH}_{3}+}$ and its mutant variants were purified with batch methods as described in $\S 2.5 .1$. The proteins bound to the beads were eluted in Tris Buffer containing 50mM Tris- $\mathrm{HCl}, \quad 1 \%$ 3-[(3-cholamidopropyl)dimethylammonio]-1-propanesulfonate (CHAPS), $1 \mathrm{mM} \mathrm{DTT,} 150 \mathrm{mM} \mathrm{NaCl}$, $50 \mathrm{mM}$ Reduced Glutathione at $\mathrm{pH}$ 8.0. The proteins were dialyzed against the same buffer without the detergent using the Slide-A-Lyzer dialysis cassettes (Pierce) with $10 \mathrm{kDa}$ cutoff.

PIP-Strips (Echelon) were used to detect phosphoinositide specificity of Collybistin. The membranes were blocked with $1 \%$ milk in PBST $(0.1 \%$ Tween20). $2 \mu \mathrm{g}$ of protein of interest was added in the blocking buffer and gently agitated for $1 \mathrm{~h}$ at room temperature. After washing three times with the blocking buffer, the bound proteins were detected with standard dot-blot techniques 
using an anti-GST antibody (§ 2.2.1). The custom made Phosphatidyl-inositol-3phosphate (PI3P) strips were prepared by spotting $1 \mu \mathrm{I}$ of 250, 125, 50 and $25 \mathrm{mM}$ of PI3P in chloroform onto the strips of Hybond-C-extra membranes (GE Healthcare) and the assay is repeated as described above for PIP-strips. For PI3P/NL2 cospotting assay, strips of Hybond-C-extra membranes were cospotted with $0.5 \mu \mathrm{g}$ of PI3P and varying amounts $(750,375,150,0 \mathrm{ng})$ of recombinant NL2 cytoplasmic domain (NL2cyt) (provided by Bodo Sander and Hermann Schindelin, Würzburg). The bound proteins were detected as described above assay is repeated as described above for PIP-strips and custom-made PI3P strips.

\subsection{Crosslinking and identification of protein complexes}

\subsubsection{Preparation of crosslinked brainstem and spinal cord material}

Crosslinking was performed on post-nuclear homogenates from adult mouse spinal cord and brainstem (mixed) in PBS. Homogenates were incubated on ice for $20 \mathrm{~min}$ with $20 \mu \mathrm{M}$ of the cleavable, homobifunctionalm membrane permeable $12 \AA$ crosslinker Dithiobis[succinimidylpropionate] (DSP) (Pierce). Crosslinker was quenched in Tris $1 \mathrm{M}, \mathrm{pH} 7.5$, before the material was centrifuged at $21000 \mathrm{~g}$ for $15 \mathrm{~min}$. Proteins from pelleted membranes were extracted from pellets with $1 \%$ SDS in TNE buffer (Tris 50mM, NaCl 150mM, EDTA $5 \mathrm{mM}$, Leupeptin $1 \mu \mathrm{M}$, Aprotinin $1 \mu \mathrm{g} / \mathrm{ml}$ and PMSF 100 $\mu \mathrm{M})$.

\subsubsection{Immunoprecipitation of crosslinked complexes}

SDS extracts of crosslinked proteins were diluted with 7 volumes of TNE with $1 \%$ TritonX-100, producing a micell system of $0.875 \%$ TritonX-100 and $0.125 \%$ SDS that does not denature antibodies. Neuroligin-containing crosslinked adducts were immunoprecipitated from mixed spinal cord and brainstem homogenates using paralogue specific antibodies (anti-NL2 or anti-NL4) listed in $\S 2.2 .1$. After overnight incubations at $4^{\circ} \mathrm{C}$ of the extracts with antibodies, $\lg \mathrm{G}$ 
containing complexes were recovered by a $5 \mathrm{~h}$ incubation at $4{ }^{\circ} \mathrm{C}$ with Protein $\mathrm{A}$ coupled to Sepharose beads. Beads were washed 6 times in $0.875 \%$ TritonX$100 / 0.125 \%$ SDS TNE buffer and proteins were eluted in LSB with $7.5 \% \beta$ mercaptoethanol to reduce protein disulfide bonds and cleave the crosslinker. Samples were subjected to standard SDS-PAGE on Tris-glycine gels and immunoblotted on nitrocellulose membranes using antibodies from $\S 2.2 .1$ to detect crosslinked complex components.

\subsection{Immunolabeling of transfected neurons and cell lines}

\subsubsection{Immunolabeling of transfected cell lines}

Transiently transfected COS7 cells were plated directly onto glass coverslips. Cells were fixed $12 \mathrm{~h}$ post transfection in $4 \%$ PFA, $5.5 \%$ sucrose in $100 \mathrm{mM}$ phosphate buffer $\mathrm{pH} 7.4$ (PB) for $10 \mathrm{~min}$ at room temperature. Samples were blocked with $5 \%$ normal goat serum and $0.1 \%$ gelatin in PB and incubated overnight at $4^{\circ} \mathrm{C}$ with anti-HA $(\S 2.2 .1)$ antibodies for surface labeling of transmembrane proteins. After permeabilization with $0.1 \%$ TritonX-100, the samples were further incubated for $3 \mathrm{~h}$ at room temperature with relevant antibodies from $\S 2.2 .1$ to detect intracellular proteins. Secondary antibody staining was performed for $1 \mathrm{~h}$ at room temperature by using anti-isotypic fluorophore (Alexa-555 and Cy5) conjugated antibodies.

Cell line samples were imaged using an inverse Leica DMIRE2 microscope equipped with an HCX PL APO 63x oil-immersion objective lens and connected to a Leica TCS SP2 AOBS confocal laster scanning setup. Intensity correlation analysis was performed on the images using the Image software $(\mathrm{NIH})$. Briefly, a Gaussian blur was applied to the images using a kernel size of 1 pixel. The HA channel was thresholded to omit pixels coming from the extracellular field. Standard Pearson's correlation coefficient was evaluated in the thresholded fields using the Intensity Correlation Analysis plugin for ImageJ 
from Tony Collins (Wright Cell Imaging Facility, Toronto, Canada) and Elise Stanley (C\&MB, TWRI, Toronto, Canada).

\subsubsection{Immunolabeling of transfected neurons}

Hippocampal neurons from Collybistin $\mathrm{KO}$ mice as noted in $\S 2.4 .2$ were plated on continental astrocyte layers on gelatin coated glass coverslips at a density of 30.000 cells $/ \mathrm{cm}^{2}$. Neurons were fixed at DIV12 in 4\% PFA, 5.5\% sucrose in 100mM phosphate buffer $\mathrm{pH} 7.4$ (PB) for $7 \mathrm{~min}$ at room temperature. Samples were blocked with 5\% normal goat serum and $0.1 \%$ gelatin in PB and permeabilized with $0.1 \%$ TritonX-100. Neurons were stained with primary antibodies in 5\% normal goat serum, $0.1 \%$ gelation and $0.1 \%$ TritonX-100 in PB for $3 \mathrm{~h}$ at room temperature, followed by a $1 \mathrm{~h}$ secondary antibody incubation using anti-isotypic fluorophore (Alexa-488, Alexa-555 and Cy5) conjugated antibodies.

\subsection{Statistical analysis}

Statistical analyses were performed using unpaired, two-tailed Student's T-test. Variance is expressed as standard error of the mean. 


\section{RESULTS}

\subsection{Investigation of the roles of NL2 and NL4 at inhibitory synapses}

\subsubsection{NL2 and NL4 specifically interacts with the SH3 domain of Collybistin}

In contrast to NL1 and NL3, NL2 has the capacity to activate the physiologically relevant isoforms of Collybistin that contain the SH3 domain. Therefore a protein-protein interaction assay where the different Neuroligin paralogues are compared with respect to their capacity to bind Collybistin could potentially shed light onto the distinct role of NL2 in the cell-based Collybistin activation assay and potentially at inhibitory synapses. For this purpose, a cross-screen was performed using reporter gene activation as readout of the yeast-two-hybrid $(\mathrm{Y} 2 \mathrm{H})$ system where bait constructs containing full ICDs of rat NL1, NL2 and NL3 were coexpressed with a prey construct containing the full-length rat Collybistin isoform 2 bearing the $\mathrm{SH} 3$ domain $\left(\mathrm{CB}_{\mathrm{SH}_{3}+}\right.$ ) (Figure $1 \mathrm{~A}$ ). LacZ reporter gene activation assays showed that while Gephyrin exhibits promiscuous binding to all three Neuroligin paralogues tested, Collybistin specifically interacts with NL2. This interaction between NL2 and Collybistin, a feature that is not shared by NL1 and NL3, could explain how NL2 can specifically activate Collybistin and regulate targeting of Gephyrin to submembranous sites in COS7 cells, and to inhibitory postsynaptic sites in neurons.

In a parallel $\mathrm{Y} 2 \mathrm{H}$ assay, it was observed that, similar to NL2, the NL4 cytoplasmic tail also interacts with Collybistin (Figure 1B). The colorimetric readout of the $\mathrm{Y} 2 \mathrm{H}$ assay is qualitative and is not informative of the relative affinities of each Neuroligin isoform for Collybistin. Additionally, the minimum 
exposure time required to obtain a positive signal for NL4-Collybistin interaction was beyond the maximum amount of exposure time to avoid bait construct auto-activation for NL1 and NL3. Therefore, it was not possible to compare all four Neuroligin paralogues in a single $\mathrm{Y} 2 \mathrm{H}$ based LacZ reporter gene activation assay to test their interaction with Collybistin. To obtain a quantitative readout, a GST-pulldown assay was performed where a recombinantly expressed GST- tagged full-length Collybistin construct bearing the $\mathrm{SH} 3$ domain was tested against the Fc- tagged cytoplasmic tails of NL1, NL2, NL3 and NL4 expressed in and extracted from HEK293T cells (Figure 1C). In agreement with the $\mathrm{Y} 2 \mathrm{H}$ assay, the GST-pulldowns also indicated that NL2 and NL4, unlike NL1 and NL3, interact with Collybistin.

A

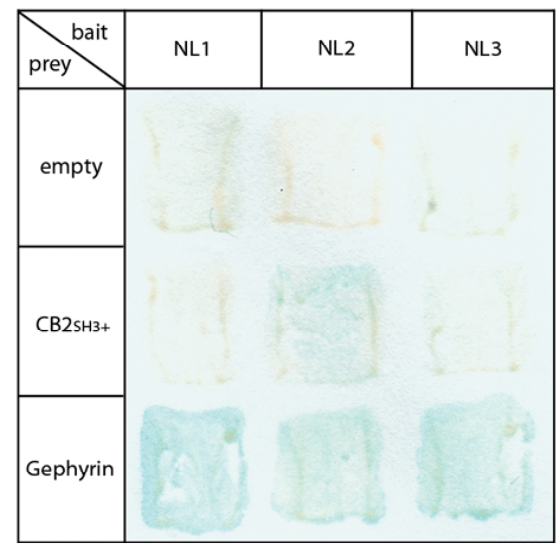

B

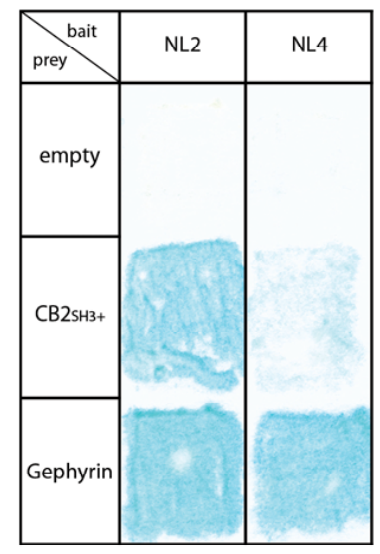

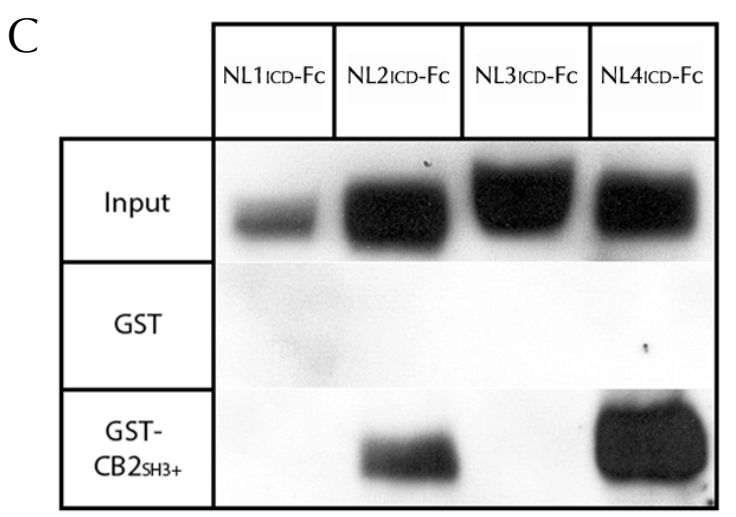

Figure 1: NL2 and NL4 interact with Collybistin: (A) Yeast-two-hybrid (YTH) assays with NL1-3 ICD bait constructs against empty prey vector (top row), $\mathrm{CB}_{\mathrm{SH}_{3+}}$ (middle row) and Gephyrin (bottom row). NL2, but not NL1 or NL3, interacts with Collybistin, while they can all promiscuously interact with Gephyrin. (B) YTH assays with NL2 and NL4 ICD bait constructs against empty prey vector (top row), $\mathrm{CB}_{\mathrm{SH}_{3+}}$ (middle row) and Gephyrin (bottom row). Similar to NL2, NL4 interacts both with Collybistin and Gephyrin. (C) GST-pulldown assays with NL1-4 ICD Fc- fusion proteins produced in HEK293T cells (first lane) against GST alone (middle row) and GST-CB2 ${ }_{\mathrm{SH}_{3+}}$ (bottom row). NL2 and NL4, but not NL1 and NL3, interact with Collybistin. 

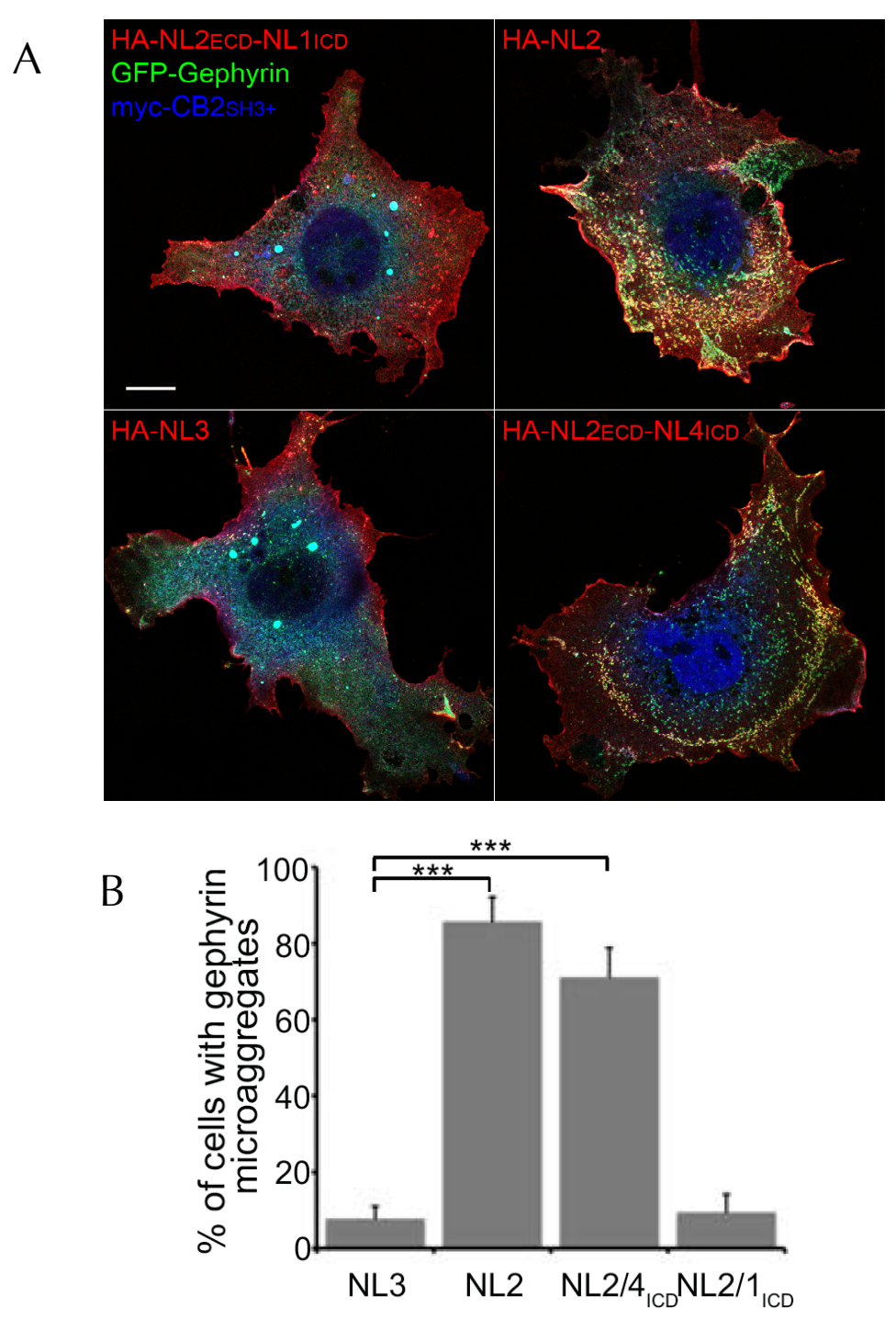

Figure 2: $N L 4_{I C D}$, like $N L 2_{I C D}$ activates Collybistin and induces plasma membrane recruitment of Gephyrin in transfected mammalian cells: (A) COS7 cells were cotransfected with GFP-Gephyrin (green), myc-CB2 $2_{\mathrm{SH} 3+}$ (blue) and a Neuroligin variant (HA-NL2 $2_{\mathrm{ECD}}-\mathrm{NL} 1_{\mathrm{ICD}}$, $\mathrm{HA}-\mathrm{NL} 2, \mathrm{HA}-\mathrm{NL} 3$ or HA-NL2 $2_{\mathrm{ECD}}-\mathrm{NL} 4_{\mathrm{ICD}}$; red). In the presence of $\mathrm{NL} 2_{\mathrm{ECD}}-\mathrm{NL} 1_{\mathrm{ICD}}$ or NL3, GFPGephyrin remains sequestered with myc-CB2 ${ }_{\mathrm{SH}_{3+}}$ in cytoplasmic aggregates. NL2 and $\mathrm{NL} 2 \mathrm{ECD}^{-}$ $\mathrm{NL}_{\text {ICD }}$ activate Collybistin and induce plasma membrane recruitment of Gephyrin in the form of submembrane microaggregates where all three proteins (Collybistin, Gephyrin and NL2 or NL2 ${ }_{E C D}-N L 4_{I C D}$ ) cocluster. Scale bar: $20 \mu \mathrm{m}$. (B) Percentage of COS7 cells triple-transfected with GFP-Gephyrin, myc-CB2 $2_{\mathrm{SH} 3+}$ and a Neuroligin variant $\left(\mathrm{NL} 3, \mathrm{NL} 2, \mathrm{NL} 2_{\mathrm{ECD}}-\mathrm{NL} 4_{\mathrm{ICD}}, \mathrm{NL}_{\mathrm{ECD}^{-}}\right.$ $\left.\mathrm{NL} 1_{1 \mathrm{ICD}}\right)$ where Gephyrin is clustered at submembrane microaggregates: NL3: $7.7 \% \pm 3.4(\mathrm{~N}=3$, $\mathrm{n}=261), \mathrm{NL2}: 85.6 \pm 6.5(\mathrm{~N}=3, \mathrm{n}=285, \mathrm{P}<0.001), \mathrm{NL} 2_{\mathrm{ECD}}-\mathrm{NL} 4_{\mathrm{ICD}}: 71.0 \pm 7.8(\mathrm{~N}=3, \mathrm{n}=276$, $\mathrm{P}<0.001), \mathrm{NL} 2_{\mathrm{ECD}}-\mathrm{NL} 1_{\mathrm{ICD}}: 9.3 \pm 4.8(\mathrm{~N}=3, \mathrm{n}=225)$.

\subsubsection{NL4, like NL2, activates Collybistin and facilitates membrane recruitment of Gephyrin}

Despite the observed similarity of NL2 and NL4 in their capacity to interact with Collybistin, so far it has not been possible to test the capacity of NL4 to activate 
Collybistin due to the lack of a tagged NL4 construct that traffics efficiently to the plasma membrane of heterologous cells. To overcome this problem, chimeric Neuroligin constructs were cloned, where the extracellular domain of NL2 was fused to the transmembrane regions and ICDs of NL1 and NL4. Similar to NL2, coexpression of GFP-Gephyrin and myc-CB2 $2_{\mathrm{SH} 3+}$ along with the HA$\mathrm{NL} 2_{\mathrm{ECD}}-\mathrm{NL} 4_{\mathrm{ICD}}$ chimera also led to the formation of submembrane microaggregates where the three proteins coclustered, indicating that the NL4 cytoplasmic tail shares the ability of the NL2 cytoplasmic tail to bind to and activate Collybistin (Figure 2A). HA-NL3 and HA-NL2 $2_{\mathrm{ECD}}-\mathrm{NL} 1_{\mathrm{ICD}}$ constructs failed to redistribute Gephyrin-Collybistin complexes, which remained in cytoplasmic aggregates, indicating that despite the fact that all Neuroligins bind Gephyrin, the ability to activate Collybistin and stimulate Gephyrin redistribution is restricted to those NLs that can bind to Collybistin.

\subsubsection{The NL2-Collybistin interaction involves the proline rich stretch of NL2 and the SH3 domain of Collybistin}

The proline-rich nature of the ICDs of NL2 and NL4 indicate that binding of NL2 and NL4 to Collybistin might be mediated via a conventional SH3 to PXXP mode of interaction (Lim et al., 1994). In fact, in $\mathrm{Y} 2 \mathrm{H}$ assays it was shown that the $\mathrm{SH} 3$ domain responsible for keeping Collybistin in an inactive state was sufficient to mediate the interaction with NL2 and NL4. On the other hand, the constitutively active Collybistin variant lacking the $\mathrm{SH} 3$ domain $\left(\mathrm{CB} 2_{\mathrm{SH}_{3}-}\right)$ failed to bind to either NL2 or NL4, indicating that the SH3 domain is necessary to mediate binding to both NL2 and NL4 (Figure 3A-B-C). In addition, a G62A substitution in the SH3 domain, corresponding to a G55A substitution in the human orthologue of Collybistin (hPEM-2) associated with severe drug-resistant seizures and hyperekplexia, disrupted NL2 and NL4 binding. It may thus be argued that interaction of the SH3 domain of Collybistin with NL2 or NL4 is involved in relieving the $\mathrm{SH} 3$ domain-mediated inhibition of Collybistin. 


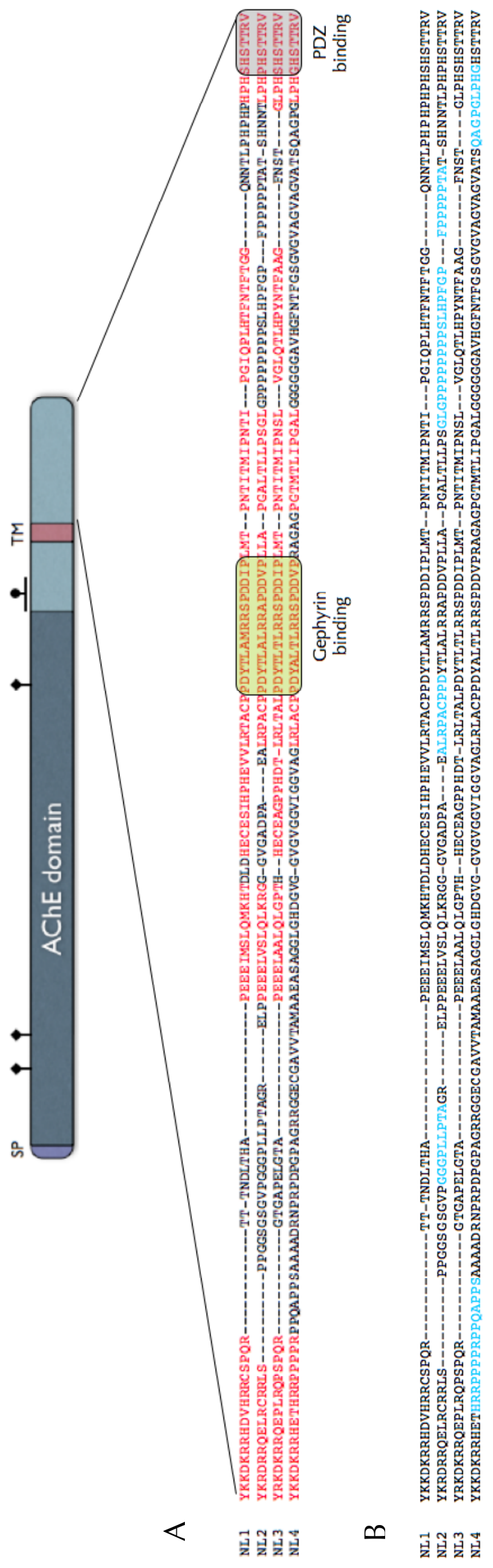

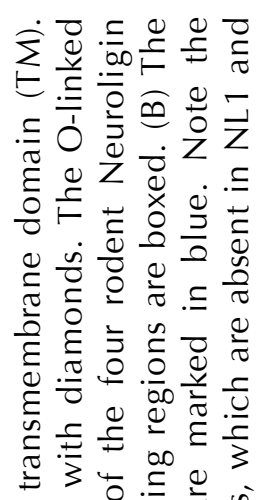

๙

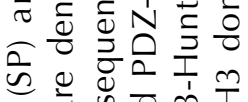

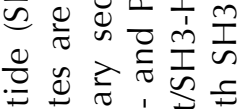

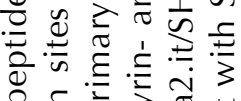

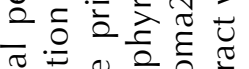

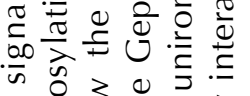

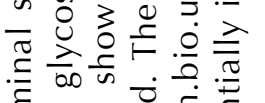
है

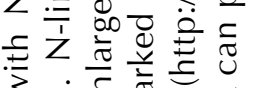

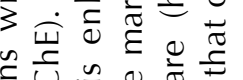

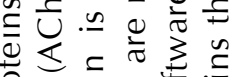
을

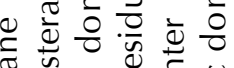

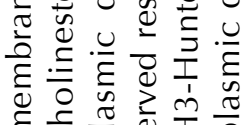

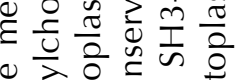

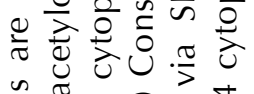
. ơ Z

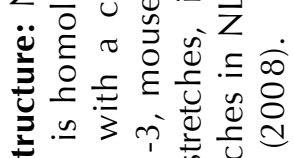

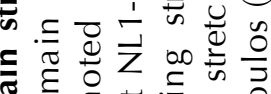
•气

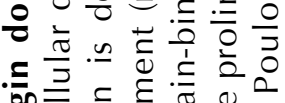

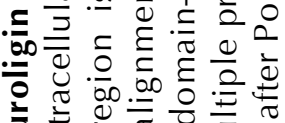

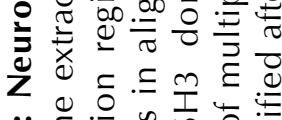

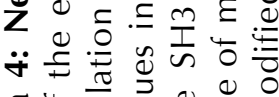
๘ี

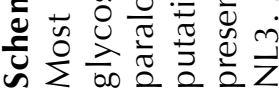


A

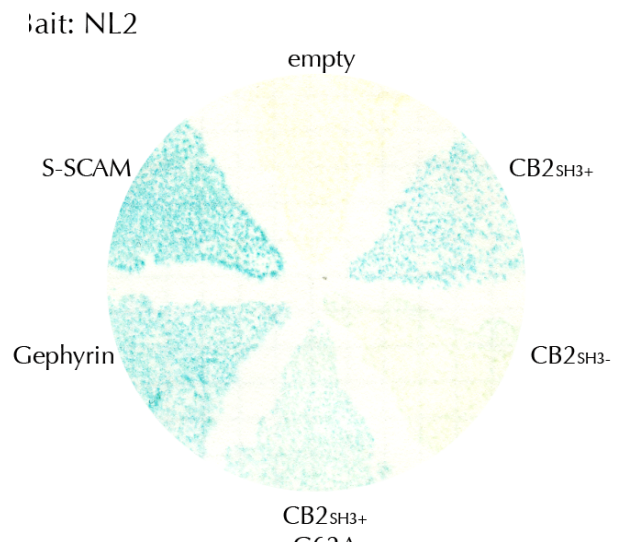

G62A

C

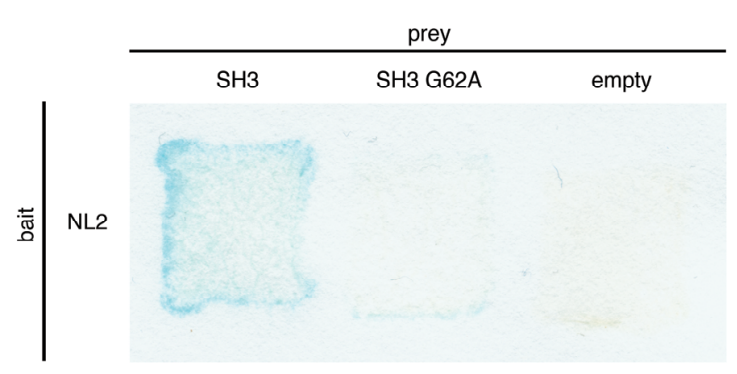

B ait: NL4

empty

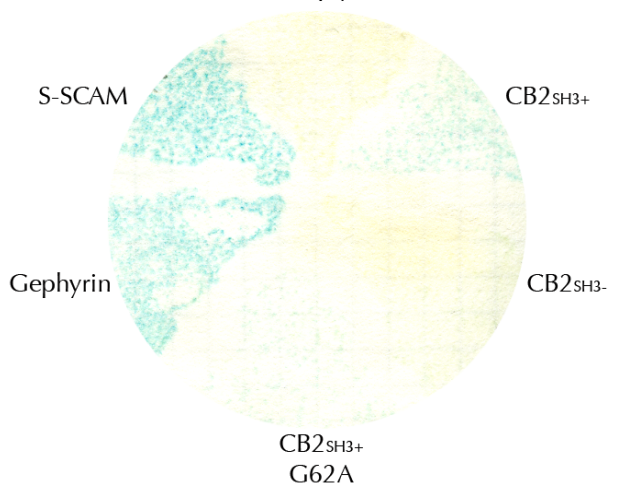

D
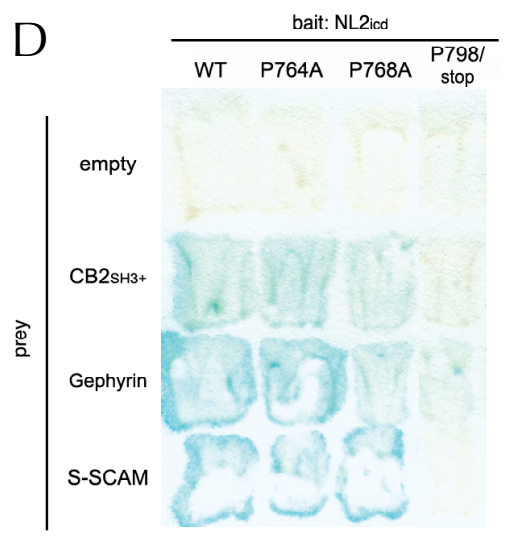

Figure 3: Binding of Collybistin to NL2 and NL4 is mediated by a classical SH3-PXXP mode of interaction: $\mathrm{YTH}$ assays with prey constructs of $\mathrm{CB} 2_{\mathrm{SH}_{3+},} \mathrm{CB}_{\mathrm{SH}_{3-},} \mathrm{CB} 2_{\mathrm{SH}_{3}+} \mathrm{G} 62 \mathrm{~A}$, Gephyrin and S-SCAM against the prey constructs of (A) $\mathrm{NL}_{\mathrm{ICD}}$ and (B) $\mathrm{NL} 4_{\mathrm{ICD}}$. Collybistin $\mathrm{SH} 3$ domain is necessary to mediate binding to NL2 and NL4. The G62A mutation hinders binding if Collybistin to NL2 and NL4. (C) YTH assays with NL2 $2_{\text {ICD }}$ bait construct versus SH3 domain of Collybistin (SH3), SH3/G62A, and empty vector. Collybistin SH3 domain is sufficient to mediate binding to NL2 and G62A mutation hinders this interaction. (D) YTH assays with prey constructs of $\mathrm{CB} 2_{\mathrm{SH}_{3+}}$ Gephyrin and S-SCAM against the bait construct of NL2 $2_{\mathrm{ICD}}$ and its mutants P764A, P768A, P798/stop. Collybistin binding region on NL2 is located downstream from P798.

Multiple sequence alignment of the cytoplasmic tails of the four mouse Neuroligins failed to reveal a proline motif shared specifically by NL2 and NL4 and not NL1 and NL3. However, an SH3 domain interaction site prediction software (SH3-Hunter; http://cbm.bio.uniroma2.it/SH3-Hunter) predicted multiple putative $\mathrm{SH} 3$ interacting sequence stretches within the cytoplasmic tails of both NL2 and NL4, whereas no such putative sites were predicted for NL1 and NL3 (Schema 4). To determine which of the predicted proline motifs is 
responsible for mediating the interaction with the $\mathrm{SH} 3$ domain of Collybistin, three NL2 mutants were tested with $\mathrm{Y} 2 \mathrm{H}$ assays (Figure 3D). The single point mutations P764A and P768A in NL2 did not affect binding to the SH3 domain, while a truncation of the whole C-terminus downstream of P798, did abolish the interaction with Collybistin. This truncation caused the deletion of two long proline stretches (P798-P804 and P813-P817), as well as residues P808 and P811, all of which putatively participate in SH3 domain binding epitopes. The fact that this mutant still binds to Gephyrin indicates that truncation of a large portion of the C-terminal tail of NL2 does not cause expression deficits of this construct. The multiple alignment of Neuroligins downstream of the P798 of NL2 did not reveal the presence of a homologous stretch of proline residues which are conserved in NL4. It is thus very likely that the Collybistin binding region on NL4 is located at another portion of the cytoplasmic tail of this protein.

\subsubsection{Both NL2 and NL4 are capable of establishing high order assemblies with Gephyrin and Collybistin in vivo}

The interaction of NL2 and Gephyrin has been observed in vivo, examined using a chemical crosslinking approach, where postsynaptic NL2-Gephyrin complexes could be detected in mouse brain homogenates upon SDS extraction and immunoprecipitation of endogenous NL2. The specificity of the immunoprecipitation was documented using the brain homogenates of NL2 KO mice (Poulopoulos et al., 2009). Similar to NL2, NL4 has been detected at inhibitory synapses in various brain regions throughout the central nervous system, associated with Gephyrin and GlyRs (Hoon et al., 2011). The putative association of NL4 with Gephyrin was tested in mixed homogenates of mouse spinal cord and brainstem, where glycinergic transmission is prominent. Gephyrin was coimmunoprecipitated with NL4 from chemically crosslinked extracts of spinal cord and brainstem from WT and NL2 KO, but not from NL4 $\mathrm{KO}$ mice (Figure 4A). Collybistin was also detected in coimmunoprecipitates of 
NL2 (Figure 4B) and NL4 (Figure 4A) from crosslinked extracts of spinal cord and brainstem of WT mice. Collybistin was not coimmunoprecipitated from NL2 KO and NL4 KO mice. Overall, these findings show that both NL2 and NL4 can associate into higher order complexes with inhibitory postsynaptic proteins in vivo. Moreover, the slight increase in the detected amount of Gephyrin and Collybistin in the NL4 - coimmunoprecipitated material from the spinal cord and brainstem of NL2 KO mice compared to WT indicates that NL2 and NL4 may have shared functions in the assembly of inhibitory synapses and may compensate for each other in their respective knockouts.

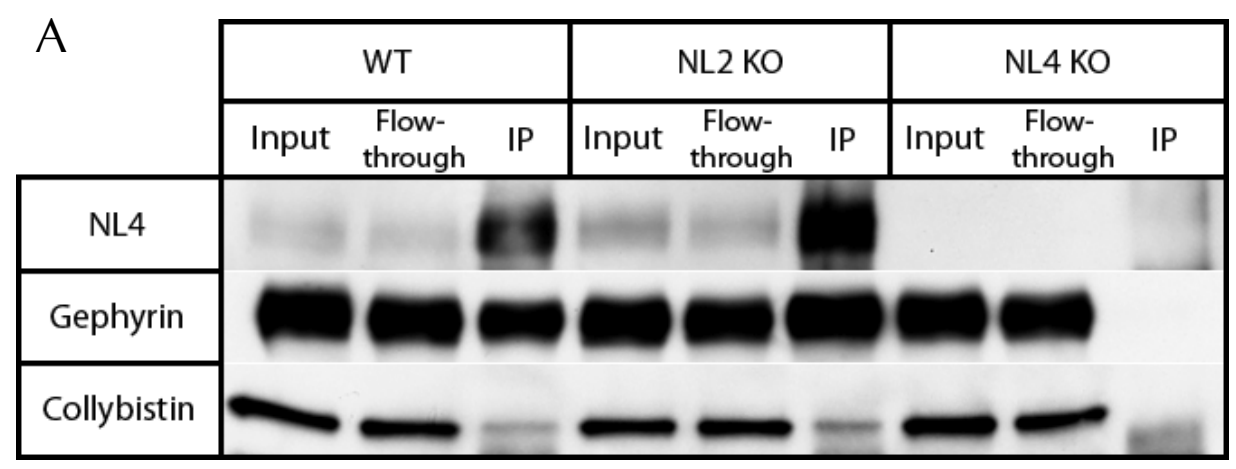

B

\begin{tabular}{|c|c|c|c|c|c|c|}
\hline & \multicolumn{3}{|c|}{ WT } & \multicolumn{3}{|c|}{ NL2 KO } \\
\hline & Input & $\begin{array}{l}\text { Flow- } \\
\text { through }\end{array}$ & IP & Input & $\begin{array}{c}\text { Flow- } \\
\text { through }\end{array}$ & IP \\
\hline NL2 & tha & & & & & \\
\hline Gephyrin & & & & & & \\
\hline Collybistin & $=$ & 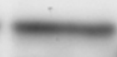 & & 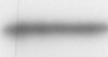 & $=$ & 5 \\
\hline
\end{tabular}

Figure 4: Both NL2 and NL4 assemble into higher order complexes with Gephyrin and Collybistin in vivo: (A) Immunoprecipitation (IP) of endogenous NL4 from crosslinked brainstem (BS) and spinal cord (SC) samples of wild-type (WT), NL2 knockout (NL2 KO) and NL4 knockout (NL4 KO) mice. IP was performed with an NL4-specific antibody. After linker cleavage, Western blot analysis for NL4 (upper row) and Gephyrin (middle row) and Collybistin (lower row) was performed on input extract, flow-through and immunoprecipitated material (IP). Both Gephyrin and Collybistin coimmunoprecipitate with NL4 from the mixed BS and SC homogenates of WT and NL2 KO mice. Note that a nonspecific cross-reactive band of unknown nature that runs below the specific Collybistin band is detected in all immunoprecipitates. (B) IP of endogenous NL2 from crosslinked brainstem and spinal cord samples of WT and NL2 KO mice. IP was performed with an NL2-specific antibody. After linker cleavage, Western blot analysis for NL4 (upper row) and Gephyrin (middle row) and Collybistin (lower row) was performed on input extract, flow-through and immunoprecipitated material (IP). Both Gephyrin and Collybistin coimmunoprecipitate with NL4 from the mixed BS and SC homogenates of WT mice. . Note that a nonspecific cross-reactive band of unknown nature that runs below the specific Collybistin band is detected in all immunoprecipitates. 


\subsubsection{Comparative analysis of $\mathrm{SH} 3$ domains reveals a putative NL2 binding site on Collybistin}

Collybistin has a modular structure comprising $\mathrm{SH} 3, \mathrm{DH}$ and $\mathrm{PH}$ domains, all of which are found in various protein families. The $\mathrm{DH}$ and $\mathrm{PH}$ domains usually exist together as a tandem $\mathrm{DH} / \mathrm{PH}$ module. In the human proteome there is a high number of guanine nucleotide exchange factors that share a similar domain organization with Collybistin, bearing an $\mathrm{SH} 3$ domain adjacent to either the N-terminus ( $\beta$ PIX, $\alpha$ PIX, ASEF, ARHGEF6) or the C-terminus (GEF16, Ephexin, TIM) of the tandem $\mathrm{DH} / \mathrm{PH}$ domains. Additionally, there are other guanine nucleotide exchange factors with more complex domain organizations, which nevertheless carry multiple $\mathrm{SH} 3$ domains located adjacent to the $\mathrm{DH} / \mathrm{PH}$ module (VAV1, VAV2, VAV3, Kalirin, Intersectin1, Intersectin 2) (reviewed in Schiller et al., 2006). Interestingly, characterization of some of these $\mathrm{SH} 3$ domain-containing GEFs, including ASEF and Intersectin, indicated that it is a common feature of the $\mathrm{SH} 3$ domain to confer an inhibitory effect on the enzymatic activity of the DH domain (Zamanian et al., 2003; Mitin et al., 2007; Murayama et al., 2007).

SH3 domains are protein modules that bind to proline rich peptides. The domain is found in a large number of intracellular proteins, many of which are involved in signaling and regulation. The overall structure of the domain is defined by a compact $\beta$ sandwich consisting of five major strands, three of which are connected by variable loops, referred as the RT lop, the N-Src loop and the distal loop; the fourth and fifth strands are separated by a $3_{10}$ helix (Mayer \& Saksela et al., 2005). The Collybistin SH3 domain was aligned with the various $\mathrm{SH} 3$ domains of other signaling and scaffolding proteins (Schema 5) in order to highlight the structurally critical residues in Collybistin that are important for the proper assembly of the $\mathrm{SH} 3$ module such as residues forming the hydrophobic core (labeled in yellow) and residues defining the core ligandbinding site (labelled in green). As seen in the alignment, most of the 
hydrophobic core and ligand binding residues are well conserved in the $\mathrm{SH} 3$ domain of Collybistin. Based on the sequence alignment, the important structural elements, such as the N-Src and RT-loops as well as the hydrophobic core and the core ligand binding site residues on the available NMR structure of the SH3 domain of Collybistin (PDB Code: 1YSQ) were marked (Schema 2A).
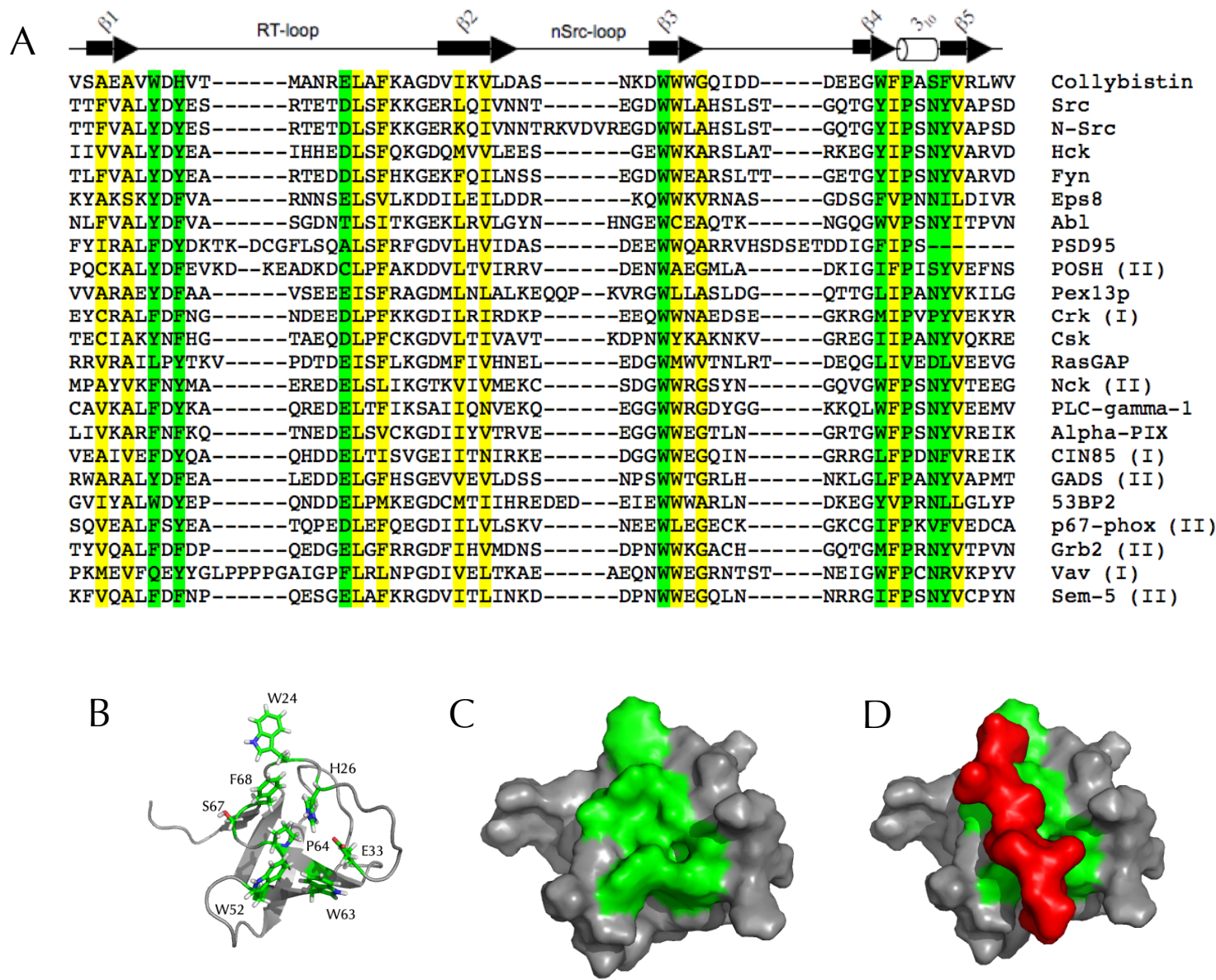

Schema 5: Structure of the SH3 domain: (A) Sequences of $\mathrm{SH} 3$ domains from various different proteins are aligned. At top, positions of $\beta$ strands and the $3_{10}$ helix are indicated, along with the positions of the RT, N-Src, and distal loops. Yellow shading indicates hydrophobic core residues, and green indicates conserved residues defining the core ligand binding site. The alignment is adapted from and modified after Mayer \& Saksela et al., 2005. (B) Ribbon diagram of N-terminal SH3 domain of Collybistin (PDB code: 1YSQ). Sidechains of the core ligand binding-site residues shaded green in (A) are depicted. (C) Surface representation of Collybistin $\mathrm{SH} 3$ in the same orientation as in (B). Core peptide binding site residues are shaded in green. (D) The proline-rich peptide APTMPPPLPP of 3BP-1 (from the structure of Abl tyrosine kinase $\mathrm{SH} 3$ domain in complex with $3 \mathrm{BP}-1$; PDB code: $1 \mathrm{ABO}$ ) was superpositioned onto Collybistin SH3 domain. The structural representations were prepared in MacPyMOL software. 
The proline rich peptides to which the $\mathrm{SH} 3$ domains bind can be divided into two standard classes based on their patterns, class I ([RKHFWY]xxPxxP) or class II (PxxPx[RKH]) (Mayer \& Saksela et al., 2005). Neither NL2 nor NL4 contain patterns that precisely match one of these two classes, but they do contain several PXXP motifs, which correspond to the minimal consensus sequence of a $\mathrm{SH} 3$ proline-rich ligand. In order to test how the NL2 and NL4 cytoplasmic tails may interact with the $\mathrm{SH} 3$ domain of Collybistin, a model $\mathrm{SH} 3$ ligand structure (the proline-rich peptide APTMPPPLPP of 3BP-1 in complex with the Abl tyrosine kinase SH3 domain with 3BP-1 synthetic peptide; PDB code: 1ABO) was used as a structural scaffold onto which the Collybistin SH3 domain was superpositioned (Musacchio et al., 1994; Schema 2B). This structural alignment of two highly similar SH3 domains (58\% homologous and $27 \%$ identical) allowed for the identification of residues in the Collybistin $\mathrm{SH} 3$ domain that are potentially essential for interacting with the proline-rich tails of NL2 and NL4. This analysis pointed to hydrophobic residues W24, W52, W63 and F68 of Collybistin (Schema 7).

\subsubsection{The $\mathrm{SH} 3$ domain of Collybistin may function as an autoinhibitory module}

The crystal structures of homologous guanine nucleotide exchange factors, such as Asef (PDB code 2PZI and 2DX1) and Intersectin-1 (PDB code 3JV3), revealed that in these molecules the $\mathrm{SH} 3$ domain is able to form an extensive interface with the catalytic DH and PH domains to obstruct binding to small GTPases, particularly to Cdc42, thus reducing the GEF activity of both proteins (Mitin et al., 2007; Ahmad et al., 2010). Based on the high similarity of the primary sequences of Asef and Collybistin, it is plausible that the $\mathrm{SH} 3$ domain of Collybistin may also form a similar extensive interface with its $\mathrm{DH} / \mathrm{PH}$ domains. It is well documented that the $\mathrm{SH} 3$ domain of Collybistin imposes an inhibitory effect on the synaptic role of Collybistin in targeting Gephyrin to the plasma membrane, while binding of NL2 to the SH3 domain alleviates this inhibitory 
effect (Poulopoulos et al., 2009). However it remains to be examined whether the $\mathrm{SH} 3$ domain mediated inhibition in Collybistin affects the enzymatic GEF activity of the $\mathrm{DH}$ domain like in Asef and Intersectin-1 or whether the $\mathrm{SH} 3$ domain is responsible for blocking the membrane tethering of the $\mathrm{PH}$ domain.
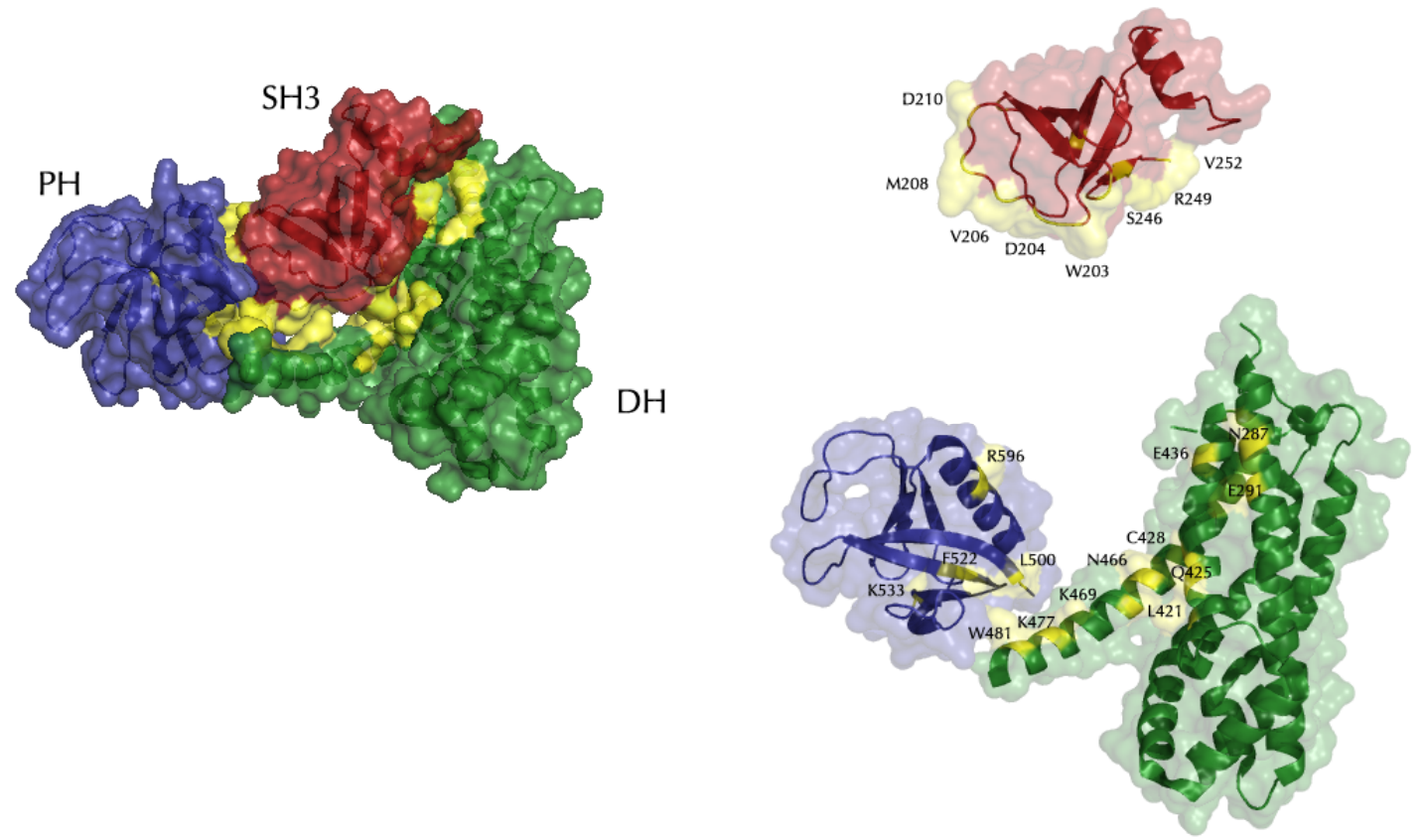

Schema 6: The crystal structure of the autoinhibited Asef: (A) Surface representation of autoinhibited conformation of Asef, based on the available structural information (PDB code: 2PZ1). The residues contributing to the interface between $\mathrm{SH} 3$ (red) and $\mathrm{DH}$ (green) and $\mathrm{PH}$ (blue) domains are depicted in yellow. The structural representations were prepared in MacPyMOL software.

A close look at the crystal structure of Asef (Murayama et al., 2007; Mitin et al.,2007; Schema 6) reveals that the tandem $\mathrm{DH}$ and $\mathrm{PH}$ domains form an arch like structure, which is stabilized by the SH3 domain. W2O3 in the SH3 domain (W24 in Collybistin) is inserted into the hydrophobic pocket formed by L421 (L247 in Collybistin) and V424 (V250 in Collybistin) of the DH domain. M208 (M29 in Collybistin) forms hydrophobic interactions with W481 (W307 in Collybistin) in the DH domain as well as L500 (L326 in Collybistin) and F522 (F348 in Collybistin) in the PH domain, stabilizing the arched conformation of the $\mathrm{DH}$ and $\mathrm{PH}$ domains. On the opposite side of the $\mathrm{SH}$ - $\mathrm{DH}$ domain 
interface, a set of hydrogen bonds forms between the E436 (E262 in Collybistin) and residues R249, V252, N253 (R70, V73, N74 in Collybistin, respectively) of the $\mathrm{SH} 3$ domain. All the other residues forming the interface between the $\mathrm{SH} 3$ and DH/PH domains of Asef are shown in Schema 6.

Based on the available structural information deduced from Collybistin homologues and other $\mathrm{SH} 3$ domain-containing proteins, a mutagenesis screen was designed to elucidate the mechanism of NL2-induced activation of Collybistin. Two different sets of mutations were introduced into the $\mathrm{SH} 3$ and $\mathrm{DH}$ domains of Collybistin. The first set of mutations, including W52A and W63A, was chosen from the pool of residues that are potentially responsible for binding to the proline-rich tails of NL2 (Schema 5). These mutants were generated in order to abolish binding of NL2 to the $\mathrm{SH} 3$ domain and examine whether this interaction is essential for the activation of Collybistin and subsequent membrane targeting of the Gephyrin scaffold. The second set of mutations (including W24A, R70A, E262A) was chosen from the pool of residues responsible for the formation of a putative interface between the $\mathrm{SH} 3$ domain and the $\mathrm{DH} / \mathrm{PH}$ domains, as their analysis may help to explain why the SH3 domain inhibits Collybistin function. Examination of whether Collybistin can be rendered constitutively active by obstructing this putative intramolecular interaction will reveal its potential implication in the inhibiting effect of the $\mathrm{SH} 3$ domain. Towards this end, two mutations in the SH3 domain (W24A, R70A) and one mutation in the $\mathrm{DH}$ domain (E262A) were generated.

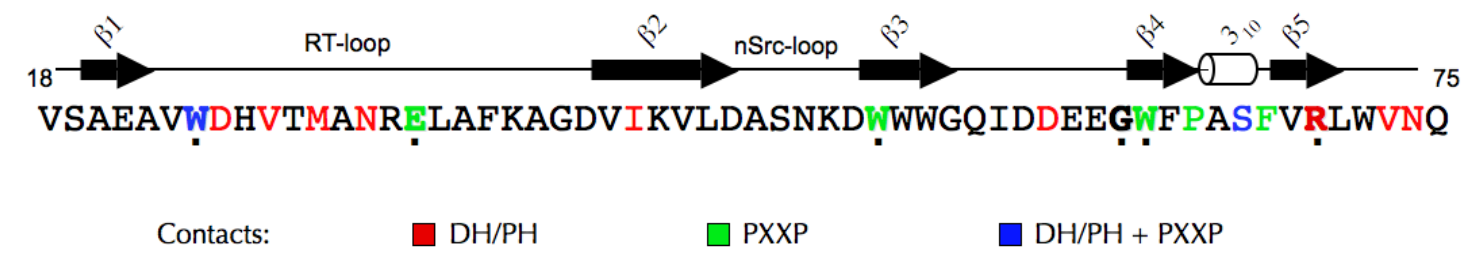

Schema 7: Putative intra- and intermolecular contacts established by Collybistin SH3 domain: Residues of the $\mathrm{SH} 3$ domain of Collybistin that form the putative interface with the $\mathrm{DH} / \mathrm{PH}$ domains are marked in red, while those that are predicted to bind PXXP containing peptides are green. W24 and S67 (blue) are involved in both sets of interactions. The secondary structure of the domain is shown above the sequence. Dots indicate the residues that are substituted by alanines in this study. Modified after Mitin et al., 2007. 
Both pools of mutants were tested using a cell based assay, where GFPGephyrin, HA-NL2 or HA-NL3 and myc-CB2 ${ }_{\mathrm{SH}_{3}+}$ Constructs were co-expressed in COS7 cells and the ability of each Collybistin mutant to target Gephyrin to plasma membrane sites was assessed in the presence of either NL2 or NL3. Since coexpression of HA-NL2 along with GFP-Gephyrin and myc-CB2 $2_{\mathrm{SH} 3+}$ leads to activation of Collybistin and formation of submembrane microaggregates where all three protein components are coclustered (Poulopoulos et al., 2009), it would be interesting to determine whether the Collybistin SH3 domain mutants which are potentially deficient in binding to NL2 (W52A, G62A, W63A) would still show response to the presence of NL2 and be activated. NL3 is another Neuroligin paralogue which was used in this assay. It is deficient in binding to and activating Collybistin, but due to the fact that it has the conserved Gephyrin binding region in its cytoplasmic tail, it can be coclustered with Gephyrin at submembrane microaggregates upon coexpression with $\mathrm{CB} 2_{\mathrm{SH} 3-}$ (Poulopoulos et al., 2009). Therefore, by determining the level of colocalization of plasma membrane targeted Gephyrin and NL3, it would be possible to obtain a readout of intrinsic activity of each Collybistin mutant and observe whether alanine replacement of the residues that are responsible for mediating the putative intramolecular interface between the $\mathrm{SH} 3$ and $\mathrm{DH} / \mathrm{PH}$ domains would lead to auto-activation of Collybistin.

\subsubsection{Interfering with the putative intramolecular interaction renders Collybistin constitutively active}

In the first set of mutagenesis assays, the myc-CB2 ${ }_{\mathrm{SH}_{3+}}$ mutants as well as the non-mutated $\mathrm{CB} 2_{\mathrm{SH}_{3}+}$ and $\mathrm{CB} 2_{\mathrm{SH}_{3}-}$ were coexpressed with GFP-Gephyrin and HA-NL3. As expected, coexpression of myc-CB2 $2_{\mathrm{SH} 3+}$ and GFP-Gephyrin in COS7 cells leads to intracytoplasmic accumulations of Gephyrin, termed blobs (Figure 5, panel 1). Coexpression of NL3 is not sufficient to activate $\mathrm{CB} 2_{\mathrm{SH}_{3}+}$ and rescue Gephyrin from these intracytoplasmic aggregates. However upon coexpression with $\mathrm{CB}_{\mathrm{SH}_{3-1}}$ autonomously membrane targeted Gephyrin- 
A

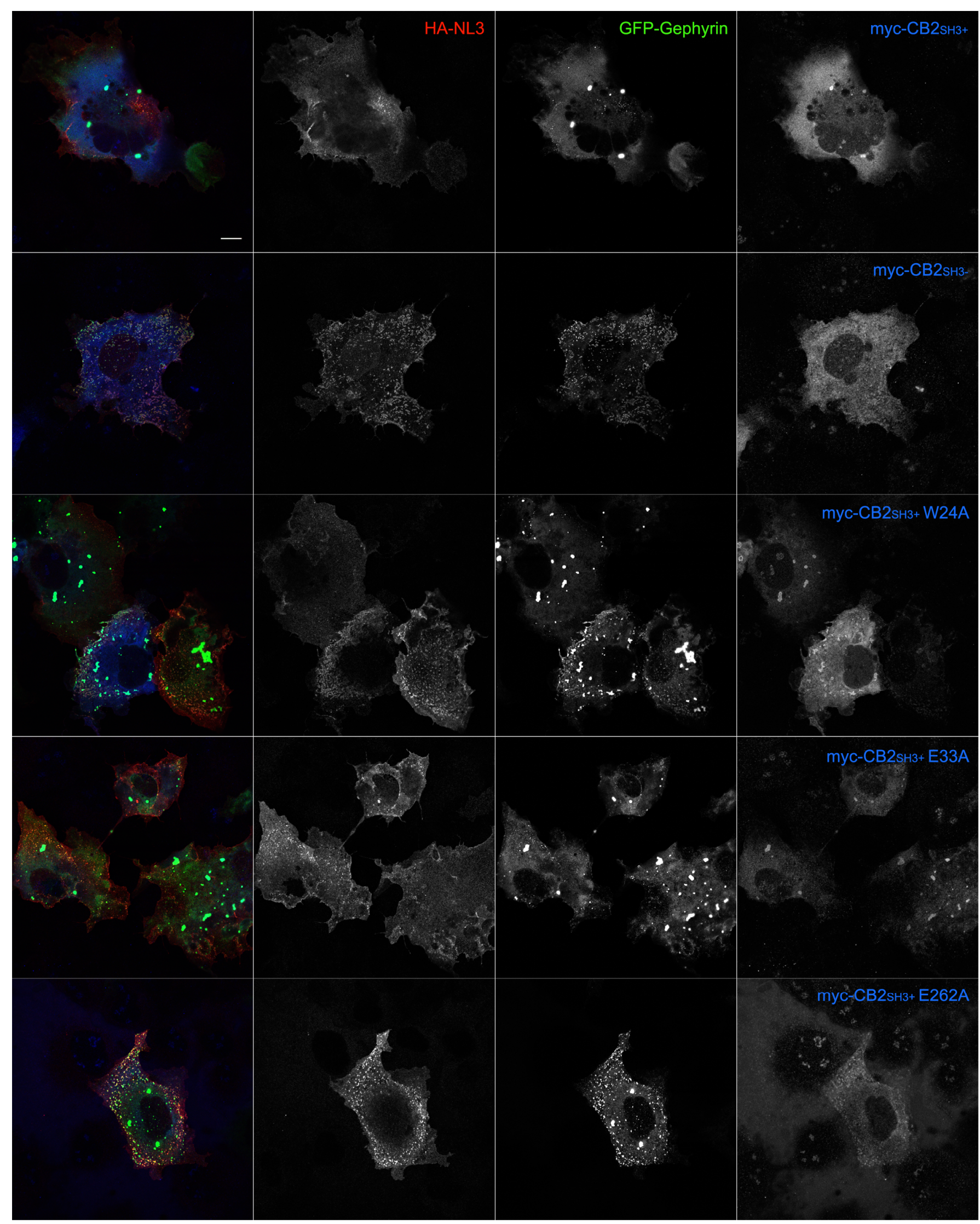




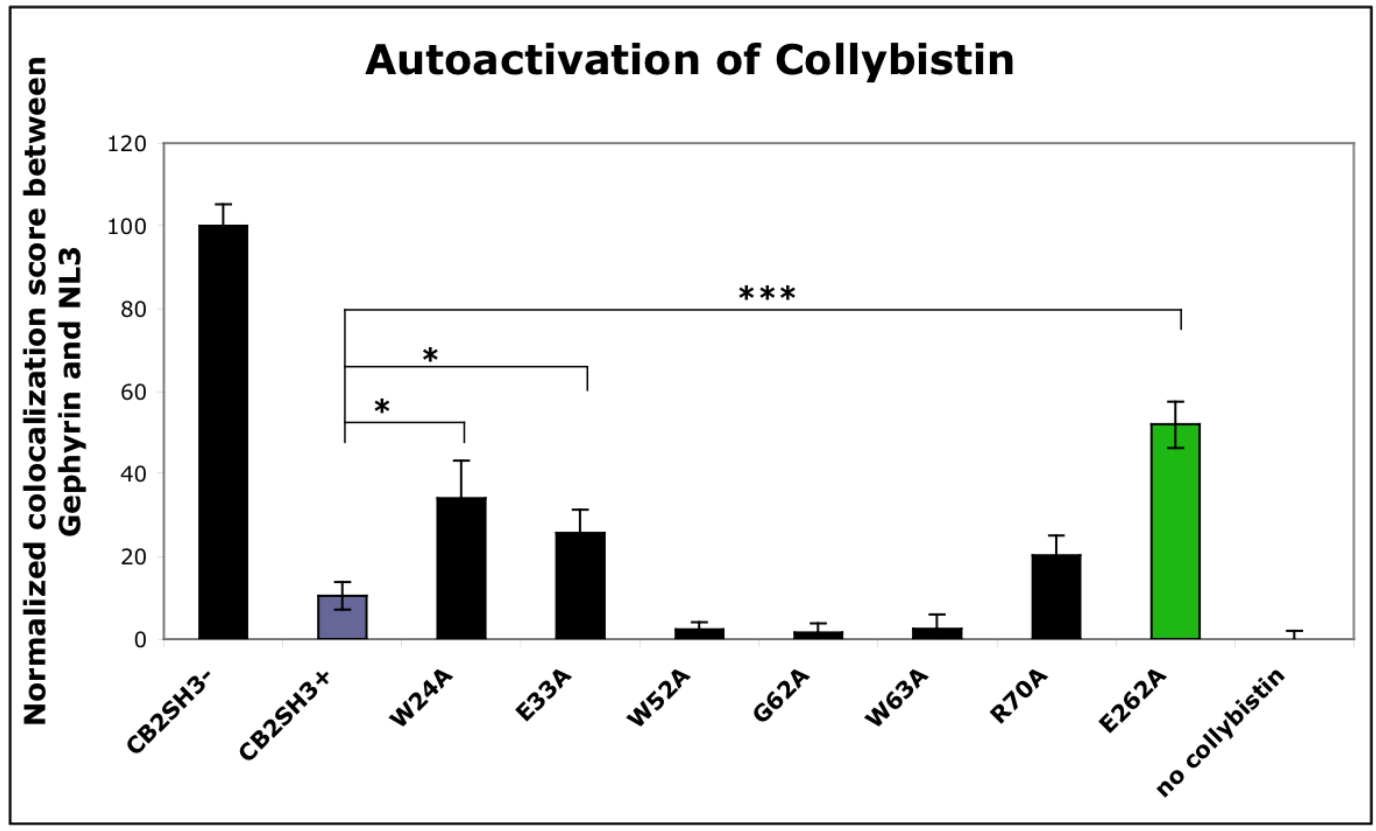

Figure 5: The putative interface formed between $\mathrm{SH} 3$ and $\mathrm{DH} / \mathrm{PH}$ domains is responsible for retaining Collybistin in an autoinhibited conformation: (A) COS7 cells were transfected with HA-NL3 (red), GFP-Gephyrin (Green) and myc-CB2 $2_{\mathrm{SH} 3+}$ its point mutants or myc-CB2 $2_{\mathrm{SH} 3-}$. Among the point mutants tested, myc-CB2 ${ }_{\mathrm{SH}_{3}+} \mathrm{E} 262$ seemed to have the highest autoactivation, and redistributes Gephyrin to submembrane microclusters in the absence of NL2, unlike myc$\mathrm{CB} 2_{\mathrm{SH}_{3+}}$ which stays intracytoplasmically associated with Gephyrin blobs. Scale bar: $20 \mu \mathrm{m}$. (B) The correlation coefficients of GFP- and surface stained HA- signals of GFP-Gephyrin and HANL3, respectively, were measured to quantify the level of autoactivation of each Collybistin mutant. Correlation coefficient values were normalized to the constitutively active Collybistin variant $\left(\mathrm{CB} 2_{\mathrm{SH} 3}\right.$ ) representing $100 \%$ activation, and baselined to samples lacking Collybistin, representing $0 \%$ activation. $\mathrm{CB} 2_{\mathrm{SH} 3+}$ showed significantly reduced activity $(10,56 \% \pm 3.31, \mathrm{~N}=3$, $\mathrm{n}=20)$ compared to mutants $\mathrm{W} 24 \mathrm{~A}(34.10 \% \pm 8.95, \mathrm{~N}=3, \mathrm{n}=15, \mathrm{P}<0.01), \mathrm{E} 33 \mathrm{~A}(25.64 \% \pm 5.60$, $\mathrm{N}=3, \mathrm{n}=15, \mathrm{P}<0.01)$ and $\mathrm{E} 262 \mathrm{~A}(51.90 \% \pm 5.71, \mathrm{~N}=3, \mathrm{n}=15, \mathrm{P}<0.001)$. Conversely, point mutations generated to abolish the interaction between the SH3 domain and NL2 (W52A, G62A, W63A) did not lead to significant changes in the autoactivation of Collybistin.

Collybistin complexes were coclustered with NL3, which contains an extracellular HA- tag that was stained without membrane permeabilization. Interestingly, a group of mutants of Collybistin, namely myc-CB2 ${ }_{\mathrm{SH} 3+} \mathrm{W} 24 \mathrm{~A}$ and E262A, which were predicted to obstruct the putative intramolecular interaction between the $\mathrm{SH} 3$ and $\mathrm{DH} / \mathrm{PH}$ domains, were able to target Gephyrin to the plasma membrane to higher extents as compared to $\mathrm{CB} 2_{\mathrm{SH}_{3}+}$. The correlation coefficients of GFP- and surface stained HA-signals of GFP-Gephyrin and HANL3, respectively, were measured to quantify the level of autoactivation of each Collybistin mutant. Correlation coefficient values were normalized to the 
constitutively active Collybistin variant $\left(\mathrm{CB} 2_{\mathrm{SH}_{3}-}\right)$, representing $100 \%$ activation, and baselined to samples lacking Collybistin, representing $0 \%$ activation. $\mathrm{CB} 2_{\mathrm{SH} 3+}$ showed significantly reduced activity $(10,56 \% \pm 3.31, \mathrm{~N}=3, \mathrm{n}=20)$ as compared to mutants W24A $(34.10 \% \pm 8.95, \mathrm{~N}=3, \mathrm{n}=15, \mathrm{P}<0.01), \mathrm{E} 33 \mathrm{~A}$ $(25.64 \% \pm 5.60, N=3, n=15, P<0.01)$ and E262A $(51.90 \% \pm 5.71, N=3, n=15$, $\mathrm{P}<0.001)$. Conversely, point mutations generated to abolish the interaction between the SH3 domain and NL2 (W52A, G62A, W63A) did not lead to significant changes in the autoactivation of Collybistin. This set of data indicates that a putative intramolecular interaction between the $\mathrm{SH} 3$ and $\mathrm{DH} / \mathrm{PH}$ domains might be instrumental in keeping Collybistin in a closed and inactive conformation.

\subsubsection{NL2-Collybistin interaction is necessary for NL2-mediated Collybistin activation}

In the second set of mutagenesis experiments, the myc-tagged Collybistin mutants were coexpressed with GFP-Gephyrin and HA-NL2 (Figure 6). Similar to what has been observed with NL3, coexpression of HA-NL2, GFP-Gephyrin and myc-CB2 $2_{\mathrm{SH}_{3}-}$ leads to the coclustering of NL2 with the Gephyrin/Collybistin complex at the plasma membrane, due to the association of NL2 with Gephyrin. However, in contrast to NL3, NL2 has the ability to activate $\mathrm{CB} 2_{\mathrm{SH}_{3}+}$ and this activation leads to the membrane recruitment of the Gephyrin/Collybistin complex and coclustering of surface expressed NL2. Interestingly, Collybistin mutants W52A and W63A that were generated to interfere with the interaction between NL2 and the SH3 domain, as well as the G62A mutation, which has already been shown to disrupt NL2 binding to the SH3 domain, were unable to target Gephyrin to the plasma membrane. Similar to the autoactivation assay done with NL3, calculating the relative levels of colocalization between GFPand HA-signals of Gephyrin and NL2, respectively, provided a quantitative assessment of each Collybistin mutant in their response to NL2 activation. Once again, values were normalized to those obtained with the constitutively active 


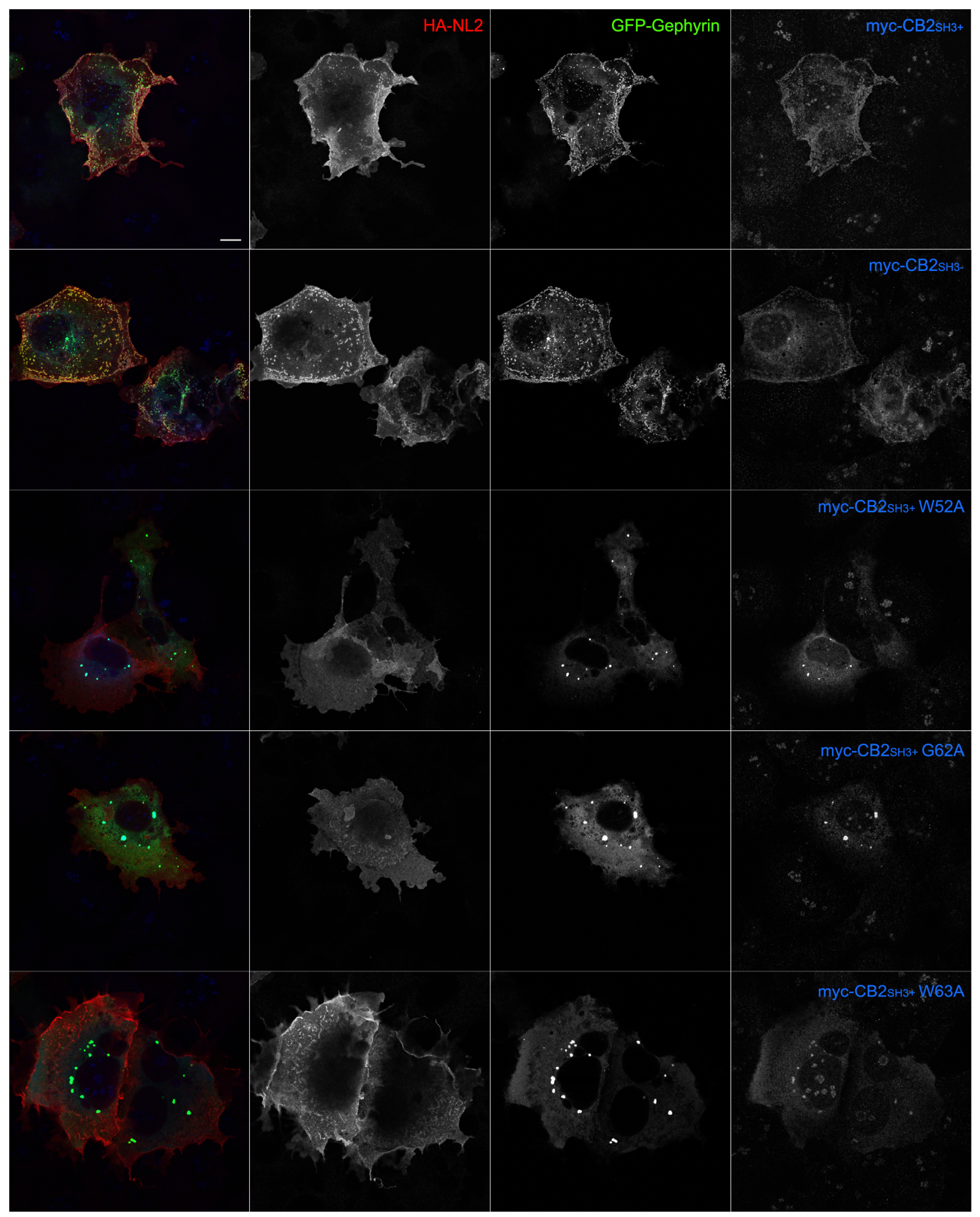


B

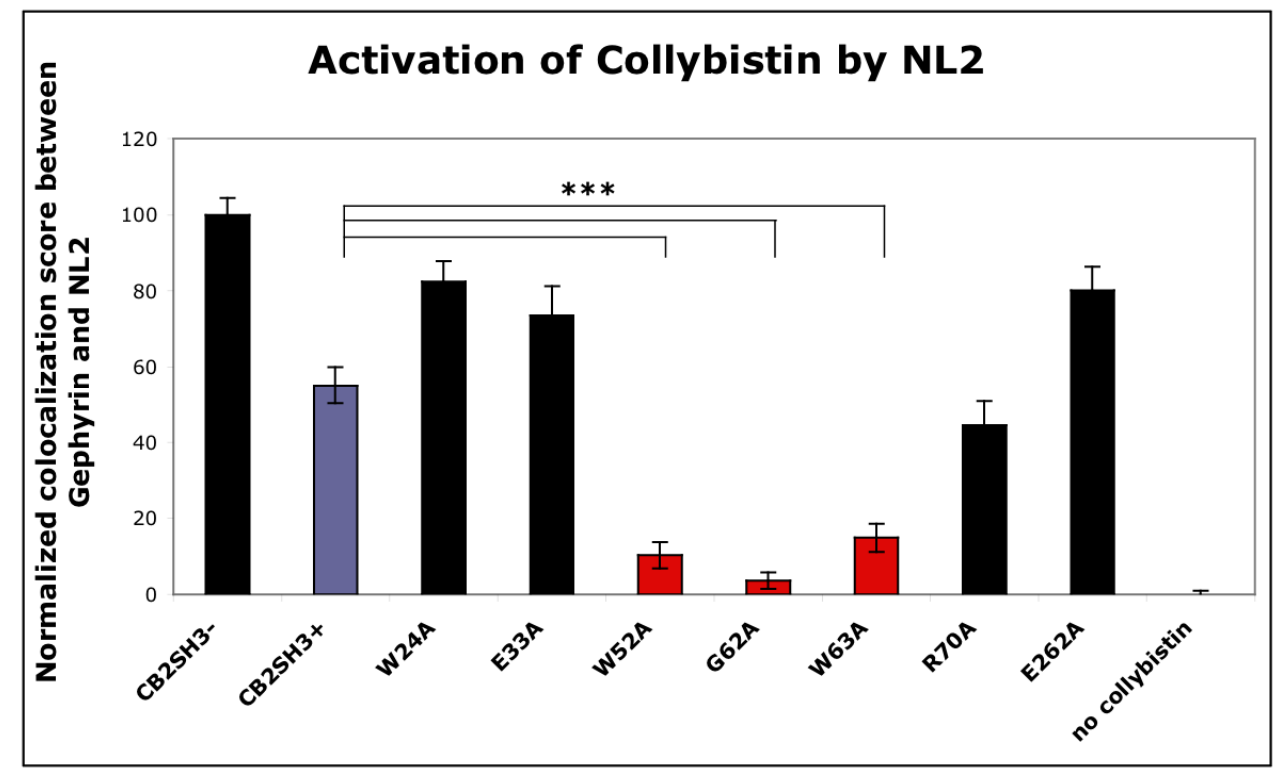

C

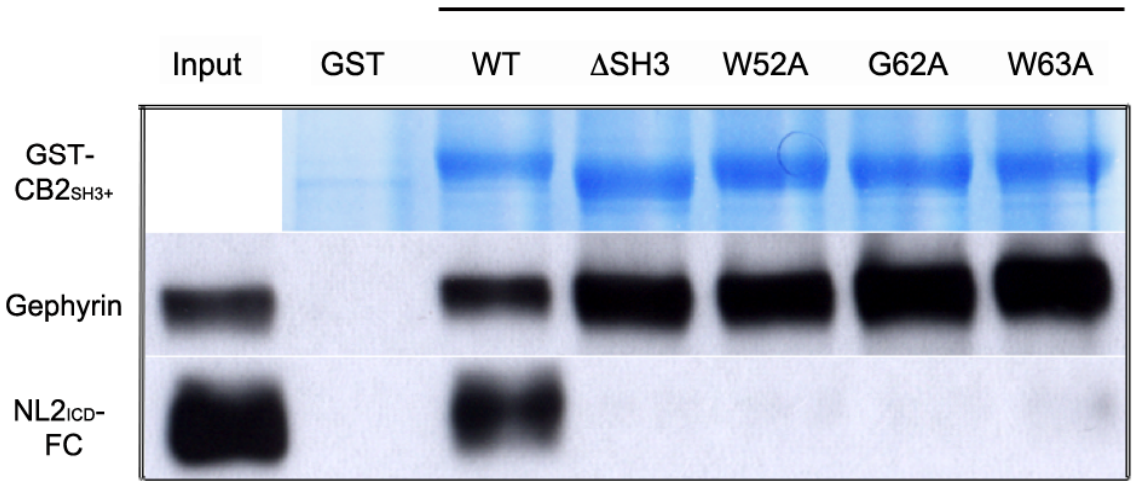

Figure 6: NL2-mediated activation of Collybistin depends on the NL2-Collybistin interaction (A) COS7 cells were transfected with HA-NL2 (red), GFP-Gephyrin (Green) and myc-CB2 $2_{\mathrm{SH}_{3}+}$ its point mutants or myc-CB2 ${ }_{\mathrm{SH} 3-}$. myc-CB2 $2_{\mathrm{SH} 3+}$ constructs bearing the W52A, G62A and W63A mutations were incapable of being activated by NL2 and redistributing Gephyrin to submembrane microclusters. Scale bar: $20 \mu \mathrm{m}$. (B) The correlation coefficients of GFP- and surface stained HA- signals of GFP-Gephyrin and HA-NL2, respectively, were measured to quantify the level of NL2-induced activation of each Collybistin mutant. Correlation coefficient values were normalized to the constitutively active Collybistin variant $\left(\mathrm{CB}_{\mathrm{SH}_{3}-}\right)$, representing $100 \%$ activation, and baselined to samples lacking Collybistin, representing $0 \%$ activation. Compared to $\mathrm{CB} 2_{\mathrm{SH}_{3+}}$ the mutants $\mathrm{W} 52 \mathrm{~A}, \mathrm{G} 62 \mathrm{~A}$ and $\mathrm{W} 63 \mathrm{~A}$ showed a reduced responsiveness to NL2 activation $\left(\mathrm{CB} 2_{\mathrm{SH}_{3}}: 55.19 \% \pm 4.86, \mathrm{~N}=3, \mathrm{n}=20 ; \mathrm{W} 52 \mathrm{~A}: 10.30 \% \pm 3.49, \mathrm{~N}=3, \mathrm{n}=15\right.$, $\mathrm{P}<0.001 ; \mathrm{G} 62 \mathrm{~A}: 3.66 \% \pm 2.15, \mathrm{~N}=3, \mathrm{n}=15, \mathrm{P}<0.001 ; \mathrm{W63A}: 14.88 \% \pm 3.73, \mathrm{~N}=3, \mathrm{n}=15$, $\mathrm{P}<0.001$ ). (C) Using GST-pulldown assays, Full-length GST-CB2 ${ }_{\mathrm{SH} 3+} \mathrm{SH} 3$ domain lacking mutant

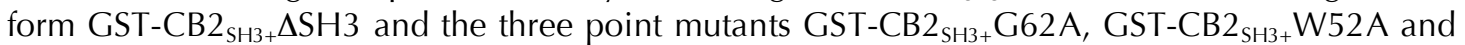
GST-CB2 ${ }_{\mathrm{SH}_{3}}$ W63A were tested for binding to endogenously expressed Gephyrin from HEK cells and exogenously expressed NL2 cytoplasmic domain. GST-CB2 ${ }_{\mathrm{SH} 3}{ }_{+} \Delta \mathrm{SH} 3$ and all the point mutants of GST-CB2 $2_{\mathrm{SH} 3}$, were deficient in binding to $\mathrm{NL2}$, while they all retained their ability to interact with Gephyrin. 
Collybistin variant $\left(\mathrm{CB}_{\mathrm{SH}_{3}-}\right)$ to the score of $100 \%$, and the samples lacking Collybistin represent $0 \%$ activation. Compared to $\mathrm{CB} 2_{\mathrm{SH}_{3+}}$ the mutants W52A, G62A and W63A showed a reduced responsiveness to NL2 activation $\left(\mathrm{CB} 2_{\mathrm{SH}_{3}}\right.$ : $55.19 \% \pm 4.86, \mathrm{~N}=3, \mathrm{n}=20 ; \mathrm{W} 52 \mathrm{~A}: 10.30 \% \pm 3.49, \mathrm{~N}=3, \mathrm{n}=15, \mathrm{P}<0.001$; G62A: $3.66 \% \pm 2.15, N=3, n=15, P<0.001 ; W 63 A: 14.88 \% \pm 3.73, N=3, n=15$, $\mathrm{P}<0.001)$. This set of data indicate that altering the putative NL2-binding site on the $\mathrm{SH} 3$ domain renders Collybistin irresponsive to NL2 induced activation, and that the interaction between NL2 and the SH3 domain of Collybistin is essential for the subsequent membrane targeting of the Gephyrin/Collybistin complex.

In order to obtain biochemical evidence of the effects of point mutants W52A, G62A and W63A on NL2 binding, a mutagenesis screen using GST-pulldown was performed (Figure 6C). Full-length GST-CB2 $2_{\mathrm{SH}_{3+}}$ the $\mathrm{SH} 3$ domain lacking mutant form GST-CB2 ${ }_{\mathrm{SH}_{3}} \Delta \mathrm{SH} 3$ and the three point mutants GST-CB2 ${ }_{\mathrm{SH}_{3}+} \mathrm{G} 62 \mathrm{~A}$, GST-CB2 $2_{\mathrm{SH}_{3}}$ W52A and GST-CB2 ${ }_{\mathrm{SH}_{3}}$ W63A were tested for binding to endogenously expressed Gephyrin from HEK cells and exogenously expressed NL2 ICD fused to an Fc-tag. All GST proteins except GST tag alone bind to Gephyrin to a similar extent, but the $\Delta \mathrm{SH} 3$ mutant and all the point mutants of Collybistin tested in this assay entirely lost their ability to bind NL2 ICD (Figure 6C). Correlating these biochemical data with the cell-biological readouts shown in Figures 5 and 6 indicates that the lack of responsiveness of mutants G62A, W52A and W63A to the presence of NL2 is due to the loss of affinity of Collybistin mutants to the NL2 cytoplasmic tail.

The cell based mutagenesis assay indicates that Collybistin may act as a molecular switch, which undergoes transition between open and closed conformations. In its cytoplasmic state, through an intramolecular interface formed between the $\mathrm{SH} 3$ and the $\mathrm{DH} / \mathrm{PH}$ domains, Collybistin is locked in a closed state where it is inactive and deficient in targeting Gephyrin to the plasma membrane. Binding of the NL2 cytoplasmic tail to the Collybistin SH3 domain induces a conformational change in Collybistin towards an open state where the inhibiting effect of the $\mathrm{SH} 3$ domain on membrane targeting is 
alleviated, resulting in the tethering of the Gephyrin scaffold to the plasma membrane via the interaction of the $\mathrm{PH}$ domain with membrane lipids.

A
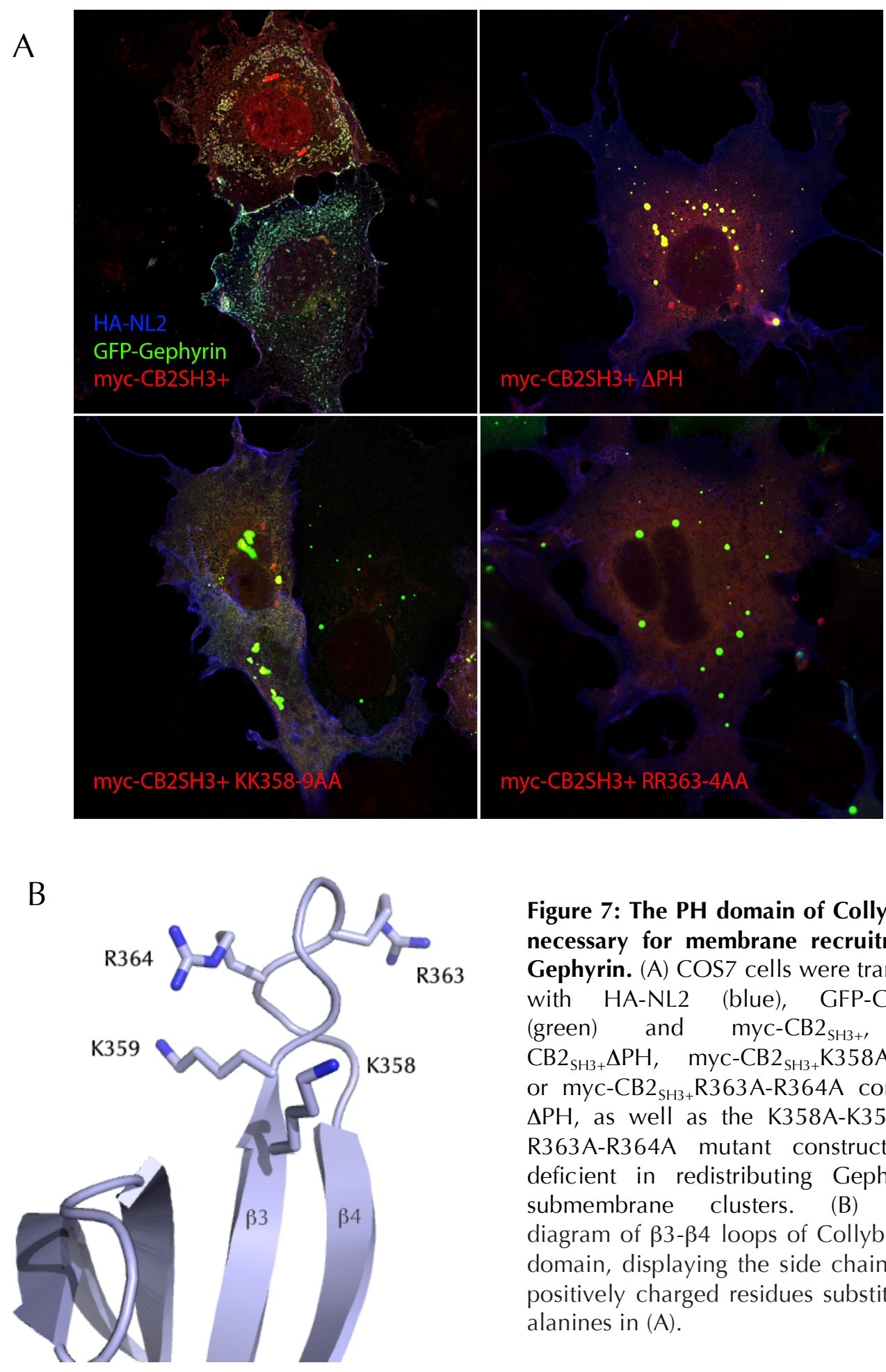

Figure 7: The PH domain of Collybistin is necessary for membrane recruitment of Gephyrin. (A) COS7 cells were transfected with HA-NL2 (blue), GFP-Gephyrin (green) and myc-CB2 $2_{\mathrm{SH} 3+1}$ myc$\mathrm{CB} 2{ }_{\mathrm{SH} 3+} \Delta \mathrm{PH}, \quad$ myc-CB2 ${ }_{\mathrm{SH}_{3}} \mathrm{~K} 358 \mathrm{~A}-\mathrm{K} 359 \mathrm{~A}$ or myc-CB2 ${ }_{\mathrm{SH} 3+} \mathrm{R} 363 \mathrm{~A}-\mathrm{R} 364 \mathrm{~A}$ constructs. $\triangle \mathrm{PH}$, as well as the K358A-K359A and R363A-R364A mutant constructs were deficient in redistributing Gephyrin to submembrane clusters. (B) Ribbon diagram of $\beta 3-\beta 4$ loops of Collybistin $\mathrm{PH}$ domain, displaying the side chains of the positively charged residues substituted by alanines in $(\mathrm{A})$. 
How does the putative intramolecular interaction between the $\mathrm{SH} 3$ and the $\mathrm{DH} / \mathrm{PH}$ domains inhibit the synaptic function of Collybistin? In close homologues of Collybistin, where the $\mathrm{SH} 3$ and $\mathrm{DH} / \mathrm{PH}$ domains form an extensive interface, such as Asef and Intersectin, the $\mathrm{SH} 3$ domain is responsible for inhibiting the GEF activity of the respective DH domain (Murayama et al., 2007; Mitin et al., 2007; Ahmad et al., 2010). In fact, it has been shown that compared to $\mathrm{CB}_{\mathrm{SH}_{3+}} \mathrm{CB} 2_{\mathrm{SH}_{3}-}$ is a better catalyst for guanine exchange of Cdc42 (Xiang et al., 2006). However, a direct link between the enzymatic activity of the $\mathrm{DH}$ domain and the role of Collybistin in Gephyrin clustering still remains to be identified. On the contrary, it has been shown that a $\mathrm{CB} 2_{\mathrm{SH}_{3}-}$ mutant lacking GEF activity towards Cdc42 was still fully functional in deploying the Gephyrin scaffold both in neuronal and in non-neuronal cells (Reddy-Alla et al., 2010). Given that the interface formed between $\mathrm{SH} 3$ and $\mathrm{DH} / \mathrm{PH}$ domains involves a significantly large portion of the $\mathrm{PH}$ domain as well, it could be speculated that the $\mathrm{SH} 3$ domain might inhibit the interaction of the $\mathrm{PH}$ domain with membrane lipids (§ 3.1.10).

\subsubsection{An intact $\mathrm{PH}$ domain is necessary for NL2-induced membrane targeting of Collybistin}

The critical role of the $\mathrm{PH}$ domain in targeting Gephyrin to the plasma membrane in non-neuronal cells and formation of Gephyrin scaffold in cultured neurons has been well documented. Deleting the whole $\mathrm{PH}$ domain in constitutively active $\mathrm{CB} 2_{\mathrm{SH}_{3}-}$ background caused Collybistin to be retained in cytoplasmic blobs and drastically reduced the membrane recruitment of the Gephyrin/Collybistin complex (Harvey et al., 2004, Kalscheuer et al., 2009, Reddy-Alla et al., 2010). It would be interesting to see if deleting the $\mathrm{PH}$ domain in the $\mathrm{CB} 2_{\mathrm{SH}_{3}+}$ background and coexpressing this construct along with Gephyrin and NL2 would have a similar consequence. Indeed, myc-CB2 ${ }_{\mathrm{SH}_{3}+} \mathrm{APH}$ was unable to target Gephyrin to the plasma membrane in the presence of NL2, indicating that membrane tethering of the Gephyrin scaffold requires a 
functional $\mathrm{PH}$ domain to mediate membrane tethering of the Gephyrin/Collybistin complex (Figure 7). However, since the $\mathrm{PH}$ domain is a large unit of Collybistin that may have additional roles in the structural integrity of the protein, e.g. by assisting the $\mathrm{DH}$ domain in its enzymatic activity or by binding to other accessory proteins, deleting the $\mathrm{PH}$ domain may have caused pleiotropic effects on Collybistin function. Therefore, the primary sequence of the $\mathrm{PH}$ domain was screened for single amino acids that might mediate the interaction of the $\mathrm{PH}$ domain with membrane lipids. It has been shown that the $\beta 3 / \beta 4$ loop of the $\mathrm{PH}$ domain contains basic amino acids that bind to membrane phosphoinositides (Hyvönen et al., 1995; Yu et al., 2004; DiNitto \& Lambright, 2006). The $\beta 3 / \beta 4$ loop of the Collybistin $\mathrm{PH}$ domain also carries several lysine and arginine residues, which may be responsible for mediating the interaction between the $\mathrm{PH}$ domain and the specific phosphoinositides that Collybistin may bind to. Replacement of two adjacent lysines (K358/K359) and two adjacent arginines (R363/R364) with alanines resulted in loss-of-function of the $\mathrm{PH}$ domain and intracytoplasmic accumulation of Collybistin, tightly attached to Gephyrin aggregates (Figure 7). These results show that phosphoinositide binding is an essential step for attachment of Gephyrin/Collybistin complex to plasma membrane sites.

\subsubsection{The SH3 domain inhibits binding of Collybistin to PI3P}

Although $\mathrm{PH}$ domains are found in a large variety of protein families and are highly conserved in sequence and structure, it is difficult to deduce information regarding the phosphoinositide specificity of a given $\mathrm{PH}$ domain by making sequence alignments to previously characterized $\mathrm{PH}$ domains. It has been shown that the constitutively active $\mathrm{CB} 2_{\mathrm{SH}_{3}-}$ binds specifically to phosphatidlylinositol-3-phosphate (PI3P) (Kalscheuer et al., 2009; Reddy-Alla et al., 2010), a phosphoinositide derivative with an emerging role in membrane trafficking and signal transduction. In the current study, $\mathrm{CB} 2_{\mathrm{SH} 3+}$ and its domain deletion mutants $\left(\mathrm{CB} 2_{\mathrm{SH}_{3}+} \Delta \mathrm{SH} 3\right.$ and $\left.\mathrm{CB} 2_{\mathrm{SH}_{3}+} \Delta \mathrm{PH}\right)$ were recombinantly expressed with a GST-tag and tested for phosphoinositide binding by protein lipid overlay assays, 
using commercially available membrane strips (Echelon ${ }^{\mathrm{TM}}$ ). $\mathrm{CB} 2_{\mathrm{SH}_{3}+} \Delta \mathrm{SH} 3$ (analogous to constitutively active $\mathrm{CB} 2_{\mathrm{SH}_{3} \text { ) }}$ exhibited robust binding to $\mathrm{PI} 3 \mathrm{P}$ and modest binding to PI4P (Figure 8B). Interestingly, and in contrast to GST-CB2 ${ }_{\mathrm{SH} 3}$ GST-CB2 $2_{\mathrm{SH}_{3}+}$ did not bind to PI3P; it rather showed low and promiscuous binding to a broad range of phosphoinositide derivates. As expected, complete deletion of the $\mathrm{PH}$ domain caused complete loss of lipid binding, as demonstrated by GST-CB2 ${ }_{\mathrm{SH} 3+} \Delta \mathrm{PH}$.

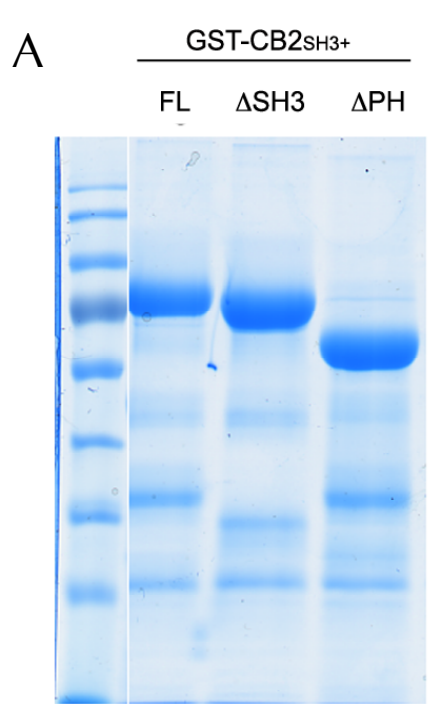

Figure 8: Collybistin specifically binds to Phosphatidylinositol-3-phosphate (PI3P) via its PH domain: (A) SDS-PAGE and Coomassie staining of glutathione affinity purified samples of bacterially expressed GST-CB2 $2_{\mathrm{SH} 3+}$ GST-CB2 $2_{\mathrm{SH} 3+} \Delta \mathrm{SH} 3$ and GST-CB2 ${ }_{\mathrm{SH} 3+} \Delta \mathrm{PH}$. (B) Protein-lipid overlay assays were employed using the commercially available PIP-Strips. Bound proteins were detected using an anti-GST antibody. GST-CB2 ${ }_{\mathrm{SH} 3+} \Delta \mathrm{SH} 3$ specifically interacts with phosphatidylinositol-3-phosphate (PI3P). GST-CB2 $2_{\mathrm{SH} 3+}$ and GST-CB2 $2_{\mathrm{SH} 3+} \Delta \mathrm{PH}$ do not selectively bind to any lipid derivative. GST tagged $\mathrm{PH}$ domain of PLC81, which binds specifically to phosphatidyl-inositol-4,5-bisphosphate $\left(\mathrm{PI}(4,5) \mathrm{P}_{2}\right)$ was used as a positive control to determine the reliability of the assay. (C) Custom-made PI3P strips were used to compare different Collybistin mutants for PI3P binding. Bound proteins were detected using an anti-GST antibody. GST$\mathrm{CB}_{\mathrm{SH}_{3}+} \Delta \mathrm{SH} 3$ and GST-CB2 $2_{\mathrm{SH}_{3}+} \mathrm{E} 262 \mathrm{~A}$ were capable of binding to PI3P, whereas GST-CB2 $2_{\mathrm{SH} 3+1}$ GST-CB2 $2_{\mathrm{H} 3+} \Delta \mathrm{PH}$ and GST-CB2 $2_{\mathrm{SH} 3+} \Delta \mathrm{SH} 3-\mathrm{R} 363 \mathrm{~A}-$ R364A displayed no affinity. 
The clear difference between the PI3P binding capabilities of $\mathrm{CB}_{2 \mathrm{SH}_{3}} \Delta \mathrm{SH} 3$ and $\mathrm{CB} 2_{\mathrm{SH}_{3}+}$ indicated that the presence of the $\mathrm{SH} 3$ domain might indeed obstruct the membrane tethering of the $\mathrm{PH}$ domain, rendering $\mathrm{CB}_{\mathrm{SH}_{3}+}$ an inactive Collybistin variant, which requires an activation step mediated by NL2 or NL4. In the absence of the $\mathrm{SH} 3$ domain, no such activation is required, as was shown previously, and thus $\mathrm{CB} 2_{\mathrm{SH}_{3} \text { - }}$ acts as a constitutively active Collybistin variant that can autonomously tether the Gephyrin scaffold to the plasma membrane.

To further elucidate the mechanism of lipid binding, PI3P strips were generated by spotting $\mathrm{PI} 3 \mathrm{P}$ in varying concentrations onto a nitrocellulose membrane (Figure 8C). Once again, it was clearly demonstrated that GST-CB2 ${ }_{\mathrm{SH}_{3}+} \Delta \mathrm{SH} 3$ has the highest affinity for PI3P, while GST-CB2 ${ }_{\mathrm{SH} 3+}$ shows no detectable binding. Interestingly, a point mutation in the DH domain, E262A, showed increased affinity for PI3P. This mutant was previously shown to render Collybistin constitutively active in the cell-based assays by abolishing the putative intramolecular interaction between the $\mathrm{SH} 3$ and $\mathrm{DH} / \mathrm{PH}$ domains (Figure 5). These data support the idea that the $\mathrm{SH} 3$ domain may prevent the $\mathrm{PH}$ domain $\mathrm{PI3P}$ interaction through steric hindrance as it folds onto the $\mathrm{DH} / \mathrm{PH}$ interface. Disrupting the association of the $\mathrm{SH} 3$ with the $\mathrm{DH}$ and $\mathrm{PH}$ domains by the E262A substitution disengages the $\mathrm{SH} 3$ domain and gives the $\mathrm{PH}$ domain unobstructed access to membrane lipids.

The lipid overlay assays also served as a useful tool to study the functional residues in the $\mathrm{PH}$ domain required for binding to $\mathrm{PI} \mathrm{P}$, and corroborate data on $\mathrm{PH}$ domain mutants in cell-based assays. The double-arginine mutation (R363AR364A), which caused Collybistin to be deficient in targeting Gephyrin to the plasma membrane in the presence of NL2 in the cell based assay, this time was introduced in the constitutively active GST-CB2 ${ }_{\mathrm{SH} 3+} \Delta \mathrm{SH} 3$ background. Indeed, this mutant construct does not to bind to PI3P, confirming both the critical role of residues R363 and R364 and the link between plasma membrane targeting of Collybistin complexes and PI3P binding of the Collybistin PH domain. 
A

\begin{tabular}{|c|c|c|c|c|c|c|c|c|c|}
\hline & \multicolumn{3}{|c|}{ GST-CB2SH3+ } & \multicolumn{3}{|c|}{$\mathrm{GST}-\mathrm{CB} 2 \mathrm{SH} 3+\triangle \mathrm{SH} 3$} & \multicolumn{3}{|c|}{ GST-CB2SH3+ $\Delta \mathrm{PH}$} \\
\hline [NL2cyt] & NL2cyt & PI3P & $\begin{array}{l}\text { NL2cyt } \\
\text { PI3P }\end{array}$ & NL2cyt & PI3P & $\begin{array}{c}\text { NL2cyt } \\
+ \\
\text { PI3P }\end{array}$ & NL2cyt & PI3P & $\begin{array}{c}\text { NL2cyt } \\
+ \\
\text { PI3P }\end{array}$ \\
\hline $750 \mathrm{ng}$ & & e & - & & . & () & & 6 & $s$ \\
\hline $375 \mathrm{ng}$ & & e & 0 & & 8 & () & & 항 & 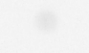 \\
\hline $150 \mathrm{ng}$ & & 6 & $\bullet$ & & $\mathbf{P}$ & (6) & & 9 & $=$ \\
\hline Ong & & e & e & & - & e & & & \\
\hline
\end{tabular}

B
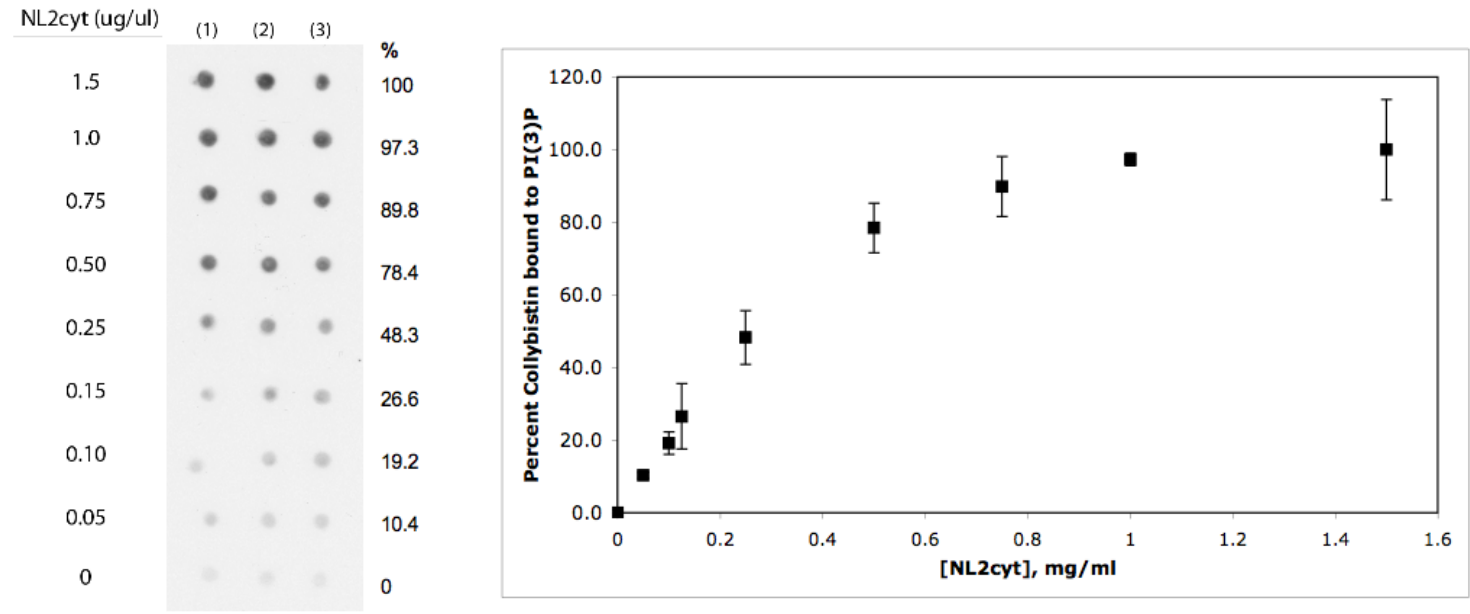

Figure 9: NL2 enhances the lipid binding capacity of $\mathbf{C B 2}_{\mathrm{SH}_{3+}}$ (A) Varying amounts of recombinant NL2 cytoplasmic domain (750 - $375-150-0 \mathrm{ng})$ and constant amount of PI3P $(1 \mu \mathrm{g})$ were spotted on the exact same position of the membrane strips. Bound proteins were detected using an anti-GST antibody. Binding of GST-CB2 $2_{\mathrm{SH} 3+}$ to PI3P was stronger on the strips where NL2 and PI3P were spotted together, compared to the strip where PI3P was spotted alone. Induction of $\mathrm{PI} 3 \mathrm{P}$ binding by NL2 was not detected for GST-CB2 ${ }_{\mathrm{SH} 3+} \Delta \mathrm{SH} 3$ or for GST$\mathrm{CB} 2_{\mathrm{SH} 3+} \Delta \mathrm{PH}$. (B) The effect of NL2 on PI3P binding of Collybistin was assessed quantitatively by

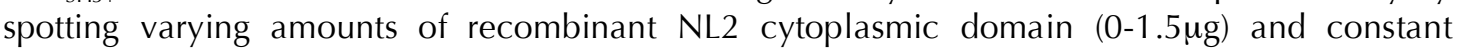
amount of PI3P $(0.5 \mu \mathrm{g})$ on the same position of membrane strips. Bound GST-CB2 ${ }_{\mathrm{SH} 3+}$ on the $\mathrm{PI} 3 \mathrm{P} / \mathrm{NL} 2$ spots was detected using an anti-GST antibody. The induction of NL2 on PI3P binding of Collybistin was assessed by normalizing the amount of protein bound to the spots containing $1.5 \mu \mathrm{g}$ of NL2 to $100 \%$ and the spots lacking NL2 to $\%$. Note that the amount of bound GST$\mathrm{CB} 2_{\mathrm{SH} 3+}$ on the PI3P spots is dependent on NL2 concentration. Plotting the normalized intensity of bound GST-CB2 $2_{\mathrm{SH} 3+}$ for each spot yielded a sigmoidal curve, representing allosteric effect of NL2 on the PI3P binding capacity of Collybistin. 


\subsubsection{NL2 leads to enhanced PI3P binding of Collybistin}

Data from the cell-based structure-function analysis (Figures 5-6) and the protein lipid overlay assays (Figure 8) point towards a molecular switch mechanism. In its free state, Collybistin is in a closed conformation where the SH3 domain obstructs $\mathrm{PH}$ domain access to membrane phosphoinositides. In its NL2-bound state, a conformational change towards a more extended conformation takes place, alleviating the inhibitory effect of the $\mathrm{SH} 3$ domain by pulling it away from the $\mathrm{DH} / \mathrm{PH}$ domains so that the $\mathrm{PH}$ domain may gain access to membrane PI3P. To test the validity of this model, the effect of NL2 on the capacity of Collybistin to bind lipids was examined in vitro (Figure 9A). NL2-PI3P strips were prepared according to the following plan: For each protein to be tested (GST-CB2 $2_{\mathrm{SH}_{3+}}$ GST-CB2 $2_{\mathrm{SH}_{3}+} \Delta \mathrm{SH} 3$, GST-CB2 ${ }_{\mathrm{SH} 3+} \Delta \mathrm{PH}$ ), three different strips were prepared. The first set of strips was spotted only with varying amounts of recombinant NL2 cytoplasmic tail $(750,375,150,0$ ng). The second strip contained constant amounts of PI3P (1 $\mu \mathrm{g}$ on each spot). The third strip contained a mixture of PI3P and NL2 spotted together, with a constant amount of PI3P $(1 \mu \mathrm{g})$ and varying amounts of NL2. Each strip was incubated with $2 \mu \mathrm{g}$ of GST- fusion protein. It should be noted that the binding of GST-CB2 ${ }_{\mathrm{SH}_{3}+}$ or GST-CB2 ${ }_{\mathrm{SH}_{3}+} \Delta \mathrm{PH}$ to NL2 alone was negligible. In line with previous observations, GST-CB2 ${ }_{\mathrm{SH} 3+}$ binds to PI3P with relatively lower affinity than GST$\mathrm{CB} 2_{\mathrm{SH}_{3}} \Delta \mathrm{SH} 3$. However binding of GST-CB2 $2_{\mathrm{SH} 3+}$ to PI3P was stronger on the strips where NL2 and PI3P were spotted together, compared to the strip where PI3P was spotted alone. Induction of PI3P binding by NL2 was not detected for GST-CB2 ${ }_{\mathrm{SH}_{3}} \Delta \mathrm{SH} 3$ or for GST-CB2 ${ }_{\mathrm{SH} 3+} \Delta \mathrm{PH}$.

To determine the effect of NL2 on the PI3P binding of Collybistin quantitatively, a new NL2 / PI3P strip was prepared where 500 ng of PI3P spotted together with varying amounts of recombinant NL2 cytoplasmic tail, ranging from $1.5 \mu \mathrm{g}$ to 0 $\mu \mathrm{g}$ (Figure 9B). By measuring the intensity of bound GST-CB2 ${ }_{\mathrm{SH}_{3}+}$ on each spot, a titration curve was obtained, where the spot with $1.5 \mu \mathrm{g}$ of NL2 and the spot where no NL2 is present were normalized to $100 \%$ and $0 \%$, respectively. It 
could be noted that the effect of NL2 on the PI3P binding of Collybistin is dosedependent and saturable, resulting in a sigmoidal curve, demonstrating the allosteric nature of the NL2 induced PI3P binding of Collybistin.
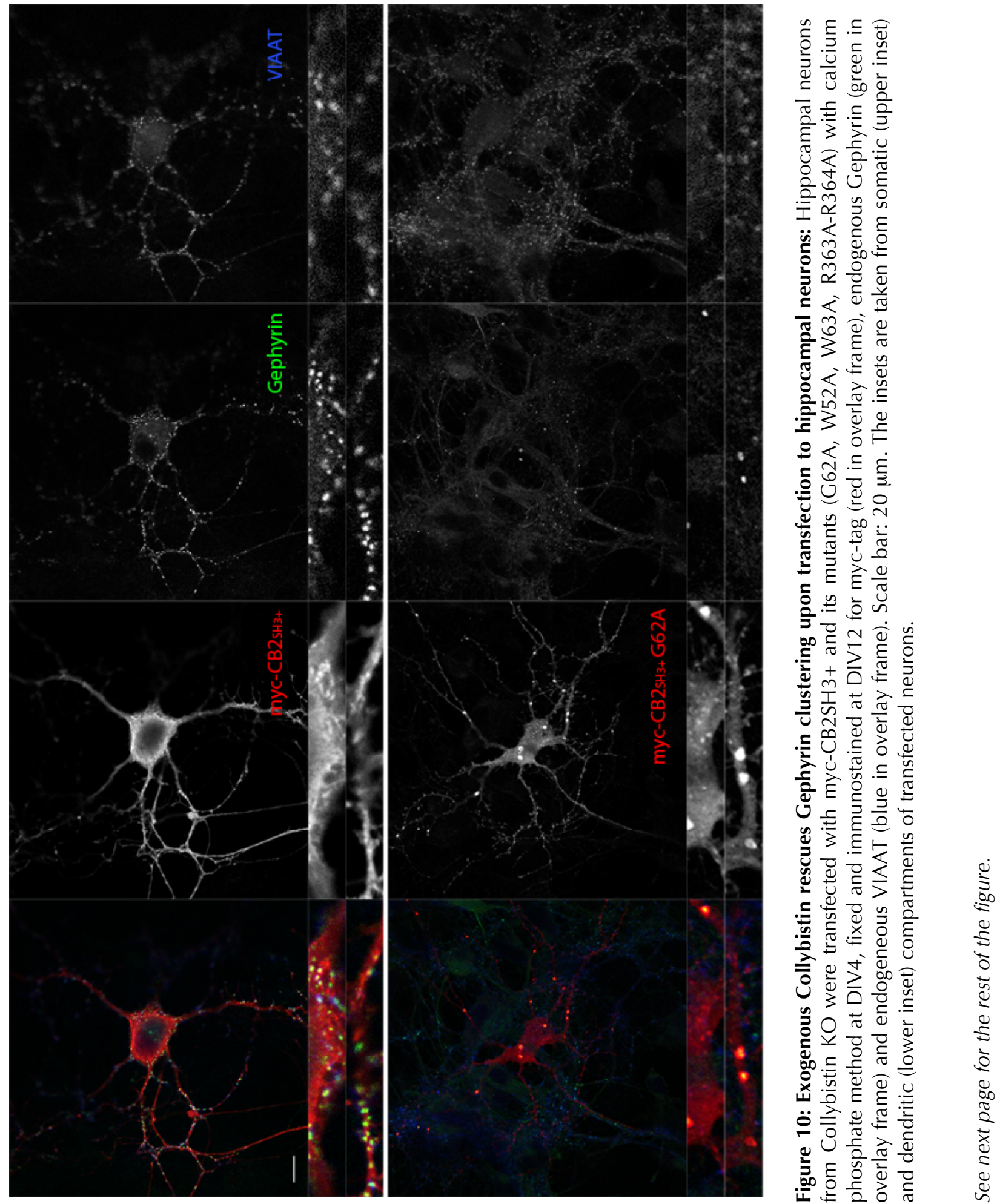


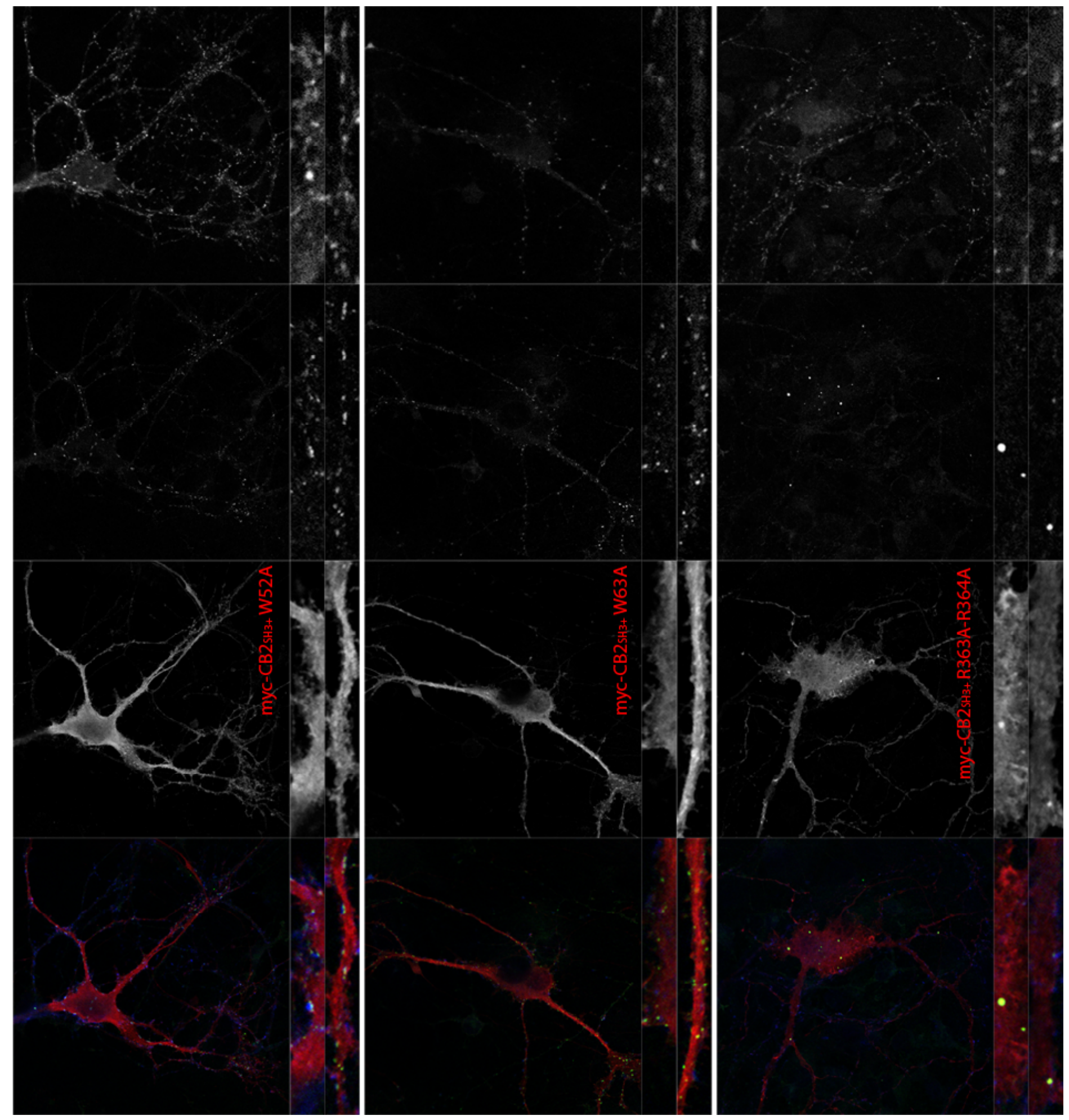




\subsubsection{Expression of exogenous $\mathrm{CB} 2_{\mathrm{SH}_{3}}$ in Collybistin $\mathrm{KO}$ neurons rescues Gephyrin clustering}

In order to elucidate the mechanism of NL2 induced activation of Collybistin in a neuronal context, a mutagenesis approach, similar to the cell based assays, was performed in hippocampal neurons prepared from Collybistin KO PO mice (Figure 10). As shown before, the loss of Collybistin causes drastic deficits in synaptic clustering of Gephyrin in various brain regions both in the perisomatic and in the dendritic areas of the neurons (Papadopoulos et al., 2007). The neurons prepared from Collybistin KO animals were transfected at DIV4 with myc-tagged $\mathrm{CB}_{\mathrm{SH}_{3+}}$ and point mutants of this construct. The neurons were fixed at DIV12 and stained for endogenous Gephyrin and Vesicular Inhibitory Amino Acid Transporter (VIAAT). The neurons transfected with myc-CB2 ${ }_{\mathrm{SH}_{3+}}$ displayed rescued Gephyrin clusters, in good apposition to VIAAT signals, both in the somatic (upper inset) and dendritic (lower inset) compartments. The epilepsycausing G62A mutant of Collybistin gets coclustered with Gephyrin in large, mostly somatic, but occasionally also dendritic, non-synaptic aggregates and displays only extremely low levels of rescue of Gephyrin deposition at the sites marked by VIAAT. Unexpectedly, both of the NL2 binding deficient mutants of Collybistin, W52A and W63A, were able to rescue Gephyrin clustering equally well compared to the WT, both in the perisomatic and also in the dendritic parts. The effects of these mutations might be concealed due to the excessive overexpression and constitutive activation of these constructs, a phenomenon that was also observed in the mammalian cells after around $16 \mathrm{~h}$ of overexpression. In contrast, the PI3P binding deficient R363A/R364A double point mutant of Collybistin displayed inadequate rescue of Gephyrin redistribution to inhibitory synapses, demonstrating that $\mathrm{PI3P}$ binding is necessary for tethering Gephyrin scaffold to the plasma membrane in neurons. 


\subsubsection{Gephyrin-Collybistin interaction revisited: Gephyrin binds to $\mathrm{CB2}{ }_{\mathrm{SH} 3+}$ on multiple sites}

It was shown long ago that both $\mathrm{CB}_{\mathrm{SH}_{3}}$ and $\mathrm{CB} 2_{\mathrm{SH}_{3}-}$ bind to Gephyrin, mediated by a short peptide stretch located N-terminally to the $\mathrm{DH}$ domain. A quadruple alanine replacement of the four charged residues located within this sequence (R107, D108, R111 and E117) resulted in total loss of interaction with Gephyrin (Grosskreutz et al., 2001). However, this finding was disputed later, based on the crystal structure of the $\mathrm{DH} / \mathrm{PH}$ domains of Collybistin. It was claimed that only three out of these four residues are solvent exposed and when these three residues are replaced by alanines in the constitutively active variant of Collybistin lacking the SH3 domain, $\left(\mathrm{CB} 2_{\mathrm{SH} 3-} \mathrm{R} 47 \mathrm{~A} / \mathrm{D} 48 \mathrm{~A} / \mathrm{E} 57 \mathrm{~A}\right)$, there were no deficits in Gephyrin binding (Xiang et al., 2006). Therefore, the exact Gephyrin binding region on Collybistin still remains elusive.

Since there is no other guanine exchange factor or a close homologue of Collybistin that has been identified as an interaction partner of Gephyrin, it could be claimed that the $\mathrm{DH}$ domain of Collybistin must have a specific Gephyrin binding motif that is not conserved in other GEFs. Asef, another GEF belonging to the same family of Cdc42-specific guanine exchange factors as Collybistin, could serve as a useful tool to study the Gephyrin-Collybistin interaction. Asef is the closest homologue of Collybistin in the whole mouse proteome, sharing a similar domain organization and $81 \%$ sequence homology with Collybistin. The major difference between Collybistin and Asef is the Adenomatous polyposis coli (APC) binding region (ABR) of Asef that is upstream of the SH3 domain and absent in Collybistin. By binding to ABR, APC can induce a conformational change in Asef where the $\mathrm{SH} 3$ domain is pulled away from the $\mathrm{DH} / \mathrm{PH}$ interface, followed by the disinhibition of the enzymatic activity of Asef. Due to such high structural and mechanistic similarities between Collybistin and Asef, it was very important to test whether Asef might also be responsible for targeting Gephyrin to the plasma membrane. The cell 


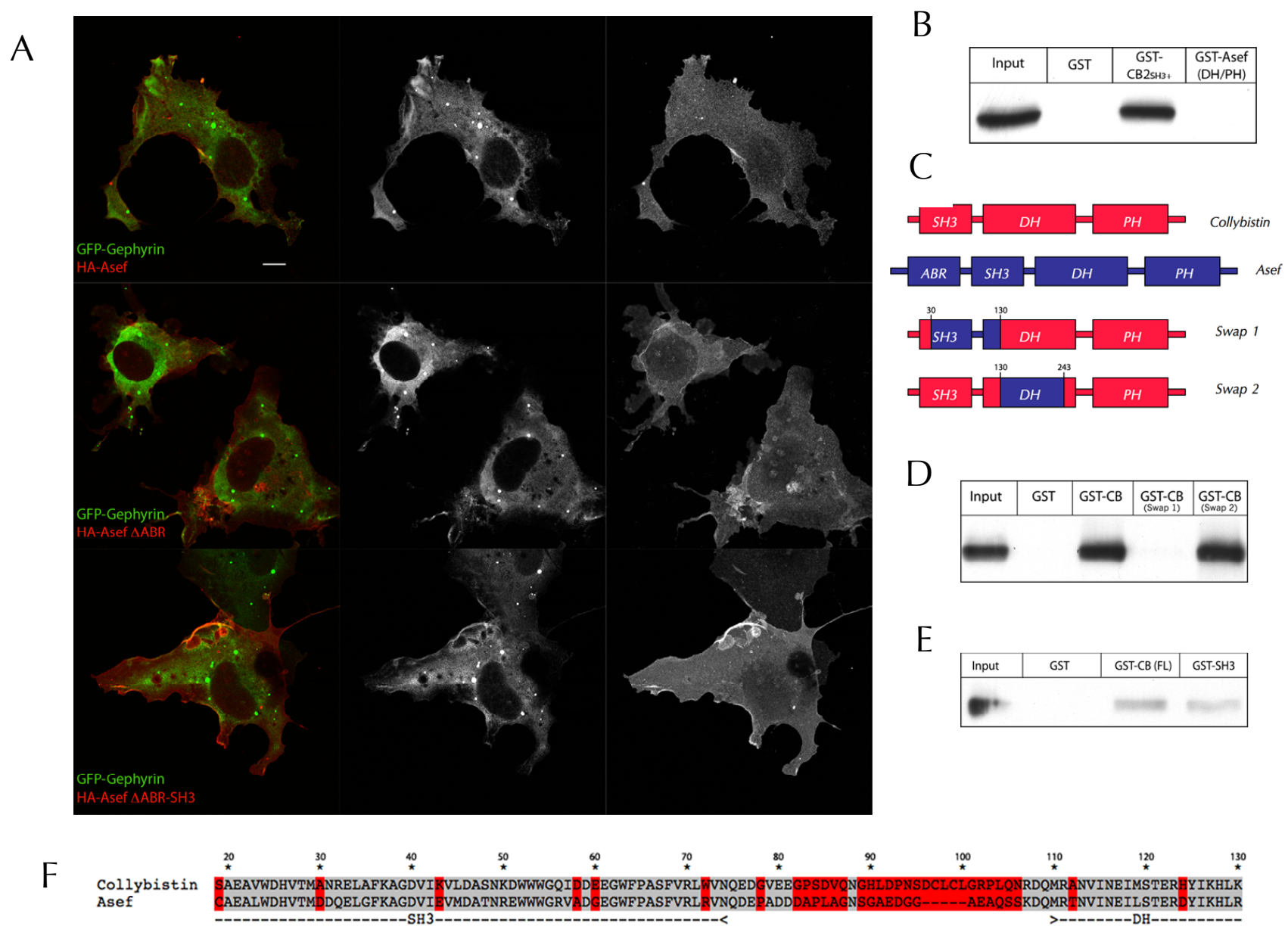

Figure 11: Gephyrin binds to the linker region between $\mathrm{SH} 3$ and $\mathrm{DH}$ domains of Collybistin (A) COS7 cells were cotransfected with HA-Asef and its mutants (HA-Asef $\triangle A B R$ and HA-Asef $\triangle \mathrm{ABR}-\mathrm{SH} 3$ ) (red in overlay frame) and GFP-Gephyrin (green in overlay frame). Asef is not recruited to intracytoplasmic Gephyrin aggregates. (B) GST-pulldowns using GST-CB2 ${ }_{\mathrm{SH} 3+}$ and GST-Asef (DH/PH) constructs to test binding to endogenous Gephyrin produced in HEK293T cells. Asef is unable to bind Gephyrin. (C) Schematic representation of the two chimeric Collybistin-Asef constructs generated by replacing parts of Collybistin (residues between 30130 and 130-243) with homologous parts from Asef, leading to generation of constructs Swap 1 and Swap 2. (D) GST-pulldowns using GST-CB2 ${ }_{\mathrm{SH} 3+}$ and GST-tagged Swap 1 and Swap 2 chimeric constructs. Swap 1 construct is unable to bind Gephyrin, indicating that Gephyrin binding surface on Collybistin resides between residues 30 and 130. (E) GST-pulldowns using GST-CB2 ${ }_{\mathrm{SH} 3+}$ and GST-SH3 constructs to test binding to endogenous Gephyrin produced in HEK293T cells. SH3 domain of Collybistin contains at least one binding site for Gephyrin. (F) Sequence alignment of Collybistin residues between 19 and 130 and homologous portion of Asef. The non-conserved residues are shaded in red.

based assays showed that full length HA-tagged Asef, as well as the Asef mutant lacking the $\mathrm{N}$-terminal $\mathrm{ABR}$ (HA-Asef $\triangle \mathrm{ABR}$; analogous to $\mathrm{CB} 2_{\mathrm{SH}_{3}}$ ) or both the $\mathrm{ABR}$ and the $\mathrm{SH} 3$ domains (HA-Asef $\triangle \mathrm{ABR}-\Delta \mathrm{SH} 3$; analogous to $\mathrm{CB} 2_{\mathrm{SH}_{3}}$ ) were unable to cocluster with Gephyrin, in contrast to Collybistin (Figure 11A). GST- 
pulldown assays using a GST-tagged Asef construct that contains the $\mathrm{DH} / \mathrm{PH}$ domains of Asef (GST-Asef-DH/PH) showed that Asef does not bind to Gephyrin in vitro, unlike Collybistin, which can bind to Gephyrin, both in the absence and in the presence of the SH3 domain (Figure 11B). These observations indicate that despite their structural similarity, Asef cannot replace the synaptic function of Collybistin. Therefore, the Gephyrin binding site on Collybistin is not expected to be conserved in Asef.

To identify the Collybistin-specific region responsible for its specific affinity to Gephyrin, two chimeric constructs were cloned, in which Asef sequences progressively replace respective Collybistin sequences. The first construct covers a large portion of the $\mathrm{SH} 3$ domain and the $\mathrm{N}$-terminal part of the $\mathrm{DH}$ domain (A30-K130; Swap I), and the second one covers the rest of the $\mathrm{DH}$ domain (D131-D243; Swap II) as schematized in Figure 11. These chimeric constructs were GST-tagged and used to examine Gephyrin binding. Interestingly, the Swap I chimeric protein was deficient in Gephyrin binding, while Swap II displayed a similar affinity to Gephyrin as GST-CB2 ${ }_{\mathrm{SH}_{3+}}$ indicating that the Gephyrin binding motifs on Collybistin lie within the 100-residue region covering the $\mathrm{SH} 3$ domain, the linker region and the $\mathrm{DH}$ domain (Figure 11D).

\subsection{Investigation of the effects of serine phosphorylation of Neuroligins on the Gephyrin binding motif}

A stretch of 15 residues in the ICDs of all Neuroligin paralogues has been identified as the Gephyrin binding motif (Poulopoulos et al., 2009; see also Schema 4). This stretch of 15-residues is highly conserved in all paralogues of NLs, however there are minor variations at positions 4, 6 and 10, where serine or threonine residues are replaced by alanines in different paralogues (Fig 12). The phosphorylation profiles of Neuroligin ICDs based on NetPhos 2.0 Server (a server that produces neural network predictions for serine, threonine and tyrosine phosphorylation sites, available at: 
http://www.cbs.dtu.dk/services/NetPhos/) indicates that these serine and threonine residues might be targets of kinases. A serine residue that is conserved among NL1 (S802), NL3 (S799) and NL4 (S880), is particularly interesting because it is replaced by an alanine in NL2 (A777), the only Neuroligin paralogue that is consistently found to be associated with Gephyrin. Therefore, it was postulated that potential phosphorylation of this serine residue in NL1, NL3 and NL4 might negatively affect the Neuroligin-Gephyrin interaction. In fact, the phosphomimetic mutation of the corresponding serine residue in NL1 (S802D) resulted in disruption of the interaction with Gephyrin (A. Poulopoulos, personal communication). A collaborative approach has been conveyed to test whether any of the serine or the threonine residues is phosphorylated in vivo. A mass-spectrometry based proteomics approach revealed that NL3 undergoes phosphorylation at S799 (Box. 1).

To test whether phosphorylation of NL3 at S799 leads to reduced association of NL3 with Gephyrin, a cell based coclustering assay was carried out. COS7 cells were transfected with GFP-Gephyrin and constitutively active myc-CB2 ${ }_{\mathrm{SH} 3-}$ along with HA-NL3 and its phosphodeficient (HA-NL3 S799A) and phosphomimetic (HA-NL3 S799D) mutants. myc-CB2 ${ }_{\mathrm{SH}_{3}-}$ is capable of targeting Gephyrin autonomously to the plasma membrane where microaggregates of Gephyrin/Collybistin cocluster with NL3 (Figure 12). The level of coclustering of each surface stained NL3 variant with the Gephyrin signal was assessed. Compared to HA-NL3 and the phosphodeficient HA-NL3 S799A, the phosphomimetic S799D mutant displayed reduced coclustering with membrane targeted Gephyrin (Pearson's correlation coefficient for HA-NL3: 0.595 \pm 0.112 ; HA-NL3 S799A: 0.571 \pm 0.68 ; HA-NL3 S799D: 0.398 $\pm 0.092, N=2 n=10)$. This result indicates that phosphorylation of S799 (or the corresponding S802 in NL1 and S880 in NL4) might in fact negatively regulate the association of Gephyrin with the Neuroligin paralogues that are not consistently localized to inhibitory synapses. 
A

B

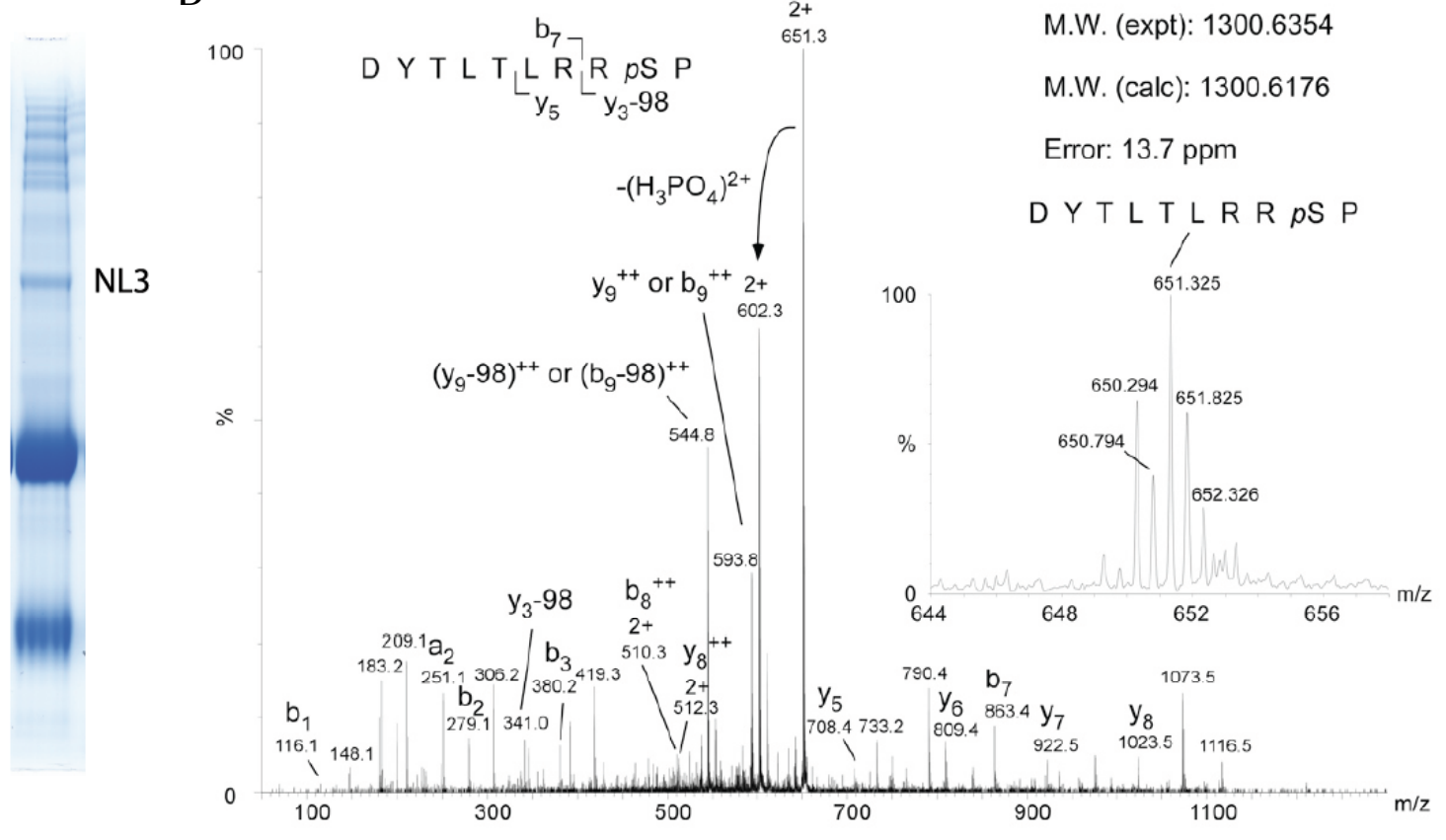

C

$\begin{array}{ll}\text { NL1 } & \text { PDYTLAMRRSPDDIP } \\ \text { NL2 } & \text { PDYTLALRRAPDDVP } \\ \text { NL3 } & \text { PDYTLTLRRSPDIP } \\ \text { NL4 } & \text { PDYALTLRRSPDDVP }\end{array}$

Box 1. Neuroligin 3 is phosphorylated in vivo at Serine 799: (A) NL3 was immunoprecipitated from adult rat brain homogenate using a specific polyclonal NL3 antibody. The immunoprecipitation sample was separated on SDS-PAGE and the Coomassie-stained band containing NL3 was identified by MALDI-TOF/TOF MS/MS mass spectrometry (Brucker Ultraflex). (B) The protein was digested with trypsin in the gel, the resulting peptides were extracted and applied onto a $\mathrm{TiO}_{2}$ column for phospho-peptide enrichment. The phosphopeptide enriched eluate was analyzed on a Q-ToF ESI mass spectrometer (Q-ToF Ultima, Waters). A peptide with molecular mass of 1300.61 (m/z 651.32 for doubly-charged species) matched the mass of a 10-residue peptide from NL3 (between D791-P800) with a single phosphorylation (see inlay spectrum). MS/MS analysis of this peptide showed detection of the $y 5, y 6, y 7, y 8$ and corresponding b2, b3 and b7 ions in their non-phosphorylated form, while the $y 3$ ion with a signature neutral loss of 98 amu suggests that the phosphorylation must be within the last three amino acids of the peptide leaving S799 as the place of phosphorylation. (C) Sequence alignment of the Gephyrin-binding motifs of NL1-4. S799 of NL3 is shaded in red. Note that the phosphorylated serine residue is replaced by an alanine in NL2. 

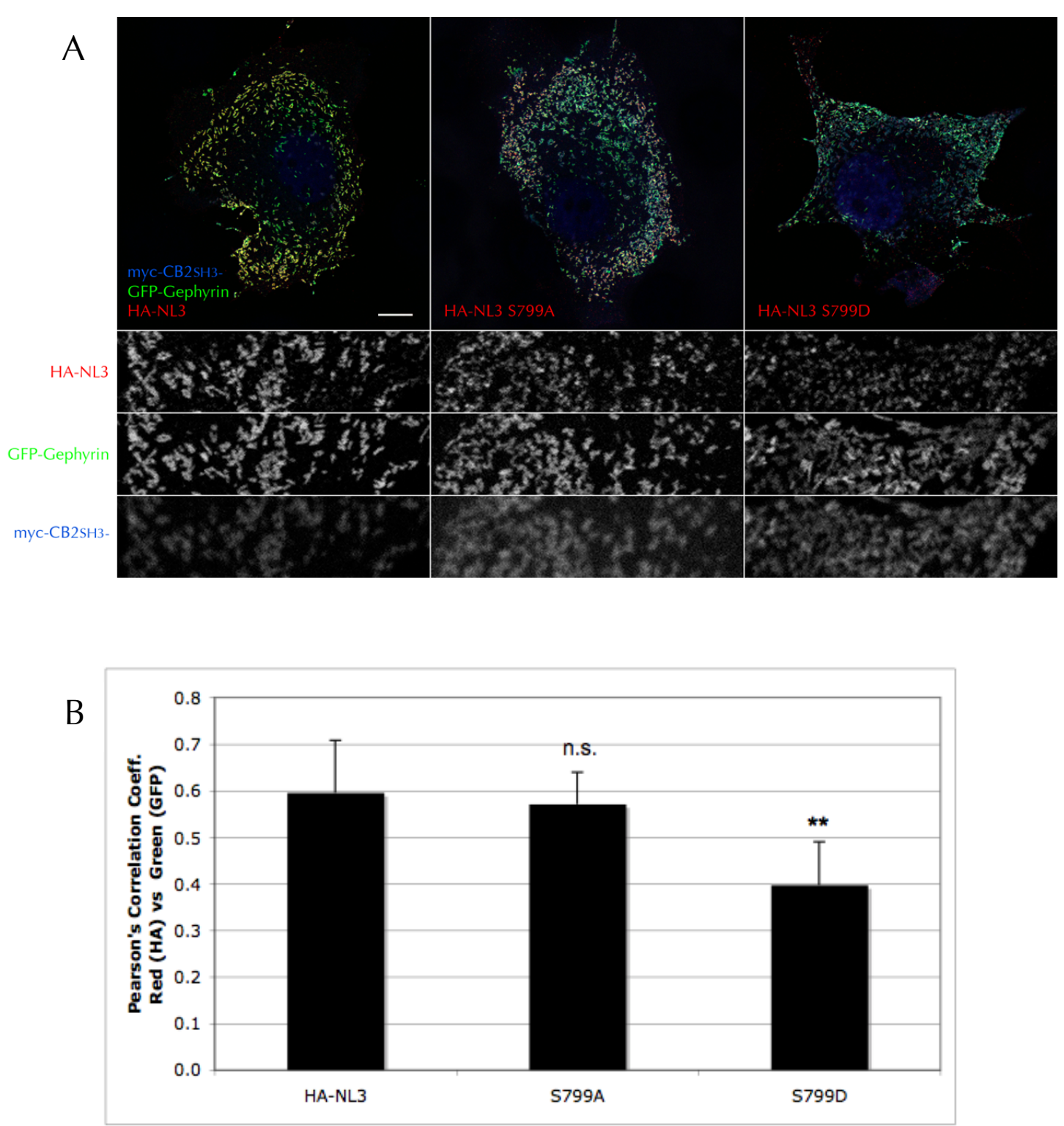

Figure 12: Phosphorylation of Gephyrin-binding motif of NL3 might hinder its association with Gephyrin. (A) COS7 cells were transfected with GFP-Gephyrin (green in overlay image), myc-CB2 $2_{\mathrm{SH} 3}$ (blue in overlay image) and HA-NL3, HA-NL3 S799A (phosphodeficient mutant) or HA-NL3 S799D (phosphomimetic mutant) constructs (red in overlay image). The insets taken from each image display strong colocalization of immunostaining signals for NL3 and S799A mutant with Gephyrin, while the S799D mutation clearly interfered with the association of Gephyrin and NL3. Scale bar: $20 \mu \mathrm{m}$. (B) Pearson's correlation coefficients between HA- and GFP- signals were calculated. HA-NL3: 0.595 \pm 0.112 ; HA-NL3 S799A: $0.571 \pm 0.68$; HA-NL3 S799D: 0.398 $\pm 0.092, \mathrm{~N}=2 \mathrm{n}=10$ for each construct; $\mathrm{P}<0.005$ ). Compared to WT and S799A constructs, HA-NL3 S799D signals exhibited reduced correlation with GFP-Gephyrin signal, indicating that in vivo phosphorylation of NL3 may hinder the association of Gephyrin and NL3. 
To further investigate the functional implications of the serine phosphorylation of Gephyrin-binding motif on NL3 (and potentially on NL1 and NL4) a phosphospecific antibody was raised against a peptide that covers the 15 residue long Gephyrin binding motif with a phosphorylated serine at position 10 (provided by A. Poulopoulos). This antibody was shown to have around $\sim 20$ fold preference for the phosphorylated peptide as compared to its nonphosphorylated variant (A. Poulopoulos, personal communication). Using this antibody to analyse the immunoprecipitated material from the mouse brain, it was possible to show that a significant fraction of NL3 is phosphorylated at S799 (Liam Tuffy, personal communication). It remains to be identified whether NL1 and NL4 are also phosphorylated in vivo. 


\section{DISCUSSION}

In this study, I explored the assembly mechanisms of the postsynaptic apparatus during the formation of inhibitory synapses. There is growing morphological, cell biological, biochemical and genetic evidence that the Neuroligin family of postsynaptic adhesion molecules have substantial roles in synapse assembly, differentiation and maturation (Ichtchenko et al., 1995; Irie et al., 1997; Chih et al., 2005; Varoqueaux et al., 2006; Chubykin et al., 2007; Poulopoulos et al., 2009; Hoon et al., 2011). Characterization of NL2, a Neuroligin paralogue exclusively localized to inhibitory synapses (Varoqueaux et al., 2004) and responsible for inhibitory synapse differentiation (Graf et al., 2004; Poulopoulos et al., 2009), stimulated further research in this field. Subsequent NL2 KO studies substantiated the critical role of NL2 for the maintenance of inhibitory synaptic signaling in different parts of the CNS (Chubykin et al., 2007; Hoon, et al. 2009, Jedlicka et al., 2011). Furthermore, biochemical and cell biological data indicated that NL2 associates with and controls the synaptic accumulation of Gephyrin and subsequent clustering of GABAergic and glycinergic receptors (Poulopoulos et al., 2009). Altogether these studies opened the way for investigating the molecular mechanisms underlying Neuroligin function in the assembly of inhibitory synapses.

\subsection{NL2 as a functional organizer of inhibitory synapse assembly}

According to the current model of inhibitory postsynapse differentiation, NL2 functions as a major molecular determinant of the events that underlie the assembly of the inhibitory postsynaptse apparatus. By directly interacting with Gephyrin and by activating Collybistin, NL2 is capable of demarcating the sites on the plasma membrane where the postsynaptic scaffold is constructed and to which inhibitory neurotransmitter receptors are recruited, in precise apposition 
to inhibitory presynaptic release terminals (Poulopoulos et al., 2009). The mechanistic details of the NL2 mediated signal that allows for Collybistin activation are addressed in the present study.

\subsubsection{Collybistin is a target of NL2 mediated signaling}

Collybistin is a neuron-specific dbl-family guanine nucleotide exchange factor that drives the postsynaptic accumulation of the Gephyrin scaffold. It has been clearly demonstrated that the N-terminal part of Collybistin, which harbors a SH3 domain, is inhibitory to the membrane targeting activity of this protein. NL2, by an hitherto unknown mechanism, is capable of alleviating the inhibition mediated by the SH3 domain. In fact, SH3 domains are widely found protein modules that convey autoinhibitory effects to the proteins bearing them (Pufall \& Graves, 2002). It was therefore very plausible to infer that a direct interaction between NL2 and Collybistin might be responsible for the NL2 mediated activation of $\mathrm{CB} 2 \mathrm{SH} 3+$. This is consistent with my $\mathrm{Y} 2 \mathrm{H}$ data indicating that NL2 is an interaction partner of Collybistin, a feature not shared by NL1 or NL3, despite the fact that all three NLs are capable of binding to Gephyrin (Figure 1).

SH3 domains bind to proline rich ligands (Mayer \& Saksela et al., 2005), and the cytoplasmic tail of NL2 contains several prolines. It is conceivable that NL2 binds to the $\mathrm{SH} 3$ domain directly and subsequently alleviates the inhibition. Indeed, I show in the $\mathrm{Y} 2 \mathrm{H}$ assays that an intact $\mathrm{SH} 3$ domain is necessary and sufficient to mediate binding of Collybistin to NL2 (Figure 1). Interestingly, an alanine substitution of a glycine residue in the $\mathrm{SH} 3$ domain $\left(\mathrm{G} 62 \mathrm{~A}\right.$ in $\left.\mathrm{CB}_{\mathrm{SH}_{3}+}\right)$ leads to loss of binding to NL2. This point mutation was first identified in the human orthologue of Collybistin (hPEM-2; analogous to $\mathrm{CB}_{\mathrm{SH}_{3}}$ ) and was reported to be associated with drug-resistant seizures and hyperekplexia (Harvey et al., 2004). In transfected neurons, it was shown that $\mathrm{CB}_{\mathrm{SH}_{3}} \mathrm{G} 55 \mathrm{~A}$ tends to form large somatic and dendritic aggregates and leads to deficits in Gephyrin and $\mathrm{GABA}_{\mathrm{A}}$ receptor clustering (Harvey et al., 2004). A close look at 
the structure of the $\mathrm{SH} 3$ domain (PDB Code: $2 \mathrm{YSQ}$ ) reveals that this glycine residue is partially buried and lies in close proximity to putative proline-rich ligand-binding sites. Since the inhibitory effect of the $\mathrm{SH} 3$ domain does not change upon G55A substitution (§ 3.1.8), I propose that this mutation causes a global effect on the folding of the $\mathrm{SH} 3$ domain, contrary to what has been suggested previously (Harvey et al., 2004). Yet, it might cause a local misfolding of the SH3 domain and cause deficits in ligand binding, including interactions with NL2 and NL4 (Figure 3).

All NLs have ICDs that are rich in proline; yet it is striking to observe that among the first three paralogues, only NL2 can bind to and activate Collybistin. A close look at the sequence alignment of the ICDs of Neuroligins reveals that NL2 has multiple PXXP motifs and two polyproline stretches of 5 to 8 adjacent proline residues, which are absent in NL1 and NL3. These two proline streches are located downstream of the Gephyrin binding region. A truncation mutant of NL2, bearing a stop codon just upstream of the first polyproline stretch loses binding to Collybistin, while retaining Gephyrin binding (Figure 3). Although the proline residues responsible for direct binding to the $\mathrm{SH} 3$ domain of Collybistin are not yet well defined, I propose that the presence of these proline stretches is a feature of NL2, which distinguishes it from NL1 and NL3 and defines it as a Neuroligin paralogue that is specifically functional at inhibitory synapses.

Interestingly, the NL4 ICD also has a PXXP motif and a stretch of 4 adjacent proline residues, which are upstream of the Gephyrin binding region and are not conserved in other Neuroligin paralogues. This makes NL4 another candidate molecule that can bind to and activate Collybistin. In fact, my colleagues and I found that NL4 is yet another Neuroligin paralogue with a critical role in the assembly of inhibitory synapses (Figure $2 ; \S 4.1 .7$ ). 


\subsubsection{SH3 domain-mediated autoinhibiton of Collybistin}

Although the inhibitory role of the $\mathrm{SH} 3$ domain of Collybistin has been well documented (Kins et al., 2000; Harvey et al.; 2004), the mechanisms underlying this inhibition have been a mystery. One potential mechanism could involve an accessory protein, which binds to the $\mathrm{SH} 3$ domain and blocks the enzymatic functions of Collybistin as a GEF or its $\mathrm{PH}$ domain-mediated membrane recruitment. However, to date, there has been no protein identified as an interaction partner of Collybistin other than Gephyrin and Cdc42. Another mechanism could involve an intramolecular interaction between the $\mathrm{SH} 3$ domain and the downstream $\mathrm{DH}$ and $\mathrm{PH}$ domains, which could cause steric hindrance to interactions mediated by the latter. Such an intramolecular interaction could be responsible for hiding the binding surface from small GTPases and thereby leading to inhibition of the GEF activity; alternatively, it could limit the accessibility of the $\mathrm{PH}$ domain for binding to membrane phosphoinositides, an essential feature of Collybistin for recruiting Gephyrin to the plasma membrane. In fact, many of the Dbl-family proteins were shown to possess a regulatory domain or short stretch of amino acids that inhibit GEF activity via an intramolecular interaction. This was established in a variety of studies on Dbl-family GEFs, where deletion of these inhibitory domains or sequences, which were found mostly located N-terminally (but in a few cases also C-terminally) of the $\mathrm{DH} / \mathrm{PH}$ tandem domains, results in constitutive activation of the GEF. Dbl-family proteins that are regulated by autoinhibition include Vav (Aghazadeh et al., 2000; Yu et al., 2010), Tim (Yohe et al., 2007), ASEF (Kawasaki, et al., 2000), Dbl (Ron et al., 1989), Tiam1 (van Leeuwen, 1995), Ect2 (Miki et al., 1993), Net1 (Chan et al., 1996) (autoinhibition mediated by a N-terminal element), p115RhoGEF (Wells et al., 2001), p63RhoGEF (Rojas et al., 2007) and Lbc (Sterpetti et al., 1999) (autoinhibition mediated by a C-terminal element). Therefore the use of intramolecular inhibitory interactions seems to be a common mechanism in GEF regulation. 
A wide range of proteins, including Tec-family tyrosine kinases (Andreotti et al., 1997; Pursglove et al., 2002), Src-family tyrosine kinases (Moarefi et al., 1997; Lerner et al., 2002), nuclear protein tyrosine kinase c-Abl (Barila et al., 1998, Pluk et al., 2002), the synaptic scaffolding molecules PSD-95 (Tavares et al., 2001) and SAP102 (Seabold et al., 2003) and a NADPH oxidase p47 ${ }^{\text {phox }}$ (Ago et al., 2003; Yuzawa et al., 2004), are regulated by intramolecular SH3 domain/PXXP interactions, indicating that the $\mathrm{SH} 3$ domain is a widely used autoinhibitory module. Interestingly, among the whole family of Dbl-family proteins, 22 of them, including Collybistin, contain an SH3 domain, yet only eight out of these contain a polyproline motif. Among those, Kalirin (Schiller et al., 2006), Tim and NGEF (Yohe et al., 2008) are inhibited by intramolecular SH3/PXXP interactions. Some other SH3 domain containing Dbl-family proteins, including Asef and Intersectin, are inhibited by the $\mathrm{SH} 3$ domain through direct binding to the $\mathrm{DH} / \mathrm{PH}$ tandem domain interface, independently of proline motifmediated interactions (Zamanian et al., 2003; Mitin et al., 2007; Murayama et al., 2007). Altogether, this implies that SH3 domains can regulate Dbl-family proteins in a polyproline-dependent or -independent manner. The isoform of Collybistin that was studied here, $\mathrm{CB} 2_{\mathrm{SH}_{3+1}}$ does not contain any proline motif, therefore it is likely that Collybistin is regulated by a non-polyproline mediated autoinhibition. However, another splice isoform of Collybistin, $\mathrm{CB}_{\mathrm{SH}_{3+}}$, which is equally abundant in vivo and is equivalent to the human orthologue of Collybistin (hPEM-2) (Harvey et al., 2004), contains a proline rich motif (PPSYPPP) at its C-terminal coiled coil region, which is alternatively spliced in $\mathrm{CB} 2_{\mathrm{SH}_{3}+}$. Whether this proline stretch is involved in an intramolecular interaction with the $\mathrm{SH} 3$ domain and can thus account for a splice isoform specific mode of autoinhibition remains to be identified.

Asef and Collybistin are not only close homologues sharing over $80 \%$ sequence homology; they also share an almost identical domain organization. A close look at the crystal structure of Asef reveals the presence of an extensive interface formed between the $\mathrm{SH} 3$ domain and the $\mathrm{DH} / \mathrm{PH}$ tandem domains (Mitin et al., 2007; Murayama et al., 2007). In this autoinhibited conformation, Cdc42 
binding and activation is hindered. Interestingly, binding of the armadillo repeats of the tumor suppressor protein Adenomatous Polyposis Coli (APC) to an APC binding motif located $\mathrm{N}$-terminally to the $\mathrm{SH} 3$ domain, or truncation of the $\mathrm{SH} 3$ domain of Asef, relieves autoinhibition and leads to specific activation of Cdc42 (Mitin et al., 2007). It was suggested that upon binding to APC, Asef undergoes a conformational switch towards a more open state in which the $\mathrm{SH} 3$ domain can no longer mask the Cdc42-binding surface of the $\mathrm{DH}$ domain and thereby is capable of performing its enzymatic activity.

In the present study, I investigated whether the ability of Collybistin in targeting Gephyrin to the plasma membrane is autoinhibited by the $\mathrm{SH} 3$ domain in a similar way, and whether NL2-mediated activation of Collybistin happens in an analogous mode to APC-mediated Asef activation. Using a cell-based mutagenesis assay, I found that Collybistin indeed shares similarities with Asef in the mechanism of autoinhibition and activation. To examine both mechanisms, two different sets of mutations were incorporated into Collybistin.

The first set of mutations, which were selected on the basis of Asef structure, included alanine substitutions of some of the residues from the $\mathrm{SH} 3$ and $\mathrm{DH}$ domains of Collybistin, which are crucial for the formation of the SH3-DH/PH interface in Asef. These point mutations were expected to abolish the potential intramolecular interaction in Collybistin and result in loss of the inhibiting impact of the $\mathrm{SH} 3$ domain on Gephyrin recruitment to the plasma membrane. My data clearly indicate that one of these point mutations, E262A, renders Collybistin constitutively active (Figure 5). According to the Asef structure (Mitin et al., 2007) the glutamic acid residue that belongs to the fifth helix of the $\mathrm{DH}$ domain ( $\alpha 5)$, is capable of forming an electrostatic bridge with R249 (R70 in $\mathrm{CB} 2_{\mathrm{SH}_{3}+}$ ) and $\mathrm{E} 291$ (E117 in $\left.\mathrm{CB} 2_{\mathrm{SH}_{3}+}\right)$. It is also capable of forming hydrogen bonds to the backbone atoms of V252 (V73 in $\mathrm{CB}_{\mathrm{SH}_{3}}$ ) and N253 (N74 in $\left.\mathrm{CB} 2_{\mathrm{SH}_{3}+}\right)$. This network of polar interactions seems to be responsible for sealing the $\mathrm{SH} 3$ to the $\mathrm{DH} / \mathrm{PH}$ interface. Therefore, alanine substitution of E262 might 
have drastic consequences, leading to unfastening of the remainder of the interface and constitutive activation of Collybistin.

The second set of point mutations were generated on the residues that form the core PXXP binding surface of the SH3 domain, in order to test whether it is possible to render Collybistin irresponsive to NL2 mediated activation by interfering with the putative NL2 binding residues. Alignment of $\mathrm{SH} 3$ domains from a vast variety of proteins reveals the presence of an essential conserved tryptophan residue ( $\mathrm{W} 52$ in $\mathrm{CB}_{\mathrm{SH}_{3}}$ ). This tryptophan residue has been shown to mediate proline-rich ligand binding (Macias et al., 2002). An aromatic residue that also contributes to PXXP binding usually occupies the $\beta 4$ loop of the $\mathrm{SH} 3$ domain. This residue is another tryptophan in $\mathrm{CB}_{\mathrm{SH}_{3+}}$ (W63). Interestingly, both of these tryptophan residues lie in close vicinity of G62, the residue responsible for triggering hyperekplexia in human when substituted to alanine, and which we show also to negatively affect NL2 binding. In fact, comparison of $\mathrm{CB}_{\mathrm{SH}_{3}-}, \mathrm{CB}_{\mathrm{SH}_{3}+}$ and its point mutants in pulldowns indicated that similar to G62A, W52A and W63A are also deficient in binding to NL2, although they all have preserved Gephyrin binding. The cell-based mutagenesis assay revealed that all three point mutants appear to have deficits in being activated by NL2 and tethering Gephyrin to the plasma membrane (Figure 6). Altogether, I show that interfering with the NL2/SH3 domain interaction causes Collybistin to stay tightly associated with Gephyrin aggregates in the cytoplasm, indicating that NL2 binding is indispensable for Collybistin activation.

In order to validate the dependence of Collybistin activation on the NL2Collybistin interaction, the NL2 binding deficient mutants of Collybistin were transfected in dissociated hippocampal cultures from Collybistin $\mathrm{KO}$ mice (Figure 10). Among the three mutants tested in this context, G62A caused the most severe impairments in the rescue of Gephyrin clustering. This mutation caused the formation of large somatic and dendritic aggregates of Collybistin where Gephyrin was also sequestered, not allowing for redistribution of Gephyrin to postsynaptic puncta. Since the effect of this mutation was not 
confined to somatic areas where NL2 is required for Gephyrin clustering, but rather spread over to dendritic areas as well, it can be stated that alanine substitution of the G62 leads to more severe defects in the $\mathrm{SH} 3$ domain structure, which does not only prevent NL2 binding. In fact, in a recent study it was shown that $\mathrm{GABA}_{A} \mathrm{R} \alpha 2$ subunit is also capable of binding to the $\mathrm{SH} 3$ domain in a non-canonical manner, independent of PXXP-SH3 binding, which is also impaired by the G62A mutation (Saiepour et al., 2010), indicating that this mutation may interfere with other, yet unknown mechanisms that involve Collybistin. Unexpectedly, and in contrast to heterologous cell-based assays, upon expression in Collybistin KO neurons, except for G62A, the other NL2 binding-deficient point mutants of Collybistin were sufficient to rescue Gephyrin clustering both in somatic and in dendritic compartments. This unexpected effect might be due to the strong overexpression of the exogeneous Collybistin constructs, which may become constitutively active upon expression in very high densities. In fact, it was anecdotally observed in COS7 cells that expression of exogeneous Collybistin constructs for longer than 16h leads to autoactivation of $\mathrm{CB} 2_{\mathrm{SH}_{3}+}$ and emergence of submembrane Gephyrin microaggregates even in the absence of NL2. Therefore, to validate the effect of the W52A and W63A mutations in a neuronal context, a more controlled and moderate expression of the transfected constructs might be necessary, e.g. using lentiviral constructs.

\subsubsection{PH domain-mediated membrane tethering of Collybistin}

Dbl-family proteins consistently contain a $\mathrm{PH}$ domain in tandem with their $\mathrm{DH}$ domain. $\mathrm{PH}$ domains are protein modules of around 120 residues and are present in a vast variety of proteins with roles in cell signaling and cytoskeletal rearrangements (Haslam et al., 1993). DH domains are sufficient to catalyze nucleotide exchange, but inclusion of the adjacent $\mathrm{PH}$ domain enhances the exchange activity (Rossman et al., 2000). Although PH domains share low sequence homology, they all contain a $\beta$-sandwich fold supported by a $\mathrm{C}$ terminal helix. Studies on many other proteins demonstrated that $\mathrm{PH}$ domains interact with membrane phosphoinositides with a wide degree of affinities and 
specificities via basic residues within loops located between the $\beta 1 / \beta 2$ and $\beta 3 / \beta 4$ strands (Lemmon et al., 1995). The PH domains of many of the Dblfamily proteins have varying affinities for different phosphoinositide derivatives. However, except for Asef and Dbs (Muroya et al., 2007; Kawasaki et al., 2009; Baumeister et al., 2006) no other member of this family was shown to be localized to distinct cellular compartments via phosphoinositide binding.

The significant role of the $\mathrm{PH}$ domain of Collybistin in membrane recruitment and synaptic targeting of Gephyrin has been demonstrated, as the deletion of this domain from the constitutively active Collybistin variant, $\mathrm{CB} 2_{\mathrm{SH}_{3}-} \Delta \mathrm{PH}$, results in accumulation of cytoplasmic Gephyrin-Collybistin coaggregates in heterologous cells, and loss of synaptic Gephyrin clusters upon expressing this truncation construct in rat cortical neurons (Harvey et al., 2004). Furthermore, a patient presenting epileptic seizures, increased anxiety and mental retardation was identified to carry a balanced chromosomal translocation, which leads to the disruption of the Collybistin gene and expression of a truncated version of Collybistin lacking the PH domain (Kalscheuer et al., 2009). In the current study, the critical role of the $\mathrm{PH}$ domain in membrane recruitment of Gephyrin/Collybistin complexes was validated (Figure 7). A construct where the entire $\mathrm{PH}$ domain was deleted in the $\mathrm{CB} 2_{\mathrm{SH}_{3+}}$ backbone is deficient in targeting Gephyrin to the plasma membrane and is irresponsive to activation by NL2, indicating that an intact $\mathrm{PH}$ domain is essential for the formation of submembraneous Gephyrin microaggregates and NL2 itself is not capable of tethering Gephyrin/Collybistin complex to the plasma membrane in the absence of the $\mathrm{PH}$ domain. However, since the $\mathrm{PH}$ domain is a functional module of Collybistin, which may bind to other adaptor proteins as well as to membrane phosphoinositides, the $\mathrm{PH}$ domain was screened for point mutations to abolish membrane tethering. The cell-based assays demonstrated that the positively charged residues from the $\beta 3 / \beta 4$ loop of the $\mathrm{PH}$ domain are essential for membrane targeting of Collybistin. 
Previous studies showed that Collybistin specifically interacts with phosphatidylinositol-3-phosphate (PI3P) (Kalscheuer et al., 2009; Reddy-Alla et al., 2010), which has been validated in the current study. Strikingly, compared to full length $\mathrm{CB} 2_{\mathrm{SH}_{3+1}}$ the $\Delta \mathrm{SH} 3$ mutant of Collybistin shows significantly stronger binding to PI3P, as indicated by protein-lipid overlay assays (Figure 8). This observation is the first indication that the $\mathrm{SH} 3$ domain-mediated autoinhibition in Collybistin may lead to the reduced affinity of the $\mathrm{PH}$ domain to membrane phosphoinositides due to steric interference imposed by the $\mathrm{SH} 3$ domain. In fact, in the autoinhibited Asef structure, the $\beta 3 / \beta 4$ loop of the $\mathrm{PH}$ domain is not too far away from the occluded surface of the $\mathrm{SH} 3-\mathrm{DH} / \mathrm{PH}$ interface, while one of the lysine residues that is essential for targeting Collybistin to the plasma membrane (K533 in Asef, $\mathrm{K} 358$ in $\mathrm{CB}_{\mathrm{SH}_{3} ;}$ § 3.1.6) is actually involved in an intramolecular interaction, where it forms a salt bridge with an aspartic acid residue of the SH3 domain (Mitin et al., 2007). Although this aspartic acid is replaced by an asparagine in Collybistin (N31 in $\mathrm{CB}_{\mathrm{SH}_{+}}$), it is conceivable that the $\mathrm{SH} 3-\mathrm{DH} / \mathrm{PH}$ interface may interfere with the electrostatic interactions of the positively charged $\beta 3 / \beta 4$ loop residues of the $\mathrm{PH}$ domain with membrane phosphoinositides, leading to inhibition of the membrane tethering of Collybistin and restricting it to the cytoplasm. A point mutant of Collybistin, $\mathrm{CB} 2_{\mathrm{SH}_{3}+} \mathrm{E} 262 \mathrm{~A}$, which was identified to be constitutively active in cell-based assays, showed increased binding to PI3P in the proteinlipid overlay assays compared to $\mathrm{CB}_{\mathrm{SH}_{3+}}$. This observation provides an additional line of evidence that $\mathrm{SH} 3$ domain-mediated autoinhibition is directed towards the $\mathrm{PH}$ domain binding to membrane phoshphoinositides.

In the present study, I have shown that NL2 binding is necessary for alleviating $\mathrm{SH} 3$ domain-mediated autoinhibition in Collybistin. Analogous to Asef activation by APC (Mitin et al., 2007), binding of the proline-rich ICD of NL2 might induce a conformational change in Collybistin that results in detachment of the $\mathrm{SH} 3$ and $\mathrm{DH} / \mathrm{PH}$ domain interface and thus increase membrane accessibility of the $\mathrm{PH}$ domain. The positive effect of NL2 binding on the PI3P binding of Collybistin is demonstrated by the PI3P-NL2 cospotting assays (Figure 
9). The dual presence of PI3P and recombinant NL2cyt in a restricted environment leads to enhanced binding of $\mathrm{CB} 2_{\mathrm{SH} 3+}$ to the PI3P/NL2 spots, in contrast to individual PI3P or NL2 containing spots. The $\triangle \mathrm{PH}$ mutant of $\mathrm{CB} 2_{\mathrm{SH} 3+}$ did not respond to NL2 activation in this assay, indicating that an intact $\mathrm{PH}$ domain is essential for Collybistin to be attached to PI3P/NL2 spots, and binding to NL2 alone is not sufficient for improved interaction with these spots. On the other hand, attachment of the $\Delta \mathrm{SH} 3$ mutant to PI3P/NL2 spots was not significantly increased compared to the PI3P spots, indicating that the presence of NL2 stimulates PI3P binding of Collybistin only in the presence of an intact $\mathrm{SH} 3$ domain. Interestingly, transfection of the GFP-fused ICD of NL2 in mammalian cells did not lead to activation of Collybistin and membrane tethering of Gephyrin (data not shown). Similar to this observation, preincubating the recombinant NL2cyt with $\mathrm{CB} 2_{\mathrm{SH} 3+}$ was also not effective in stimulating phosphoinositide binding (not shown). It thus appears that in order to mediate its allosteric effect, NL2 needs to be attached to a fixed surface, in close proximity to membrane phosphoinositides. In the cell based assay, NL2 is presented on the cell surface as a transmembrane protein, where it can locally activate Collybistin and initiate PI3P binding. In the PI3P/NL2 cospotting assay, along with PI3P molecules, recombinant NL2 cytoplasmic tail is fixed on a nitrocellulose membrane surface, where it can induce an allosteric effect on Collybistin that would lead to the attachment of Collybistin to the PI3P molecules that are adjacent to NL2 molecules.

Upon further investigation of this in vitro PI3P binding assay, I could show that NL2 mediated activation of Collybistin is dependent on NL2 concentration, leading to a sigmoidal curve, which is characteristic for cooperative binding. It can thus be postulated that NL2 binding to the $\mathrm{SH} 3$ domain leads to an allosteric effect on Collybistin, which enhances the affinity of the $\mathrm{PH}$ domain for PI3P. This steep curve implies that the inactive and active states of Collybistin may represent a binary condition between "on" and "off" states. By binding to the SH3 domain, NL2 can switch Collybistin to a PI3P bound "on" state, which leads to nucleation of the Gephyrin scaffold in vivo. In this PI3P/NL2 cospotting 
assay, the kinetics of Collybistin activation implies that the density of NL2 on the 2D surface might determine whether this mechanism is on or off. This dose dependence may have parallels with the synaptic clustering of NL2. It has been shown that in neurons, surface clustering of exogeneous NL2 by antibodycoated beads induces Gephyrin recruitment (Graf et al., 2004). Additionally, it has recently been shown that two forms of Neurexin, $\alpha$ and $\beta$, act synergistically during the formation of perisomatic inhibitory synapses in cortical neurons, where $\alpha$-neurexins establish initial contacts with postsynaptic adhesion molecules, and $\beta$-neurexins strengthen these connections by binding to and clustering NL2 (Fu et al., 2010). These studies indicated that the validation and maturation of synaptic contacts require Neurexin-dependent accumulation and dense clustering of Neuroligins at the postsynaptic membrane. Reminiscent of the local synaptic accumulation of NL2 via transsynaptic interactions in vivo, in the PI3P/NL2 cospotting assay the local density of NL2 is observed to be an important factor for switching Collybistin to an PI3P-bound "on" state.

Phosphoinositides function as second messengers, which have roles in cell signaling, vesicle trafficking and cytoskeletal dynamics. PI3P, a product of PI3Kinase, is a phosphoinositide derivative that is responsible for regulating endosomal trafficking. The constitutive pool of PI3P, which is produced by class III phosphotidylinositol-3-kinase (PI3K), is specifically localized to endosomal vesicles (Gillooly et al., 2000). In addition, more dynamic pools of PI3P molecules can be generated by cellular stimulation in other cellular compartments, and these PI3P molecules are involved in other signaling pathways (reviewed in Falasca \& Maffucci, 2006 and Falasca \& Maffucci, 2009). It has been demonstrated that dynamic pools of PI3P are generated in the plasma membrane upon activation and membrane recruitment of PI3K-C2 $\alpha$ in response to insulin stimulation (Maffucci et al., 2003; Falasca et al., 2007). This kinase belongs to the class II PI3Ks, different from class III PI3Ks that are responsible for generating the constitutive pool of $\mathrm{PI3P}$ in endosomal compartments. Following this rationale, it is plausible that a specific signaling event at inhibitory synapses might be responsible for activating a specifc, yet 
unknown, class II PI3K that leads to local generation of PI3P confined to the inhibitory postsynaptic membrane where the Gephyrin scaffold can be tethered via the NL2-activated Collybistin. It remains to be tested whether this potential mechanism of dynamic PI3P production at the synaptic membrane involves signaling events initiated by NL2 or Collybistin and whether it is regulated by synaptic activity.

\subsubsection{The putative catalytic role of the DH domain}

Collybistin stands out as an adaptor molecule for establishing a physical link between the plasma membrane and the Gephyrin scaffold. In the present study, it is proposed that the NL2-induced conformational switch in Collybistin allows for binding to phosphoinositides and membrane tethering via the $\mathrm{PH}$ domain, which is otherwise occluded by the SH3 domain. However, the possibility that NL2 binding also increases the GEF activity of Collybistin cannot be excluded. Since it was shown for other Dbl-family proteins that the $\mathrm{SH} 3$ domain is capable of blocking the GTPase binding to the DH domain (Mitin et al., 2007) and due to the fact that $\mathrm{CB}_{\mathrm{SH}_{3-1}}$ compared to $\mathrm{CB}_{\mathrm{SH}_{3+} \text {, }}$ is a more potent activator of Cdc42 (Xiang et al., 2006), an NL2 induced conformational switch in Collybistin may also allow for better binding of GTPases to the $\mathrm{DH}$ domain. According to a previously proposed model, activated Collybistin may initiate signaling events via its GEF activity, which may regulate the deposition of the Gephyrin scaffold at inhibitory synapses (Kneussel \& Betz, 2000).

Cdc42 is thought to be the only small G-protein that Collybistin can selectively bind to and activate (Reid et al., 1999; Xiang et al., 2006). To examine whether Cdc42 signaling is involved in Gephyrin clustering, a wide variety of approaches have been carried out. An alanine substitution of the Glu117 of $\mathrm{CB} 2_{\mathrm{SH}_{3+}}$ equivalent to Glu57 of $\mathrm{CB} 2_{\mathrm{SH}_{3} \text {, }}$ a residue that establishes hydrogen bonds with three independent residues of Cdc42 (Xiang et al., 2006), did not lead to perturbations in membrane targeting of Gephyrin upon activation of Collybistin via NL2 (data not shown). Parallel to this observation, expression of 
the dominant negative mutant of $\mathrm{Cdc} 42(\mathrm{~T} 17 \mathrm{~N})$ along with $\mathrm{CB} 2_{\mathrm{SH} 3+}$ Gephyrin and NL2 did not lead to any observable changes in the membrane coclustering of NL2 and Gephyrin at the plasma membrane, while the constitutively active Cdc42 mutant $(\mathrm{Q} 61 \mathrm{~L})$ was deficient in replacing Collybistin for mediating the membrane recruitment of Gephyrin and its association with NL2 (Poulopoulos, unpublished observation). More recently, it was shown that a $\mathrm{CB} 2_{\mathrm{SH}_{3}-}$ mutant that lacks GEF activity towards Cdc42 was still functional in the membrane recruitment of Gephyrin in heterologous and cultured neuronal cells. Moreover, deletion of the Cdc42 gene in the mouse forebrain did not lead to deficits in the assembly of the Gephyrin scaffold and $\mathrm{GABA}_{A} \mathrm{R}$ clustering (Reddy-Alla, et al., 2010). These observations indicate that membrane tethering of the Gephyrin scaffold via NL2-activated Collybistin does not involve the putative GEF activity of Collybistin on Cdc42.

The Dbl-family of proteins appears to have evolved towards specificity for Rhofamily GTPases (Schmidt and Hall, 2002). However among the over 20 GTPases identified in the eukaryotic genome so far (Bustelo et al., 2007), only Rho, Rac and Cdc42 have been tested for activation by Dbl-family GEFs. Therefore, even though its putative GEF activity for Cdc42 is dispensable for the synaptic function of Collybistin, there may be other candidate GTPases that Collybistin could act upon, leading to signaling events that are important for the proper assembly of the inhibitory postsynaptic apparatus. It remains to be tested whether Collybistin is capable of facilitating nucleotide exchange on other Rhofamily GTPases, which may lead to initiation of signaling cascades necessary for inhibitory postsynapse organization.

\subsubsection{Completing the puzzle: The tripartite complex of NL2- Gephyrin-Collybistin}

The tripartite complex of NL2, Gephyrin and Collybistin constitutes the basic unit of the protein-protein interaction network required to initiate the assembly 
of inhibitory postsynapses (Poulopoulos et al., 2009). All the members of this tripartite complex appear to interact with each other. In order to elucidate the mechanism of the assembly of the tripartite complex of NL2, Gephyrin and Collybistin and to further understand the NL2 induced activation of Collybistin, followed by the tethering of the Gephyrin scaffold to the plasma membrane, it is of great interest to identify the interaction sites on each protein.

4.1.5.1. NL2 - Gephyrin interaction: NL2 interacts with the E-domain of Gephyrin. This domain is responsible for mediating interactions of Gephyrin with various other postsynaptic proteins including Collybistin. It is also essential for the homodimerization of the monomeric Gephyrin units. The exact binding site for NL2 on Gephyrin E-domain remains to be identified. On the NL2 side, a 15-amino acid long Gephyrin binding motif was identified, which is necessary and sufficient for interaction with Gephyrin (Poulopoulos et al., 2009).

4.1.5.2. NL2 - Collybistin interaction: I showed here that NL2 and NL4 can specifically interact with the SH3 domain of Collybistin, an ability that is not shared by NL1 or NL3. These data provide evidence for the specific roles of NL2 and NL4 at the inhibitory synapse. Due to the proline-rich nature of the cytoplasmic tails of NL2 and NL4, I postulate that the interaction of Collybistin with NL2 and NL4 might occur in a conventional SH3-PXXP interaction mode. Indeed, replacement of surface exposed hydrophobic residues in the $\mathrm{SH} 3$ domain (W52 and W63) by alanines resulted in the loss of interaction with NL2, showing that these residues are partly responsible for binding to NL2. Additionally, truncation mutation of the proline rich C-terminal tail of NL2 downstream of the P798 leads to the loss of interaction with the SH3 domain. The $\mathrm{SH} 3$ binding region on the NL4 cytoplasmic tail remains to be identified.

\subsubsection{Gephyrin-Collybistin interaction: Gephyrin interacts with Collybistin} via a stretch of residues that span the border between the linker region and the E-domain (Harvey et al., 2004). The significance of these residues for binding to Gephyrin was validated in the present study, and it was shown that the triple 
alanine substitution of the residues P320, F321 and P322 leads to lack of Collybistin-Gephyrin coclustering in COS7 cells and cause deficits in membrane targeting of Gephyrin (data not shown). On the Collybistin side, it has been proposed that a group of charged residues (R107, D108, R111 and $\mathrm{E} 117$ ) at the $\mathrm{N}$-terminal part of the $\mathrm{DH}$ domain are responsible for mediating the interaction with Gephyrin. A quadruple alanine substitution of these residues was shown to affect coclustering of Gephyrin and Collybistin in transfected HEK 293 cells (Grosskreutz et al., 2001). However, this finding was later contested; a triple R107A-D108A-E117A mutant of Collybistin retains its ability to bind to Gephyrin and a single R111A mutation leads to descreased solubility, probably due to structural perturbations (Xiang et al., 2006). Therefore, it was suggested that the quadruple alanine substitutions in Collybistin actually lead to local structural rearrangements, rather than to specific loss of binding to Gephyrin. In order to clearly identify the Gephyrin binding surface, a more detailed biochemical analysis needs to be performed. I have taken here significant steps toward the dissection of the Gephyrin-Collybistin interaction.

The Collybistin residues that were proposed by Grosskreutz et al., (2001) to be responsible for Gephyrin binding raised the possibility that Asef, the closest homologue of Collybistin, may also share the Gephyrin binding characteristics of Collybistin, since these two proteins share above $60 \%$ identity in their primary sequences. Interestingly, three out of these four candidate residues (D108, R111, E117) for Gephyrin binding are conserved in Asef, while R107 is conservatively replaced by a lysine. Despite the high sequence homology and structural similarity, a recombinant Asef construct, covering the $\mathrm{DH}$ and $\mathrm{PH}$ domains (analogous to $\mathrm{CB} 2_{\mathrm{SH}_{3}}$ ) was not able to bind to Gephyrin in vitro (Figure 11). Additionally, neither full-length Asef, nor its domain deletion constructs (Asef $\triangle \mathrm{ABR}$, equivalent to $\mathrm{CB} 2_{\mathrm{SH} 3+}$ and Asef $\Delta \mathrm{ABR}-\Delta \mathrm{SH} 3$, equivalent to $\mathrm{CB} 2_{\mathrm{SH}_{3}}$ ) were able to cocluster with Gephyrin in transfected COS7 cells. This finding indicates that even if the proposed residues (R107, D108, R111, E117) contribute to the Collybistin-Gephyrin interaction, they do not constitute a sufficient Gephyrin binding motif. 
Even though it does not appear to function as an alternative GEF for membrane clustering of Gephyrin, Asef, with its structural similarity to Collybistin, provided essential hints about the mechanisms of Collybistin function (see above). Based on the observation that Asef can neither bind to nor induce membrane targeting of Gephyrin, I speculate that the minor differences in the primary structures of Asef and Collybistin may underlie the specific postsynaptic functions of Collybistin. In order to study the Gephyrin-Collybistin interaction in more detail and to identify the domains and motifs on Collybistin responsible for mediating Gephyrin binding, sequence patches of Collybistin were replaced by the homologous regions of Asef. The interaction data with the chimeric CollybistinAsef constructs indicate that the Gephyrin binding sequence on Collybistin lies between the residues $\mathrm{A} 30$ and K130. Interestingly, within this 100-residue segment, the major differences between Asef and Collybistin are located in the linker region between the $\mathrm{SH} 3$ and the $\mathrm{DH}$ domains. Since $\mathrm{CB} 2_{\mathrm{SH}_{3}-}$ is also capable of binding to Gephyrin, it can be inferred that at least one binding site for Gephyrin lies between the residues $\mathrm{L} 71$ and $\mathrm{K} 130$. This putative binding region is most likely responsible for binding to the E-domain of Gephyrin on residues P320, F321 and $\mathrm{P} 322$, since neither $\mathrm{CB}_{\mathrm{SH}_{3}-}$ nor $\mathrm{CB} 2_{\mathrm{SH} 3+}$ were able to bind to a P320A-F321A-P322A mutant of Gephyrin.

Although both $\mathrm{CB} 2_{\mathrm{SH}_{3}-}$ and $\mathrm{CB} 2_{\mathrm{SH}_{3}+}$ have the capacity to bind to Gephyrin, the possibility of an additional Gephyrin binding site on the $\mathrm{SH} 3$ domain cannot be excluded. In fact, the $\mathrm{SH} 3$ domain alone was shown to be sufficient to bind to Gephyrin (Figure 11F). It is not yet precisely known whether the SH3 domain has the capacity to bind to parts of Gephyrin other than the E-domain. It is also unknown whether this interaction follows a canonical SH3 to PXXP mode of binding, which is plausible due to the presence of multiple polyproline stretches on the G- and linker domains of Gephyrin. Regardless of its nature, it is conceivable that this interaction is not responsible for releasing the $\mathrm{SH} 3$ domain-mediated autoinhibition of Collybistin, since the Gephyrin-Collybistin complex is retained in the cytoplasm in transfected mammalian cells. On the contrary, the Gephyrin-SH3 domain interaction might be responsible for 
potentiating the inhibiting effect of the SH3 domain, and Gephyrin binding on the SH3 domain may partially attenuate NL2 binding. Such a mechanism could guarantee that Collybistin is retained in a closed and inactive conformation and can be activated only when NL2 is present to compete out Gephyrin for binding to the SH3 domain. The significance of the Gephyrin-SH3 domain interaction remains to be clarified.

\subsubsection{A mechanistic model underlying the assembly of perisomatic inhibitory synapses}

Based on the recent findings on the role of NL2 in inhibitory postsynaptic assembly (Poulopoulos et al., 2009), together with the protein-protein, proteinlipid interactions and the cell based mutagenesis assays presented in this study, a molecular model for the assembly of the Gephyrin scaffold and the subsequent clustering of inhibitory neurotransmitter receptors at perisomatic inhibitory postsynapses can be proposed (Schema 8). According to this model, the trimeric Gephyrin units are transported to synaptic sites together with Collybistin. During this transport process Collybistin adopts a closed conformation that hinders the phosphoinositide binding capacity of its $\mathrm{PH}$ domain. At postsynaptic sites, NL2 molecules, clustered by Neurexins at the surface of the plasma membrane, initially bind to cytoplasmic GephyrinCollybistin complexes via the Gephyrin binding motif, bringing the scaffolding complex in transient interaction with NL2. Subsequently, the NL2 ICD binds to the $\mathrm{SH} 3$ domain of Collybistin and the assembly of this tripartite complex between NL2, Gephyrin and Collybistin leads to the activation of Collybistin by a conformational switch mechanism. The NL2-induced removal of the SH3 domain from the arch-like structure formed by the tandem $\mathrm{DH} / \mathrm{PH}$ domains alleviates the autoinhibiting effect of this domain and allows for the $\mathrm{PH}$ domain 


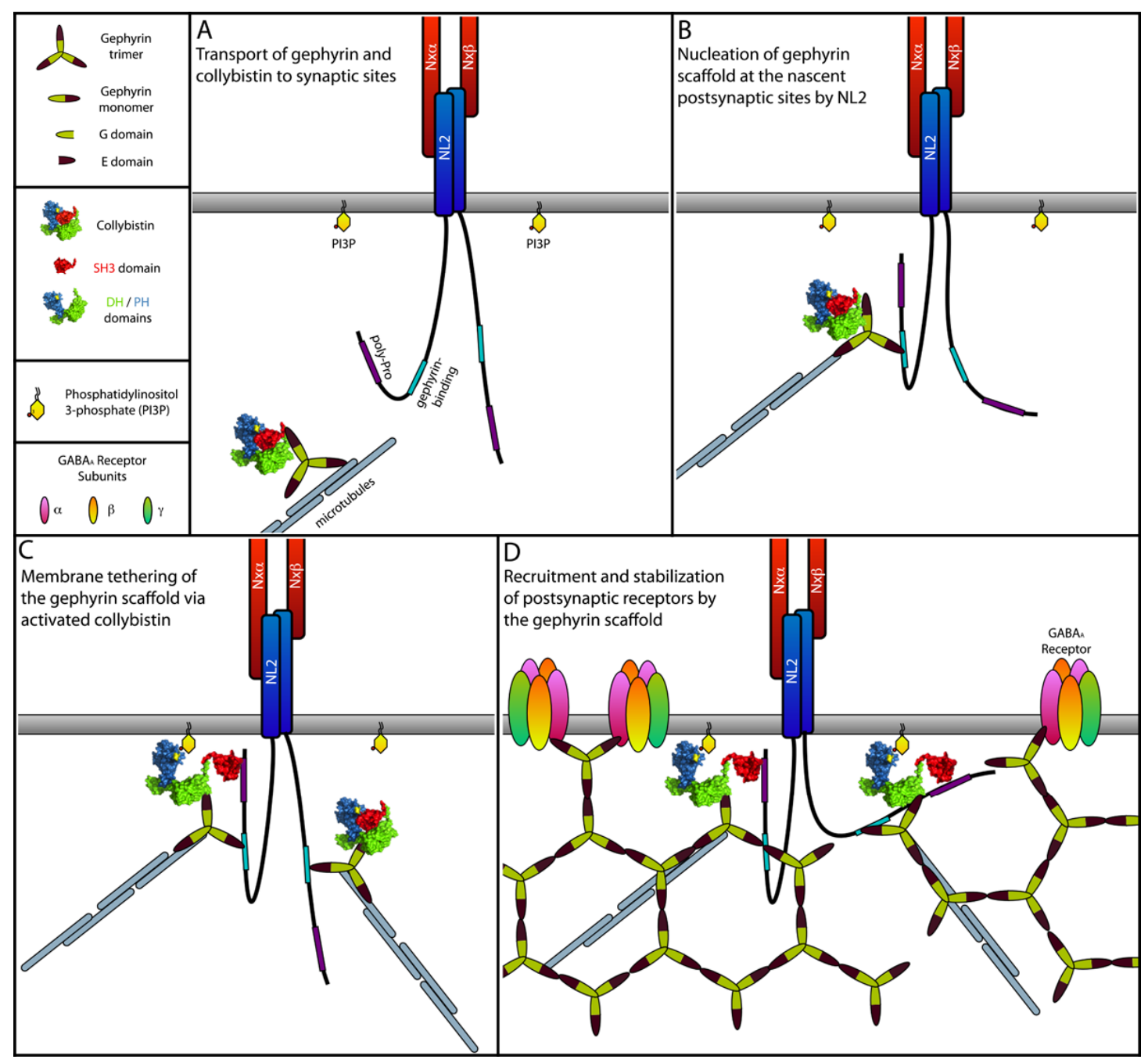

Schema 8: Assembly model for the perisomatic inhibitory synapses. (A) Gephyrin trimers associated with Collybistin are transported to subsynaptic sites along the microtubules. During transport, Collybistin adopts a closed conformation, in which the $\mathrm{SH} 3$ domain folds back onto the tandem DH/PH domains, similar to what is known for the autoinhibited form of Asef, the closest homologue of Collybistin (Mitin et al., 2007; Kawasaki et al., 2007). In this closed inactive conformation, both the catalytic activity of the $\mathrm{DH}$ domain and binding of the $\mathrm{PH}$ domain to membrane phosphoinositides are suppressed due to the hindrance imposed by the SH3 domain. (B) At postsynaptic sites, NL2 molecules, clustered by presynaptically expressed Neurexins, interact transiently with the cytoplasmic Gephyrin-Collybistin complex. This interaction brings the proline-rich cytoplasmic domain of NL2 into close vicinity of the SH3 domain of Collybistin. (C) A typical SH3 domain-ligand interaction mediated by a PXXP motif in the cytoplasmic domain of NL2 leads to a structural rearrangement in Collybistin, resulting in a more open conformation of this GEF. Similar to the APC mediated activation of Asef (Mitin et al., 2007), binding of NL2 is thought to interfere with the intramolecular interactions between the $\mathrm{SH} 3$ and the $\mathrm{DH} / \mathrm{PH}$ domains. This conformational switch towards an open state now allows the $\mathrm{PH}$ domain to associate with plasma membrane-bound PI3P, which might be generated at the inhibitory postsynaptic membrane as a product of a yet unknown signaling mechanism (see text) initiated either by NL2, Collybistin or another inhibitory synaptic molecule. Once attached to PI3P, Collybistin preserves its membrane bound open conformation and tethers Gephyrin to the plasma membrane. (D) $\mathrm{GABA}_{\mathrm{A}} \mathrm{Rs}$ are recruited via potential links to Gephyrin-Cb-NL2 complexes. 
to have an increased binding capacity for membrane associated PI3P, which is probably locally generated as a product of a signaling mechanism initiated by NL2, Collybistin or another inhibitory synaptic molecule. During this molecular switch mechanism, rearrangements in the Collybistin structure may lead to detachment of the $\mathrm{SH} 3$ domain from $\mathrm{DH} / \mathrm{PH}$ domains, enabling the positively charged residues of the $\beta 3 / \beta 4$ loop of the $\mathrm{PH}$ domain to establish electrostatic connections to PI3P molecules. Once attached to PI3P, Collybistin probably retains its membrane bound open conformation and the tethering of the Gephyrin scaffold indirectly via the $\mathrm{PH}$ domain of Collybistin to the plasma membrane allows for additional Gephyrin-Collybistin complexes to be recruited to these sites, which were initially nucleated by the NL2-Gephyrin interaction. Deployment of the Gephyrin scaffold allows for the recruitment and accumulation of the $\gamma 2$ and $\alpha 2$ subunit containing $\mathrm{GABA}_{\mathrm{A}}$ receptors, which are dependent on Gephyrin clustering, in apposition to presynaptic terminals of GABA release.

In the proposed model, the $\mathrm{SH} 3$ domain of Collybistin functions as trigger for a switch mechanism, which is turned on specifically by the ICDs of NL2 and NL4. Thus, autoinhibition of Collybistin via the $\mathrm{SH} 3$ domain ensures that the synaptic features of Collybistin are not effective ectopically at extrasynaptic sites. Additionally, the inability of NL1 and NL3 to bind or activate Collybistin explains why these Neuroligin isoforms lack the signaling properties to initiate inhibitory synaptic assembly.

This model holds true for the assembly of perisomatic inhibitory synapses, since deletion of both NL2 and Collybistin leads to deficits in somatic Gephyrin and $\mathrm{GABA}_{\mathrm{A}}$ receptor clustering (Papadopoulos et al., 2007; Poulopoulos et al. 2009). Further investigation is needed to determine whether a similar mechanism underlies the assembly of inhibitory synapses on dendrites. The fact that the Gephyrin and consequentially, of $\mathrm{GABA}_{\mathrm{A}}$ receptor at dendritic synapses is affected in the Collybistin KO, but not in the NL2 KO, indicates that NL2independent mechanisms may compensate for or participate in the assembly of 
these inhibitory synapses. Such an alternative mechanism may involve NL4, another Neuroligin paralogue that is capable of activating Collybistin (see below). Additionally, the $\mathrm{GABA}_{\mathrm{A}}$ receptor $\alpha 2$ subunit was shown to bind to and activate Collybistin in cell based assays, leading to the generation of submembrane Gephyrin clusters upon coexpression with $\mathrm{CB}_{\mathrm{SH}_{3+}}$ (Saiepour et al., 2010). Although these findings are preliminary and in conflict with the general understanding of the concept of synaptic assembly - which assumes that specific localization of the scaffolding molecules determine the recruitment of neurotransmitter receptors - it provides hints towards the existence of additional mechanisms for the assembly of the Gephyrin scaffold that do not involve NL2 mediated Collybistin activation. Whether these alternative mechanisms are functioning constitutively in vivo, or whether they are merely switched on in the absence of NL2 remains to be clarified.

In contrast to NL2, deletion of Collybistin leads to severe deficits in both perisomatic and dendritic inhibition in the hippocampus (Papadopoulos et al., 2007; Jedlicka et al., 2009). However, unlike NL2 KO, Collybistin KO mice display no alteration of the membrane recruitment of Gephyrin and GlyRs in the brainstem. Additionally, these mice exhibit severe impairments in GABAergic but not in glycinergic transmission (Papadapoulos et al., 2007). Thus, it appears that, in contrast to somata specific effect of NL2 KO, deletion of Collybistin leads to tissue specific effects, giving rise to deficits in Gephyrin and $G A B A_{A} R$ clustering in the hippocampus and amygdala, but displaying no effects on Gephyrin or Gephyrin-associated GlyR clustering in the brainstem. A more detailed analysis of Collybistin function will be required to determine whether the loss of Collybistin in areas like the brainstem and the spinal cord is compensated by alternative molecules or whether Collybistin is simply not involved in synaptic clustering of Gephyrin in these areas. A recent study using a novel polyclonal Collybistin antibody raised against $\mathrm{CB}_{\mathrm{SH}_{3}+}$ has shown that this isoform of Collybistin specifically colocalizes with $\alpha 2$ subunit containing $\mathrm{GABA}_{\mathrm{A}}$ receptors, but not with GlyRs in the retina (Sailepour et al., 2010), where the glycinergic and GABAergic synapses are largely separated (Wässle et 
al., 2009). These observations are in line with the assumption that Collybistin is not required for Gephyrin-dependent GlyR clustering. However, in contrast, it was also shown in the present study that Collybistin is coimmunoprecipitated with NL2 and NL4 after crosslinking the mixed brainstem and spinal cord postnuclear homogenates, indicating that in these regions Collybistin can assemble into higher order complexes with NL2, NL4 and Gephyrin. Hence, Collybistin might be present at synaptic sites in the brainstem and spinal cord; nevertheless it might be redundant for Gephyrin clustering and may perform other -yet unknown- tasks at the postsynaptic membrane. A detailed immunohistochemical study using a specific antibody is expected to solve the enigma about the region-specific functions of Collybistin.

\subsubsection{The role of NL4 at the inhibitory synapses}

The fact that NL2 specifically interacts with the SH3 domain of Collybistin is in line with the previous observations that NL2, but not NL1 or NL3, has the capacity to activate Collybistin (Poulopoulos et al., 2009). The role of NL4 in regulating the assembly of excitatory or inhibitory synapses had so far remained elusive. We have recently identified NL4 at Gephyrin-positive inhibitory synapses at least in some parts of the CNS (Hoon et al., 2011), and I show here by $\mathrm{Y} 2 \mathrm{H}$ and GST-pulldown assays that NL4 also has the capacity to bind Collybistin (Figure 1B-C), probably in a mode analogous to the NL2-SH3 domain interaction. As in the NL2-Collybistin interaction, the G62A mutation in the SH3 domain of Collybistin leads to loss of interaction with NL4 (Figure 3).

Since NL4 shares the ability with NL2 to interact with Collybistin, it is reasonable to speculate that NL4, as NL2, has the capacity to activate Collybistin. Due to the absence of a NL4 expression plasmid which yields high expression of NL4 in fibroblast cell lines and the deficits in surface trafficking of NL4 in these cells (not shown), chimeric Neuroligin constructs were cloned and tested in cell based Collybistin activation assays. Neither HA-NL3, nor a chimeric HA-NL2ecd-NL1icd protein, which is composed of extracellular and 
transmembrane/intracytoplasmic domains of NL2 and NL1, respectively, were able to activate Collybistin and induce submembrane recruitment of Gephyrin/Collybistin complex, parallel to what has been observed previously with myc-NL1 and HA-NL3 constructs (Poulopoulos et al., 2009). Interestingly, another chimeric Neuroligin construct, $\mathrm{HA}-\mathrm{NL} 2_{\mathrm{ECD}}-\mathrm{NL} 4_{\mathrm{ICD}}$ made up of extracellular and transmembrane/intracytoplasmic domains of NL2 and NL4, respectively, can activate Collybistin and leads to formation of submembrane microaggregates of Gephyrin, to the same extent as HA-NL2, which has been reported as the first NL2 paralogue to act on Collybistin (Figure 2). Based on these observations, I can state that the ICDs of NL2 and NL4 are both capable of mediating a signal that leads to membrane recruitment of Gephyrin/Collybistin complexes. Since replacing the ICD of NL2 with that of NL1 led to a nonfunctional chimeric construct, I assume that the extracellular domain of NL2 does not contribute directly to the role of NL2 in Collybistin activation. Thus, the ICD of NL4 meets the requirements of a functional module that can initiate events leading to inhibitory postsynapse assembly.

The capacity of NL4 to bind to and activate Collybistin defines it as a promising candidate for compensating for the loss of NL2 at the dendritic synapses. In fact, in the retina of NL2 KO mice, a clear upregulation of NL4 has been observed (Hoon et al., 2011), indicating that NL4 may replace NL2 at a subset of synapses throughout the brain and that these two Neuroligin paralogues are functionally related. In the hippocampus, the association of NL4 with excitatory versus inhibitory synapses remains to be identified. A detailed investigation of Gephyrin and $\mathrm{GABA}_{\mathrm{A}} \mathrm{R}$ clustering in the hippocampus of NL4 KO and NL2-4 double KO mice may provide valuable data regarding the role of $\mathrm{NL} 4$ at GABAergic inhibitory synapses. Furthermore, the observation that NL4 forms in vivo complexes with Gephyrin and Collybistin in spinal cord and brainstem is in contradiction with the mouse genetic studies, which postulated a functional redundancy of Collybistin at the glycinergic synapses (Papadopoulos et al., 2007). In order to reconcile these diverging observations, a detailed 
investigation of inhibitory synapse assembly of glycinergic synapses of the brainstem and spinal cord would be essential.

\subsection{Regulated Association of Neuroligins with Scaffolding Molecules}

Due to the fact that all Neuroligins contain highly conserved PDZ- and Gephyrin-binding motifs, Neuroligins, in principle, could associate promiscuously with the scaffolds of excitatory and inhibitory postsynapses. However, the current evidence indicates that throughout the CNS, NLs have differential patterns of association with excitatory and inhibitory synapse specific scaffolding proteins (§ 1.2.1). Therefore, a molecular mechanism that regulates the association of NLs with the scaffolding molecules may exist in vivo. Here, I propose that such a mechanism may involve phosphorylation of a serine residue located within the Gephyrin-binding motifs of NL1, -3 and -4 , the Neuroligin paralogues that are not constitutively localized to inhibitory synapses. We have found that NL3 is phosphorylated at S799 (Box 1). Furthermore, we have observed that in heterologous cells, a point mutation of NL3 that mimicks this serine phosphorylation (S799D) causes deficits in coclustering of NL3 with Gephyrin and $\mathrm{CB}_{\mathrm{SH}_{3} \text {-, }}$ while a phosphorylation deficient mutant (S799A) of NL3 does not seem to obstruct association with Gephyrin microaggregates (Figure 12). These data indicate that phosphorylation of the Gephyrin binding motifs of NL1, NL3 and NL4 may regulate the association of these NLs with Gephyrin in vivo, while NL2 is capable of escaping from such a regulatory mechanisms due to the absence of the serine residue that constitutes the target of phosphorylation.

Since it is preferentially localized to excitatory postsynapses that are comprised of PDZ-domain containing scaffolds and mostly absent from Gephyrin-positive postsynapses, I would expect NL1 to be constitutively phosphorylated. In fact, in neuron cultures, NMDA receptor mediated activation of CaMKII was shown 
to mediate the synapse-boosting activity of NL1 (Chubykin et al., 2007). CaMKII may be responsible for phosphorylating NL1 and thereby restricting it to excitatory postsynapses. The other Neuroligin paralogues, NL3 and NL4, appear to be partitioned to excitatory and inhibitory synapses in different parts of the CNS. NL3 seems to be localized to excitatory synapses in the hippocampus, cerebral cortex and cerebellum, whereas in the brainstem and striatum it is mostly localized to inhibitory synapses (Annarita Patrizi, Torino; personal communication). NL4, on the other hand, is localized to inhibitory synapses in the forebrain, thalamus, brainstem and spinal cord and is specifically localized to excitatory synapses in the barrel cortex (Hoon et al., 2011; Matthieu Hammer; personal communication). The phosphorylation-based mechanism that I propose may underlie the differential distribution of NL3 and NL4 to PSD95 or Gephyrin-positive postsynapses in different brain regions.

A phosphospecific antibody raised against the phoshphorylated peptide that encompasses the 15-residue long Gephyrin binding motif of NL1, NL3 and NL4 is a useful tool to study the tissue-specific phosphorylation of Neuroligins in various parts of the brain. Using this antibody, it was possible to confirm that NL3 is phosphorylated in the brain. In the future, by comparing the phosphorylation levels of specific Neuroligin paralogues in different brain tissues, I am planning to test whether phosphorylation levels of individual Neuroligins in different brain regions display any correlation to their degree of preference for excitatory postsynapses. 


\section{SUMMARY and CONCLUSIONS}

Synaptic inhibition is important for shaping neuronal communication and balancing the activity of brain networks. Understanding the architecture and the mechanisms underlying the formation of inhibitory synapses is essential for unraveling the mysteries about the brain function and to shedding light onto some of the neurological disease conditions. Neuroligin 2 (NL2), a synaptic cell adhesion molecule, is a central organizer of inhibitory postsynapses. NL2 accumulates across GABAergic or glycinergic presynaptic terminals to establish nucleation sites that demarcate the deployment of the Gephyrin scaffold at the plasma membrane, which in turn leads to recruitment and stabilization of GABA and glycine receptors at the postsynaptic sites. NL2 is able to activate Collybistin, an adaptor molecule that links Gephyrin to the plasma membrane. This work has focused on identifying the molecular mechanisms underlying the NL2 function for driving the inhibitory postsynaptic differentiation and investigating the potential roles of the other Neuroligin paralogues at inhibitory postsynapses.

Evidence presented in this study indicates that the $\mathrm{N}$-terminal $\mathrm{SH} 3$ domain of Collybistin inhibits the membrane recruitment of this protein via an intramolecular interaction, which hinders binding of its $\mathrm{PH}$ domain to a specific membrane phosphoinositide. NL2, but not NL1 or NL3, binds directy to the $\mathrm{SH} 3$ domain of Collybistin and alleviates the SH3 domain-mediated inhibition by inducing a conformational change towards a more open state in Collybistin structure. This NL2-induced allosteric rearrangement in Collybistin structure leads to membrane tethering of the Collybistin/Gephyrin complex, which then allows for postsynaptic recruitment and stabilization of inhibitory neurotransmitter receptors. Further investigation of Neuroligin function at the inhibitory synapses indicated that NL4 is yet another Neuroligin isoform that binds to and activates Collybistin and mediates membrane clustering of Gephyrin, suggesting that NL4 may also function as an organizer of inhibitory 
postsynapses. Finally, an additional mechanism is proposed which involves phosphorylation of Gephyrin-binding motifs of the Neuroligin paralogues that are not strictly confined to inhibitory synapses. According to this model, a serine-phosphorylation in the ICD of NL3 may hamper its association with the Gephyrin scaffold. By regulating the association of Neuroligin paralogues with different types of postsynaptic scaffolding molecules, this phosphorylation-based mechanism may underlie the diverse roles of Neuroligin paralogues at excitatory and inhibitory synapses.

This study describes how the tripartite complex formed between an adhesion (NL2), a scaffold (Gephyrin) and an adaptor (Collybistin) protein drives the inhibitory postsynaptic differentiation. The molecular models presented in this study will help formulating a coherent model for the assembly of inhibitory synapses and development of synaptic inhibition in the mammalian CNS. 


\section{BIBLIOGRAPHY}

Aghazadeh, B., Lowry, W.E., Huang, X.Y., and Rosen, M.K. (2000). Structural basis for relief of autoinhibition of the Dbl homology domain of protooncogene Vav by tyrosine phosphorylation. Cell 102, 625-633.

Ago, T., Kuribayashi, F., Hiroaki, H., Takeya, R., Ito, T., Kohda, D., and Sumimoto, H. (2003). Phosphorylation of p47phox directs phox homology domain from $\mathrm{SH} 3$ domain toward phosphoinositides, leading to phagocyte NADPH oxidase activation. Proc Natl Acad Sci U S A 100, 4474-79.

Ahmad, K.F., and Lim, W.A. (2010). The minimal autoinhibited unit of the guanine nucleotide exchange factor intersectin. PLoS One 5, e11291.

Andreotti, A.H., Bunnell, S.C., Feng, S., Berg, L.J., and Schreiber, S.L. (1997). Regulatory intramolecular association in a tyrosine kinase of the Tec family. Nature 385, 93-97.

Aoto, J., Ting, P., Maghsoodi, B., Xu, N., Henkemeyer, M., \& Chen, L. (2007). Postsynaptic ephrinb3 promotes shaft glutamatergic synapse formation. The Journal of Neuroscience : The Official Journal of the Society for Neuroscience, 27(28), 7508-19.

Baer, K., Waldvogel, H.J., Faull, R.L., and Rees, M.I. (2009). Localization of glycine receptors in the human forebrain, brainstem, and cervical spinal cord: an immunohistochemical review. Front Mol Neurosci 2, 25.

Barilá, D., and Superti-Furga, G. (1998). An intramolecular SH3-domain interaction regulates c-Abl activity. Nat Genet 18, 280-82.

Baumeister, M.A., Rossman, K.L., Sondek, J., and Lemmon, M.A. (2006). The Dbs $\mathrm{PH}$ domain contributes independently to membrane targeting and regulation of guanine nucleotide-exchange activity. Biochem J 400, 563 572 .

Bennett, M. V. (1997). Gap junctions as electrical synapses. Journal of Neurocytology, 26(6), 349-66.

Betz, H., Kuhse, J., Schmieden, V., Malosio, M.L., Langosch, D., Prior, P., Schmitt, B., and Kirsch, J. (1991). How to build a glycinergic postsynaptic membrane. J Cell Sci Suppl 15, 23-25.

Betz, A., Okamoto, M., Benseler, F., and Brose, N. (1997). Direct interaction of the rat unc-13 homologue Munc13-1 with the $N$ terminus of syntaxin. J Biol Chem 272, 2520-26. 
Biederer, T., Sara, Y., Mozhayeva, M., Atasoy, D., Liu, X., Kavalali, E.T., and Südhof, T.C. (2002). SynCAM, a synaptic adhesion molecule that drives synapse assembly. Science 297, 1525-531.

Boeckers, T. M. (2006). The postsynaptic density. Cell and Tissue Research, 326(2), 409-22.

Bolliger, M.F., Frei, K., Winterhalter, K.H., and Gloor, S.M. (2001). Identification of a novel neuroligin in humans which binds to PSD-95 and has a widespread expression. Biochem J 356, 581-88.

Bormann, J. (2000). The 'ABC' of GABA receptors. Trends Pharmacol Sci 21, 16-19.

Boucard, A.A., Chubykin, A.A., Comoletti, D., Taylor, P., and Südhof, T.C. (2005). A splice code for trans-synaptic cell adhesion mediated by binding of neuroligin 1 to alpha- and beta-neurexins. Neuron 48, 229-236.

Brenner, R.P. (2004). EEG in convulsive and nonconvulsive status epilepticus. J Clin Neurophysiol 21, 319-331.

Budreck, E.C., and Scheiffele, P. (2007). Neuroligin-3 is a neuronal adhesion protein at GABAergic and glutamatergic synapses. Eur J Neurosci 26, 1738748.

Bustelo, X.R., Sauzeau, V., and Berenjeno, I.M. (2007). GTP-binding proteins of the Rho/Rac family: regulation, effectors and functions in vivo. Bioessays 29, 356-370.

Chan, A.M., Takai, S., Yamada, K., and Miki, T. (1996). Isolation of a novel oncogene, NET1, from neuroepithelioma cells by expression cDNA cloning. Oncogene 12, 1259-266.

Chang, Y., Wang, R., Barot, S., and Weiss, D.S. (1996). Stoichiometry of a recombinant GABAA receptor. J Neurosci 16, 5415-424.

Chen, L., Chetkovich, D.M., Petralia, R.S., Sweeney, N.T., Kawasaki, Y., Wenthold, R.J., Bredt, D.S., and Nicoll, R.A. (2000). Stargazin regulates synaptic targeting of AMPA receptors by two distinct mechanisms. Nature 408, 936-943.

Chih, B., Gollan, L., and Scheiffele, P. (2006). Alternative splicing controls selective trans-synaptic interactions of the neuroligin-neurexin complex. Neuron 51, 171-78.

Chilton, J.K. (2006). Molecular mechanisms of axon guidance. Dev Biol 292, 13-24. 
Chubykin, A.A., Atasoy, D., Etherton, M.R., Brose, N., Kavalali, E.T., Gibson, J.R., and Südhof, T.C. (2007). Activity-dependent validation of excitatory versus inhibitory synapses by neuroligin-1 versus neuroligin-2. Neuron 54, 919-931.

Cline, H. (2005). Synaptogenesis: a balancing act between excitation and inhibition. Curr Biol 15, R203-05.

Craig, A.M., Graf, E.R., and Linhoff, M.W. (2006). How to build a central synapse: clues from cell culture. Trends Neurosci 29, 8-20.

Danglot, L., Triller, A., and Bessis, A. (2003). Association of Gephyrin with synaptic and extrasynaptic GABAA receptors varies during development in cultured hippocampal neurons. Mol Cell Neurosci 23, 264-278.

de Wit, J., Sylwestrak, E., O'Sullivan, M.L., Otto, S., Tiglio, K., Savas, J.N., Yates, J.R., Comoletti, D., Taylor, P., and Ghosh, A. (2009). LRRTM2 interacts with Neurexin1 and regulates excitatory synapse formation. Neuron 64, 799-806.

DiNitto, J.P., and Lambright, D.G. (2006). Membrane and juxtamembrane targeting by PH and PTB domains. Biochim Biophys Acta 1761, 850-867.

Essrich, C., Lorez, M., Benson, J.A., Fritschy, J.M., and Lüscher, B. (1998). Postsynaptic clustering of major GABAA receptor subtypes requires the gamma 2 subunit and Gephyrin. Nat Neurosci 1, 563-571.

Falasca, M., Hughes, W.E., Dominguez, V., Sala, G., Fostira, F., Fang, M.Q., Cazzolli, R., Shepherd, P.R., James, D.E., and Maffucci, T. (2007). The role of phosphoinositide 3-kinase C2alpha in insulin signaling. J Biol Chem 282, 28226-236.

Falasca, M., and Maffucci, T. (2006). Emerging roles of phosphatidylinositol 3monophosphate as a dynamic lipid second messenger. Arch Physiol Biochem 112, 274-284.

Falasca, M., and Maffucci, T. (2009). Rethinking phosphatidylinositol 3monophosphate. Biochim Biophys Acta 1793, 1795-1803.

Feng, G., Tintrup, H., Kirsch, J., Nichol, M.C., Kuhse, J., Betz, H., and Sanes, J.R. (1998). Dual requirement for Gephyrin in glycine receptor clustering and molybdoenzyme activity. Science 282, 1321-24.

Fritschy, J.M., Johnson, D.K., Mohler, H., and Rudolph, U. (1998). Independent assembly and subcellular targeting of GABA(A)-receptor subtypes demonstrated in mouse hippocampal and olfactory neurons in vivo. Neurosci Lett 249, 99-102. 
Fu, Y., and Huang, Z.J. (2010). Differential dynamics and activity-dependent regulation of alpha- and beta-neurexins at developing GABAergic synapses. Proc Natl Acad Sci USA 107, 22699-2704.

Fuhrmann, J.C., Kins, S., Rostaing, P., El Far, O., Kirsch, J., Sheng, M., Triller, A., Betz, H., and Kneussel, M. (2002). Gephyrin interacts with Dynein light chains 1 and 2, components of motor protein complexes. J Neurosci 22, 5393-5402.

Gasnier, B. (2000). The loading of neurotransmitters into synaptic vesicles. Biochimie 82, 327-337.

Gerstner, W., Kreiter, A.K., Markram, H., and Herz, A.V. (1997). Neural codes: firing rates and beyond. Proc Natl Acad Sci U S A 94, 12740-41.

Giesemann, T., Schwarz, G., Nawrotzki, R., Berhörster, K., Rothkegel, M., Schlüter, K., Schrader, N., Schindelin, H., Mendel, R.R., Kirsch, J. \& Jockusch, B.M. (2003). Complex formation between the postsynaptic scaffolding protein gephyrin, profilin, and Mena: a possible link to the microfilament system. J Neurosci 23, 8330-39.

Gillooly, D.J., Simonsen, A., and Stenmark, H. (2001). Cellular functions of phosphatidylinositol 3-phosphate and FYVE domain proteins. Biochem J $355,249-258$.

Graf, E. R., Zhang, X., Jin, S. -X., Linhoff, M. W., \& Craig, A. M. (2004). Neurexins induce differentiation of GABA and glutamate postsynaptic specializations via neuroligins. Cell, 119(7), 1013-26.

Graf, E.R., Kang, Y., Hauner, A.M., and Craig, A.M. (2006). Structure function and splice site analysis of the synaptogenic activity of the neurexin-1 beta LNS domain. J Neurosci 26, 4256-265.

Grosskreutz, Y., Hermann, A., Kins, S., Fuhrmann, J.C., Betz, H., and Kneussel, M. (2001). Identification of a Gephyrin-binding motif in the GDP/GTP exchange factor Collybistin. Biol Chem 382, 1455-462.

Grudzinska, J., Schemm, R., Haeger, S., Nicke, A., Schmalzing, G., Betz, H., and Laube, B. (2005). The beta subunit determines the ligand binding properties of synaptic glycine receptors. Neuron 45, 727-739.

Hanus, C., Vannier, C., and Triller, A. (2004). Intracellular association of glycine receptor with Gephyrin increases its plasma membrane accumulation rate. J Neurosci 24, 1119-128.

Harvey, K., Duguid, I.C., Alldred, M.J., Beatty, S.E., Ward, H., Keep, N.H., 
Lingenfelter, S.E., Pearce, B.R., Lundgren, J., Owen, M.J., Smart, T.G., Lüscher, B., Rees, M.I., Harvey, R.J. (2004). The GDP-GTP exchange factor Collybistin: an essential determinant of neuronal Gephyrin clustering. J Neurosci 24, 5816-826.

Haslam RJ, Koide HB, Hemmings BA. (1993). Pleckstrin domain homology. Nature 363, 309-310.

Hirao, K., Hata, Y., Ide, N., Takeuchi, M., Irie, M., Yao, I., Deguchi, M., Toyoda, A., Sudhof, T.C., and Takai, Y. (1998). A novel multiple PDZ domain-containing molecule interacting with N-methyl-D-aspartate receptors and neuronal cell adhesion proteins. J Biol Chem 273, 21105-110.

Hoon, M., Bauer, G., Fritschy, J.-M., Moser, T., Falkenburger, B.H., and Varoqueaux, F. (2009). Neuroligin 2 controls the maturation of GABAergic synapses and information processing in the retina. J Neurosci 29, 8039-050.

Hoon, M., Soykan, T., Falkenburger, B., Hammer, M., Patrizi, A., Schmidt, K.-F., Sassoè-Pognetto, M., Löwel, S., Moser, T., Taschenberger, H., Brose, N. \& Varoqueaux, F. (2011). Neuroligin-4 is localized to glycinergic postsynapses and regulates inhibition in the retina. Proc Natl Acad Sci USA. 108, 3053-8

Hyvönen, M., Macias, M.J., Nilges, M., Oschkinat, H., Saraste, M., and Wilmanns, M. (1995). Structure of the binding site for inositol phosphates in a PH domain. EMBO J 14, 4676-685.

Ichtchenko, K., Hata, Y., Nguyen, T., Ullrich, B., Missler, M., Moomaw, C., and Südhof, T.C. (1995). Neuroligin 1: a splice site-specific ligand for betaneurexins. Cell 81, 435-443.

Ichtchenko, K., Nguyen, T., and Südhof, T.C. (1996). Structures, alternative splicing, and neurexin binding of multiple neuroligins. J Biol Chem 271, 2676-682.

lida, J., Hirabayashi, S., Sato, Y., and Hata, Y. (2004). Synaptic scaffolding molecule is involved in the synaptic clustering of neuroligin. Mol Cell Neurosci 27, 497-508.

Irie, M., Hata, Y., Takeuchi, M., Ichtchenko, K., Toyoda, A., Hirao, K., Takai, Y., Rosahl, T.W., and Südhof, T.C. (1997). Binding of neuroligins to PSD-95. Science 277, 1511-15.

Jamain, S., Radyushkin, K., Hammerschmidt, K., Granon, S., Boretius, S., Varoqueaux, F., Ramanantsoa, N., Gallego, J., Ronnenberg, A., Winter, D., Frahm, J., Fischer, J., Bourgeron, T., Ehrenreich, H. \& Brose, N. (2008). Reduced social interaction and ultrasonic communication in a mouse model of monogenic heritable autism. Proc Natl Acad Sci USA 105, 1710-15. 
Jedlicka, P., Papadopoulos, T., Deller, T., Betz, H., and Schwarzacher, S.W. (2009). Increased network excitability and impaired induction of long-term potentiation in the dentate gyrus of Collybistin-deficient mice in vivo. Mol Cell Neurosci 41, 94-100.

Jedlicka, P., Hoon, M., Papadopoulos, T., Vlachos, A., Winkels, R., Poulopoulos, A., Betz, H., Deller, T., Brose, N., Varoqueaux, F. \& Schwarzacher, S.W. (2011). Increased dentate gyrus excitability in neuroligin-2-deficient mice in vivo. Cereb Cortex 21, 357-367.

Jiang, M., and Chen, G. (2006). High Ca2+-phosphate transfection efficiency in low-density neuronal cultures. Nat Protoc 1, 695-700.

Kalscheuer, V.M., Musante, L., Fang, C., Hoffmann, K., Fuchs, C., Carta, E., Deas, E., Venkateswarlu, K., Menzel, C., Ullmann, R., Tommerup, N., Dalprà, L., Tzschach, A., Selicorni, A., Lüscher, B., Ropers, H.-H., Harvey, K. \& Harvey, R.J. (2009). A balanced chromosomal translocation disrupting ARHGEF9 is associated with epilepsy, anxiety, aggression, and mental retardation. Hum Mutat 30, 61-68.

Kang, Y., Zhang, X., Dobie, F., Wu, H., and Craig, A.M. (2008). Induction of GABAergic postsynaptic differentiation by alpha-neurexins. J Biol Chem 283, 2323-334.

Kawasaki, Y., Senda, T., Ishidate, T., Koyama, R., Morishita, T., Iwayama, Y., Higuchi, O., and Akiyama, T. (2000). Asef, a link between the tumor suppressor APC and G-protein signaling. Science 289, 1194-97.

Kayser, M. S., McClelland, A. C., Hughes, E. G., \& Dalva, M. B. (2006). Intracellular and trans-synaptic regulation of glutamatergic synaptogenesis by ephb receptors. The Journal of Neuroscience : The Official Journal of the Society for Neuroscience, 26(47), 12152-64.

Kayser, M.S., Nolt, M.J., and Dalva, M.B. (2008). EphB receptors couple dendritic filopodia motility to synapse formation. Neuron 59, 56-69.

Kim, S., Burette, A., Chung, H.S., Kwon, S.-K., Woo, J., Lee, H.W., Kim, K., Kim, H., Weinberg, R.J., and Kim, E. (2006). NGL family PSD-95-interacting adhesion molecules regulate excitatory synapse formation. Nat Neurosci 9, 1294-1301.

Kins, S., Kuhse, J., Laube, B., Betz, H., and Kirsch, J. (1999). Incorporation of a Gephyrin-binding motif targets NMDA receptors to Gephyrin-rich domains in HEK 293 cells. Eur J Neurosci 11, 740-44. 
Kins, S., Betz, H., and Kirsch, J. (2000). Collybistin, a newly identified brainspecific GEF, induces submembrane clustering of Gephyrin. Nat Neurosci 3, 22-29.

Kirsch, J., Wolters, I., Triller, A., and Betz, H. (1993a). Gephyrin antisense oligonucleotides prevent glycine receptor clustering in spinal neurons. Nature 366, 745-48.

Kirsch, J., Malosio, M.L., Wolters, I., and Betz, H. (1993b). Distribution of Gephyrin transcripts in the adult and developing rat brain. Eur J Neurosci 5, 1109-117.

Kirsch, J., Kuhse, J., and Betz, H. (1995). Targeting of glycine receptor subunits to Gephyrin-rich domains in transfected human embryonic kidney cells. Mol Cell Neurosci 6, 450-461.

Kneussel, M., Hermann, A., Kirsch, J., and Betz, H. (1999a). Hydrophobic interactions mediate binding of the glycine receptor beta-subunit to Gephyrin. J Neurochem 72, 1323-26.

Kneussel, M., Brandstätter, J.H., Laube, B., Stahl, S., Müller, U., and Betz, H. (1999b). Loss of postsynaptic $\operatorname{GABA}(\mathrm{A})$ receptor clustering in Gephyrindeficient mice. J Neurosci 19, 9289-297.

Kneussel, M., and Betz, H. (2000). Clustering of inhibitory neurotransmitter receptors at developing postsynaptic sites: the membrane activation model. Trends Neurosci 23, 429-435.

Kneussel, M., Brandstätter, J.H., Gasnier, B., Feng, G., Sanes, J.R., and Betz, H. (2001). Gephyrin-independent clustering of postsynaptic GABA(A) receptor subtypes. Mol Cell Neurosci 17, 973-982.

Ko, J., Fuccillo, M.V., Malenka, R.C., and Südhof, T.C. (2009). LRRTM2 functions as a neurexin ligand in promoting excitatory synapse formation. Neuron 64, 791-98.

Koehnke, J., Katsamba, P.S., Ahlsen, G., Bahna, F., Vendome, J., Honig, B., Shapiro, L., and Jin, X. (2010). Splice form dependence of betaneurexin/neuroligin binding interactions. Neuron 67, 61-74.

Kornau, H.C., Schenker, L.T., Kennedy, M.B., and Seeburg, P.H. (1995). Domain interaction between NMDA receptor subunits and the postsynaptic density protein PSD-95. Science 269, 1737-740.

Laemmli, U.K. (1970). Cleavage of structural proteins during the assembly of the head of bacteriophage T4. Nature 227, 680-85. 
Langosch, D., Thomas, L., and Betz, H. (1988). Conserved quaternary structure of ligand-gated ion channels: the postsynaptic glycine receptor is a pentamer. Proc Natl Acad Sci U S A 85, 7394-98.

Lemmon, M.A., Ferguson, K.M., O'Brien, R., Sigler, P.B., and Schlessinger, J. (1995). Specific and high-affinity binding of inositol phosphates to an isolated pleckstrin homology domain. Proc Natl Acad Sci U S A 92, 1047276.

Lerner, E.C., and Smithgall, T.E. (2002). SH3-dependent stimulation of Srcfamily kinase autophosphorylation without tail release from the $\mathrm{SH} 2$ domain in vivo. Nat Struct Biol 9, 365-69.

Lévi, S., Logan, S.M., Tovar, K.R., and Craig, A.M. (2004). Gephyrin is critical for glycine receptor clustering but not for the formation of functional GABAergic synapses in hippocampal neurons. J Neurosci 24, 207-217.

Levinson, J.N., and El-Husseini, A. (2005). Building excitatory and inhibitory synapses: balancing neuroligin partnerships. Neuron 48, 171-74.

Lim, W.A., Richards, F.M., and Fox, R.O. (1994). Structural determinants of peptide-binding orientation and of sequence specificity in $\mathrm{SH} 3$ domains. Nature 372, 375-79.

Linhoff, M.W., Laurén, J., Cassidy, R.M., Dobie, F.A., Takahashi, H., Nygaard, H.B., Airaksinen, M.S., Strittmatter, S.M., and Craig, A.M. (2009). An unbiased expression screen for synaptogenic proteins identifies the LRRTM protein family as synaptic organizers. Neuron 61, 734-749.

Lüscher, B., and Keller, C.A. (2004). Regulation of GABAA receptor trafficking, channel activity, and functional plasticity of inhibitory synapses. Pharmacol Ther 102, 195-221.

Lynch, J. W. (2004). Molecular structure and function of the glycine receptor chloride channel. Physiological reviews, 84(4), 1051-1095.

Maas, C., Tagnaouti, N., Loebrich, S., Behrend, B., Lappe-Siefke, C., and Kneussel, M. (2006). Neuronal cotransport of glycine receptor and the scaffold protein Gephyrin. J Cell Biol 172, 441-451.

Maas, C., Belgardt, D., Lee, H.K., Heisler, F.F., Lappe-Siefke, C., Magiera, M.M., van Dijk, J., Hausrat, T.J., Janke, C., and Kneussel, M. (2009). Synaptic activation modifies microtubules underlying transport of postsynaptic cargo. Proc Natl Acad Sci USA 106, 8731-36.

Macias, M.J., Wiesner, S., and Sudol, M. (2002). WW and SH3 domains, two different scaffolds to recognize proline-rich ligands. FEBS Lett 513, 30-37. 
Maffucci, T., Brancaccio, A., Piccolo, E., Stein, R.C., and Falasca, M. (2003). Insulin induces phosphatidylinositol-3-phosphate formation through TC10 activation. EMBO J 22, 4178-189.

Magee, J. (2000). Dendritic integration of excitatory synaptic input. Nature reviews Neuroscience. 1, 181-190.

Mayer, B.J., Saksela, K. (2005). SH3 domains. In Cesareni, G., Gimona, M., Sudol, M. \& Yaffe,M. (Eds.) Modular Protein Domains. (pp 37-58). WileyVCH Verlag $\mathrm{GmbH} \& \mathrm{Co}$. KGaA, Weinheim.

Meier, J., Vannier, C., Sergé, A., Triller, A., and Choquet, D. (2001). Fast and reversible trapping of surface glycine receptors by Gephyrin. Nat Neurosci 4, 253-260.

Meier, J., and Grantyn, R. (2004). A Gephyrin-related mechanism restraining glycine receptor anchoring at GABAergic synapses. J Neurosci 24, 13981405.

Meyer, G., Kirsch, J., Betz, H., and Langosch, D. (1995). Identification of a Gephyrin binding motif on the glycine receptor beta subunit. Neuron 15, 563-572.

Miki, T., Smith, C.L., Long, J.E., Eva, A., and Fleming, T.P. (1993). Oncogene ect2 is related to regulators of small GTP-binding proteins. Nature 362, 46265.

Missler, M., Zhang, W., Rohlmann, A., Kattenstroth, G., Hammer, R.E., Gottmann, K., and Südhof, T.C. (2003). Alpha-neurexins couple Ca2+ channels to synaptic vesicle exocytosis. Nature 423, 939-948.

Mitin, N., Betts, L., Yohe, M.E., Der, C.J., Sondek, J., and Rossman, K.L. (2007). Release of autoinhibition of ASEF by APC leads to CDC42 activation and tumor suppression. Nat Struct Mol Biol 14, 814-823.

Moarefi, I., LaFevre-Bernt, M., Sicheri, F., Huse, M., Lee, C.H., Kuriyan, J., and Miller, W.T. (1997). Activation of the Src-family tyrosine kinase Hck by SH3 domain displacement. Nature 385, 650-53.

Moss, S.J., and Smart, T.G. (2001). Constructing inhibitory synapses. Nat Rev Neurosci 2, 240-250.

Murayama, K., Shirouzu, M., Kawasaki, Y., Kato-Murayama, M., HanawaSuetsugu, K., Sakamoto, A., Katsura, Y., Suenaga, A., Toyama, M., Terada, T., Taiji, M., Akiyama, T. \& Yokoyama, S. (2007). Crystal structure of the rac activator, Asef, reveals its autoinhibitory mechanism. J Biol Chem 282, 
Musacchio, A., Saraste, M., and Wilmanns, M. (1994). High-resolution crystal structures of tyrosine kinase $\mathrm{SH} 3$ domains complexed with proline-rich peptides. Nat Struct Biol 1, 546-551.

Nguyen, T., and Südhof, T.C. (1997). Binding properties of neuroligin 1 and neurexin 1 beta reveal function as heterophilic cell adhesion molecules. J Biol Chem 272, 26032-39.

Nusser, Z., Sieghart, W., Benke, D., Fritschy, J.M., and Somogyi, P. (1996). Differential synaptic localization of two major gamma-aminobutyric acid type A receptor alpha subunits on hippocampal pyramidal cells. Proc Natl Acad Sci U S A 93, 11939-944.

Nusser, Z., Sieghart, W., and Somogyi, P. (1998). Segregation of different GABAA receptors to synaptic and extrasynaptic membranes of cerebellar granule cells. J Neurosci 18, 1693-1703.

Owald, D., and Sigrist, S.J. (2009). Assembling the presynaptic active zone. Curr Opin Neurobiol 19, 311-18.

Paarmann, I., Schmitt, B., Meyer, B., Karas, M., and Betz, H. (2006). Mass spectrometric analysis of glycine receptor-associated Gephyrin splice variants. J Biol Chem 281, 34918-925.

Papadopoulos, T., Eulenburg, V., Reddy-Alla, S., Mansuy, I.M., Li, Y., and Betz, $\mathrm{H}$. (2008). Collybistin is required for both the formation and maintenance of GABAergic postsynapses in the hippocampus. Mol Cell Neurosci 39, 16169.

Papadopoulos, T., Korte, M., Eulenburg, V., Kubota, H., Retiounskaia, M., Harvey, R.J., Harvey, K., O'Sullivan, G.A., Laube, B., Hülsmann, S., Geiger, J.R.P. \& Betz, H. (2007). Impaired GABAergic transmission and altered hippocampal synaptic plasticity in Collybistin-deficient mice. EMBO J 26, 3888-899.

Pirker, S., Schwarzer, C., Wieselthaler, A., Sieghart, W., and Sperk, G. (2000). $\mathrm{GABA}(\mathrm{A})$ receptors: immunocytochemical distribution of 13 subunits in the adult rat brain. Neuroscience 101, 815-850.

Pluk, H., Dorey, K., and Superti-Furga, G. (2002). Autoinhibition of c-Abl. Cell 108, 247-259.

Prange, O., Wong, T.P., Gerrow, K., Wang, Y.T., and El-Husseini, A. (2004). A balance between excitatory and inhibitory synapses is controlled by PSD-95 and neuroligin. Proc Natl Acad Sci USA 101, 13915-920. 
Probst, A., Cortés, R., and Palacios, J.M. (1986). The distribution of glycine receptors in the human brain. A light microscopic autoradiographic study using $[3 \mathrm{H}]$ strychnine. Neuroscience 17, 11-35.

Poulopoulos A. (2008). Mechanisms of neuroligin function in inhibitory postsynaptic differentiation. Ph.D. Thesis. Georg August University Göttingen; Germany

Poulopoulos, A., Aramuni, G., Meyer, G., Soykan, T., Hoon, M., Papadopoulos, T., Zhang, M., Paarmann, I., Fuchs, C., Harvey, K., Jedlicka, P., Schwarzacher, S.W., Betz, H., Harvey, R.J., Brose, N., Zhang, W. \& Varoqueaux, F. (2009). Neuroligin 2 drives postsynaptic assembly at perisomatic inhibitory synapses through Gephyrin and Collybistin. Neuron 63, 628-642.

Pufall, M.A., and Graves, B.J. (2002). Autoinhibitory domains: modular effectors of cellular regulation. Annu Rev Cell Dev Biol 18, 421-462.

Pursglove, S.E., Mulhern, T.D., Mackay, J.P., Hinds, M.G., and Booker, G.W. (2002). The solution structure and intramolecular associations of the Tec kinase SRC homology 3 domain. J Biol Chem 277, 755-762.

Reddy-Alla, S., Schmitt, B., Birkenfeld, J., Eulenburg, V., Dutertre, S., Böhringer, C., Götz, M., Betz, H., and Papadopoulos, T. (2010). PH-domain-driven targeting of Collybistin but not Cdc42 activation is required for synaptic Gephyrin clustering. Eur J Neurosci 31, 1173-184.

Reid, T., Bathoorn, A., Ahmadian, M.R., and Collard, J.G. (1999). Identification and characterization of hPEM-2, a guanine nucleotide exchange factor specific for Cdc42. J Biol Chem 274, 33587-593.

Rizo, J., and Rosenmund, C. (2008). Synaptic vesicle fusion. Nat Struct Mol Biol $15,665-674$.

Rojas, R.J., Yohe, M.E., Gershburg, S., Kawano, T., Kozasa, T., and Sondek, J. (2007). Galphaq directly activates p63RhoGEF and Trio via a conserved extension of the Dbl homology-associated pleckstrin homology domain. J Biol Chem 282, 29201-210.

Ron, D., Graziani, G., Aaronson, S.A., and Eva, A. (1989). The N-terminal region of proto- $\mathrm{dbl}$ down regulates its transforming activity. Oncogene 4, 1067-072.

Rossman, K.L., and Campbell, S.L. (2000). Bacterial expressed DH and DH/PH domains. Methods Enzymol 325, 25-38. 
Saiepour, L., Fuchs, C., Patrizi, A., Sassoè-Pognetto, M., Harvey, R.J., and Harvey, K. (2010). Complex role of Collybistin and Gephyrin in GABAA receptor clustering. J Biol Chem 285, 29623-631.

Saiyed, T., Paarmann, I., Schmitt, B., Haeger, S., Sola, M., Schmalzing, G., Weissenhorn, W., and Betz, H. (2007). Molecular basis of Gephyrin clustering at inhibitory synapses: role of G- and E-domain interactions. J Biol Chem 282, 5625-632.

Sassoè-Pognetto, M., Panzanelli, P., Sieghart, W., and Fritschy, J.M. (2000). Colocalization of multiple $\mathrm{GABA}(\mathrm{A})$ receptor subtypes with Gephyrin at postsynaptic sites. J Comp Neurol 420, 481-498.

Scheiffele, P., Fan, J., Choih, J., Fetter, R., and Serafini, T. (2000). Neuroligin expressed in nonneuronal cells triggers presynaptic development in contacting axons. Cell 101, 657-669.

Schiller, M.R., Chakrabarti, K., King, G.F., Schiller, N.I., Eipper, B.A., and Maciejewski, M.W. (2006). Regulation of RhoGEF activity by intramolecular and intermolecular SH3 domain interactions. J Biol Chem 281, 18774-786.

Schmidt, A., and Hall, A. (2002). Guanine nucleotide exchange factors for Rho GTPases: turning on the switch. Genes Dev 16, 1587-1609.

Schoch, S., \& Gundelfinger, E. D. (2006). Molecular organization of the presynaptic active zone. Cell and tissue research, 326(2), 379-391.

Seabold, G.K., Burette, A., Lim, I.A., Weinberg, R.J., and Hell, J.W. (2003). Interaction of the tyrosine kinase Pyk2 with the N-methyl-D-aspartate receptor complex via the Src homology 3 domains of PSD-95 and SAP102. J Biol Chem 278, 15040-48.

Siddiqui, T.J., Pancaroglu, R., Kang, Y., Rooyakkers, A., and Craig, A.M. (2010). LRRTMs and neuroligins bind neurexins with a differential code to cooperate in glutamate synapse development. J Neurosci 30, 7495-7506.

Sieghart, W., Fuchs, K., Tretter, V., Ebert, V., Jechlinger, M., Höger, H., and Adamiker, D. (1999). Structure and subunit composition of GABA(A) receptors. Neurochem Int 34, 379-385.

Sola, M., Bavro, V.N., Timmins, J., Franz, T., Ricard-Blum, S., Schoehn, G., Ruigrok, R.W.H., Paarmann, I., Saiyed, T., O'Sullivan, G.A., Schmitt, B., Betz, H. \& Weissenhorn, W. (2004). Structural basis of dynamic glycine receptor clustering by Gephyrin. EMBO J 23, 2510-19.

Song, J.Y., Ichtchenko, K., Südhof, T.C., and Brose, N. (1999). Neuroligin 1 is a postsynaptic cell-adhesion molecule of excitatory synapses. Proc Natl Acad 
Sci USA 96, 1100-05.

Sporns, O. (2002) Network analysis, complexity and brain function. Complexity $8,56-60$.

Sterpetti, P., Hack, A.A., Bashar, M.P., Park, B., Cheng, S.D., Knoll, J.H., Urano, T., Feig, L.A., and Toksoz, D. (1999). Activation of the Lbc Rho exchange factor proto-oncogene by truncation of an extended $C$ terminus that regulates transformation and targeting. Mol Cell Biol 19, 1334-345.

Studler, B., Fritschy, J.-M., and Brünig, I. (2002). GABAergic and glutamatergic terminals differentially influence the organization of GABAergic synapses in rat cerebellar granule cells in vitro. Neuroscience 114, 123-133.

Tabuchi, K., and Südhof, T.C. (2002). Structure and evolution of neurexin genes: insight into the mechanism of alternative splicing. Genomics 79, 849-859.

Takeichi, M. (2007). The cadherin superfamily in neuronal connections and interactions. Nat Rev Neurosci 8, 11-20.

Tavares, G.A., Panepucci, E.H., and Brunger, A.T. (2001). Structural characterization of the intramolecular interaction between the $\mathrm{SH} 3$ and guanylate kinase domains of PSD-95. Mol Cell 8, 1313-325.

Towbin, H., Staehelin, T., and Gordon, J. (1979). Electrophoretic transfer of proteins from polyacrylamide gels to nitrocellulose sheets: procedure and some applications. Proc Natl Acad Sci U S A 76, 4350-54.

Tretter, V., Jacob, T.C., Mukherjee, J., Fritschy, J.-M., Pangalos, M.N., and Moss, S.J. (2008). The clustering of $\operatorname{GABA}(A)$ receptor subtypes at inhibitory synapses is facilitated via the direct binding of receptor alpha 2 subunits to Gephyrin. J Neurosci 28, 1356-365.

Triller, A., and Choquet, D. (2003). Synaptic structure and diffusion dynamics of synaptic receptors. Biol Cell 95, 465-476.

van Leeuwen, F.N., van der Kammen, R.A., Habets, G.G., and Collard, J.G. (1995). Oncogenic activity of Tiam1 and Rac1 in NIH3T3 cells. Oncogene $11,2215-221$.

Varoqueaux, F., Jamain, S., and Brose, N. (2004). Neuroligin 2 is exclusively localized to inhibitory synapses. Eur J Cell Biol 83, 449-456.

Varoqueaux, F., Aramuni, G., Rawson, R.L., Mohrmann, R., Missler, M., Gottmann, K., Zhang, W., Südhof, T.C., and Brose, N. (2006). Neuroligins determine synapse maturation and function. Neuron 51, 741-754. 
Vojtek, A.B., Hollenberg, S.M., and Cooper, J.A. (1993). Mammalian Ras interacts directly with the serine/threonine kinase Raf. Cell 74, 205-214.

Wässle, H., Heinze, L., Ivanova, E., Majumdar, S., Weiss, J., Harvey, R.J., and Haverkamp, S. (2009). Glycinergic transmission in the Mammalian retina. Front Mol Neurosci 2, 6 .

Wells, C.D., Gutowski, S., Bollag, G., and Sternweis, P.C. (2001). Identification of potential mechanisms for regulation of p115 RhoGEF through analysis of endogenous and mutant forms of the exchange factor. J Biol Chem 276, 28897-8905.

Wojcik, S. M., \& Brose, N. (2007). Regulation of membrane fusion in synaptic excitation-secretion coupling: speed and accuracy matter. Neuron, 55(1), $11-24$.

Wojcik, S.M., Katsurabayashi, S., Guillemin, I., Friauf, E., Rosenmund, C., Brose, N., and Rhee, J.S. (2006). A shared vesicular carrier allows synaptic corelease of GABA and glycine. Neuron 50, 575-587.

Xiang, S., Kim, E.Y., Connelly, J.J., Nassar, N., Kirsch, J., Winking, J., Schwarz, G., and Schindelin, H. (2006). The crystal structure of Cdc42 in complex with Collybistin II, a Gephyrin-interacting guanine nucleotide exchange factor. J Mol Biol 359, 35-46.

Yamagata, M., Sanes, J.R., and Weiner, J.A. (2003). Synaptic adhesion molecules. Curr Opin Cell Biol 15, 621-632.

Yohe, M.E., Rossman, K., and Sondek, J. (2008). Role of the C-terminal SH3 domain and $\mathrm{N}$-terminal tyrosine phosphorylation in regulation of Tim and related Dbl-family proteins. Biochemistry 47, 6827-839.

Yu, J.W., Mendrola, J.M., Audhya, A., Singh, S., Keleti, D., DeWald, D.B., Murray, D., Emr, S.D., and Lemmon, M.A. (2004). Genome-wide analysis of membrane targeting by $\mathrm{S}$. cerevisiae pleckstrin homology domains. Mol Cell 13, 677-688.

Yu, B., Martins, I.R.S., Li, P., Amarasinghe, G.K., Umetani, J., FernandezZapico, M.E., Billadeau, D.D., Machius, M., Tomchick, D.R., and Rosen, M.K. (2010). Structural and energetic mechanisms of cooperative autoinhibition and activation of Vav1. Cell 140, 246-256.

Yuzawa, S., Ogura, K., Horiuchi, M., Suzuki, N.N., Fujioka, Y., Kataoka, M., Sumimoto, H., and Inagaki, F. (2004). Solution structure of the tandem SrC homology 3 domains of p47phox in an autoinhibited form. J Biol Chem 279, 29752-760. 
Zamanian, J.L., and Kelly, R.B. (2003). Intersectin 1L guanine nucleotide exchange activity is regulated by adjacent src homology 3 domains that are also involved in endocytosis. Mol Biol Cell 14, 1624-637. 


\section{TOLGA SOYKAN}

$\begin{array}{ll}\text { Date of Birth } & \text { October 7th, 1984 } \\ \text { E-mail } & \text { soykan@em.mpg.de } \\ \text { Address } & \text { Nikolaistr 15, 37073 Göttingen, Germany } \\ \text { Telephone } & +49-17663037045\end{array}$

\section{EDUCATIONAL BACKGROUND}

October 2007 - May 2011

PhD, Department of Molecular Neurobiology, Max-Planck Institute of Experimental Medicine, Göttingen, Germany

Thesis title: Neuroligin-2 Induced Allosteric Transition of Collybistin Underlies Inhibitory Postsynaptic Differentiation

Supervisors: Prof. Dr. Nils Brose \& Dr. Frédérique Varoqueaux

October 2006 - May 2011

Georg-August-Universität Göttingen, International Max Planck Research School, MSc/PhD Program in Molecular Biology, Göttingen, Germany

September 2002 - June 2006

BSc, Biological Sciences and Bioengineering, Sabanci University, Istanbul, Turkey

Thesis title: Investigation of metallothionein gene expression in macaroni wheat (Triticum durum) in response to cadmium toxicity

Supervisor: Prof. Dr. Zehra Sayers

September 1995 - June 2002

FMV Isik High School, Istanbul, Turkey

\section{WORK \& TEACHING EXPERIENCE}

\section{September 2010}

Instructor for the Advanced Method Course on "Hippocampal Neurons: Primary Culture and Transfection" within the scope of Göttingen Graduate School of Neurosciences and Biosciences (GGNB), Göttingen, Germany

\section{March 2008 - February 2011}

Teaching assistant for the Cell Adhesion and Signal Transduction Lectures by Prof. Dr. Nils Brose, International Max Planck Research School in Molecular Biology and in Neuroscience, Göttingen, Germany 
July 2005 - September 2005

Summer Internship, Department of Microbiology and Environmental Toxicology, University of California Santa Cruz, CA, USA

Project Title: Investigating the role of nucleotide metabolism associated genes in Vibrio cholerae biofilm formation

Supervisor: Prof. Dr. Fitnat Yildiz

AWARDS \& ACHIEVEMENTS

\section{October 2009}

Best Poster Prize in Synaptic Inhibition in Health and Disease Conference, Chicago, USA

October 2006 - December 2009

International Max Planck Research School Stipend, Göttingen, Germany

October 2002 - June 2006

High Honor Scholarship, Sabanci University, Turkey

June 2002

1 st rank in Chemistry in TUBITAK (Turkish Scientific and Technical Research Foundation) Research Project Competition Among High School Students, Ankara, Turkey

\section{June 2001}

Honorable Mention in International Competition for High School Students in Research Projects in Physics, held by Polish Academy of Sciences, Warsaw, Poland 